\title{
COMPARAÇÃO DE MODELOS MISTOS PARA DADOS DE PROPORÇÕES VISANDO À ESTIMAÇÃO DO COEFICIENTE DE HERDABILIDADE
}

\author{
Telde NATEl Custódio \\ Engenheiro Agrícola
}

Orientador: Prof. Dr. DÉCIO BARBIN

\begin{abstract}
Tese apresentada à Escola Superior de Agricultura "Luiz de Queroz", Universidade de São Paulo, para obtenção do título de Doutor em Agronomia, Área de Concentração: Estatística e Experimentação Agronômica
\end{abstract}

P IR A C I C A B A

Estado de São Paulo - Brasil

Novembro -2004 
TELDE NATEL CUSTÓDIO. Comparação de modelos mistos para dados de proporções visando à estimação do coeficiente de herdabilidade.

p. item linha onde se lê

$5 \quad 2.1 \quad$ nona $u \sim \mathrm{N}(0 ; G)$

$52.1 \quad$ décima $\quad \varepsilon \sim \mathrm{N}(0 ; R)$

$37 \quad 2.5 .2 .1 .1$ décima segunda

$$
W^{*}=\operatorname{diag}\left[a_{i}(\phi) V\left(\mu_{i}\right) \frac{\partial \eta_{i}}{\partial \mu_{i}}\right]
$$

$47 \quad 2.6 \quad$ décima

quarta

$50 \quad 2.7$

$50 \quad 2.7 .1$

segunda

$$
\hat{\sigma}=\frac{\left(Y^{*}-X \hat{\beta}-Z \hat{u}\right) W\left(Y^{*}-X \hat{\beta}-Z \hat{u}\right)}{N-r(x)-q}
$$

é a estimativa da herdabilidade

Componentes da herdabilidade terceira

$51 \quad 2.7 .1$

décima

oitava

$52 \quad 2.7 .2 \quad$ vigésima

53

2.7 .3

décima

sexta

$\begin{array}{ll}55 & 2.7 .3\end{array}$

55

2.7 .3

primeira

envolvendo linhagens

quinta

$\sigma_{e}^{2}+g \sigma_{r l}^{2}$

$80 \quad 4.1 .5$

décima

$h^{2}$ Referência

média dos tratamentos

envolvendo genótipos

envolvendo genótipos

$\sigma_{e}^{2}+g \sigma_{r}^{2} r l$

$h^{2}$ Paramétrico

terceira 
Dados Internacionais de Catalogação na Publicação (CIP) DIVISĀO DE BIBLIOTECA E DOCUMENTAÇÃO - ESALQUSSP

\section{Custódio, Telde Natel}

Comparação de modelos mistos para dados de proporções visando à estimação do coeficiente de herdabilidade / Telde Natel Custódio. - Piracicaba, 2004

$121 \mathrm{p}$.

Tese (Doutorado) - Escola Superior de Agricultura Luiz de Queiroz, 2004.

Bibliografia.

1. Análise de variância 2. Análise multivariada 3. Estatística aplicada 4. Eucalipto 5. Modelo matemático 6. Modelos lineares generalizados 7. Simulação (Estatística)

8. Verossimilhança I. Titulo

CDD 511.8

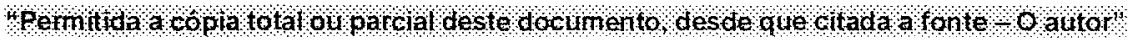


A Nossa Senhora Aparecida, pela infinita bondade e amor, e a meu anjinho da guarda (Lilianein memoriam), que sempre me atendem nos momentos de aflição.

Ao meu querido pai, Lázaro Custódio Neto, e a minha maravilhosa mãe, Julieta da Conceição Custódio, pela forma sábia e dedicada com que nos lançastes na vida, pela coragem, pelo infinito amor, pelos ensinamentos de vida, pela confiança, pelo incentivo $e$ pelo esforço em educar seus filhos.

Aos meus queridos irmãos, Maria Zélia, José Roberto, Elizabeth, Vanderléia, pela união e pelo carinho.

Aos meus queridos sobrinhos, Viviane, Adriano, Guilherme, Vinícius, Fernando, Fabrício e Henrico, pela simpatia e pela alegria que nos proporcionam. 


\section{AGRADECIMENTOS}

A Deus, pela vida e por proporcionar-me a realização desta importante conquista. Ao Prof. Dr. Décio Barbin, pelos ensinamentos, valiosa orientação e amizade.

A Profa. Dra. Clarice Garcia Borges Demétrio, pelos ensinamentos, sugestão do tema, apoio e valiosas sugestões.

A Universidade Federal de Lavras e ao Departamento de Ciências Exatas pelo afastamento para a realização do curso.

A Universidade de São Paulo e a Escola Superior de Agricultura "Luiz de Queiroz", a oportunidade concedida para a realização do curso.

Ao Conselho Nacional de Desenvolvimento Científico e Tecnológico (CNPq) pela bolsa de estudos concedida.

Aos Professores do Departamento de Ciências Exatas da UFLA, Dr. Agostinho Roberto de Abreu, Dr. Joel Augusto Muniz e Dr. Ruben Delly Veiga, o apoio.

Ao Prof. Dr. Cláudio Manoel Rodrigues de Melo (UFSC) pelas valiosas sugestões e amizade.

Aos membros da Banca de Qualificação, Prof. Dr. Roland Vencovsky (ESALQ) e Profa. Dra. Sheila Zambello de Pinho (UNESP) pelas valiosas sugestões.

Aos Professores do curso pelos ensinamentos, em especial ao Prof. Dr. Carlos Tadeu dos Santos Dias, Profa. Dra. Sônia Maria de Stefano Piedade e Prof. Dr. César Gonçalves de Lima.

Ao Pesquisador Dr. Osmir José Lavoranti (EMBRAPA - FLORESTAS), pelos dados de progênies de Eucalipto.

Aos Funcionários técnico-administrativos do Departamento de Ciências Exatas da ESALQ, em especial a Solange de Assis Paes Sabadin pelo apoio e amizade. 
Aos Professores: Dr. Daniel Furtado Ferreira (UFLA); Dr. Júlio Sílvio de Souza Bueno Filho (UFLA) e Dr. Silvano César da Costa (UEL), as dúvidas sanadas.

Aos colegas do curso de pós-graduação em Estatística e Experimentação Agronômica.

A todos que de alguma forma auxiliaram para a realização deste trabalho. 


\section{SUMÁRIO}

Página

LISTA DE FIGURAS .................................................................... viii

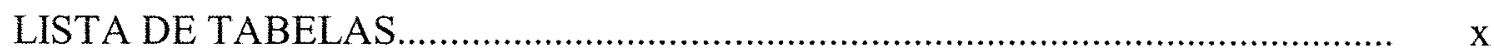

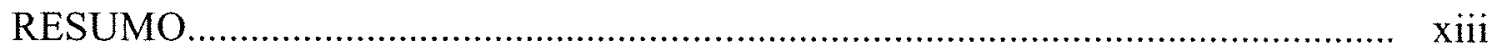

SUMMARY .................................................................................... Xvi

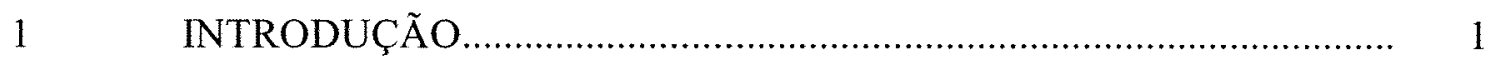

2 REVISÃO DE LITERATURA................................................... 4

$2.1 \quad$ Modelos lineares mistos.......................................................... 4

2.1.1 Estimação................................................................................ 5

2.1.2 Componentes de variância........................................................... 8

Transformações de dados............................................................. 12

2.2.1 Modelo linear geral e pressuposições................................................ 12

2.2.2 Escolha da transformação......................................................... 14

2.2.3 Transformação de Box-Cox ............................................................. 18

2.2.3.1 Estimação do parâmetro $\lambda$............................................................... 19

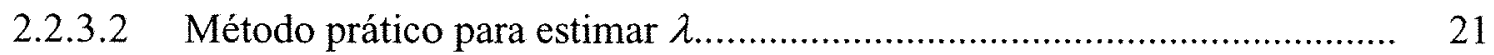

2.3 Modelos lineares generalizados..................................................... 22

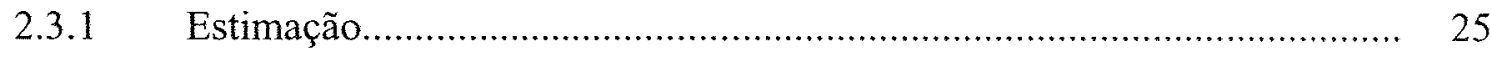

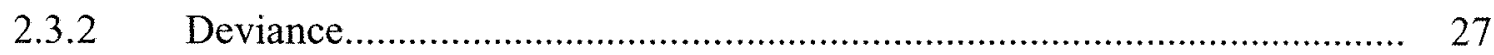

Função de quase-verossimilhança...................................................... 29

2.5 Modelos lineares generalizados mistos......................................... 31

2.5.1 Modelo....................................................................................... 31 


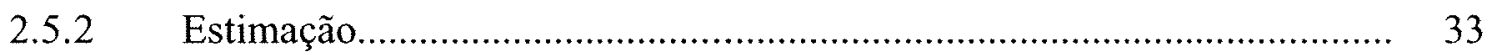

2.5.2.1 Método da máxima verossimilhança.......................................................... 34

2.5.2.1.1 Equações de verossimilhança........................................................................ 36

2.5.2.2 Método da quase-verossimilhança.......................................................... 39

2.6 Componentes de variância para dados binários.......................................... 44

2.7 Estimação do coeficiente de herdabilidade................................................ 49

2.7.1 Componentes da herdabilidade ................................................................ 50

2.7.2 Fatores que afetam a estimativa da herdabilidade................................... 51

2.7.3 Métodos de estimação do coeficiente de herdabilidade............................. 53

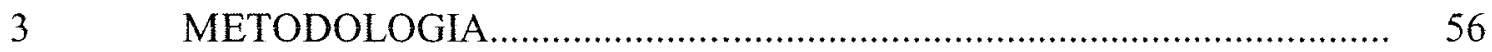

3.1 Simulação de dados.............................................................................. 56

3.2 Modelo linear misto clássico................................................................ 57

3.3 Modelo linear generalizado misto...................................................... 57

3.4 Coeficiente de herdabilidade ................................................................ 58

$3.5 \quad$ Comparação dos métodos ....................................................................... 59

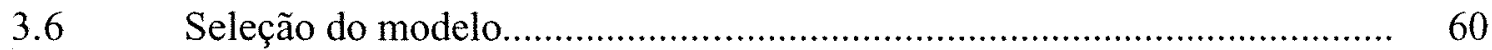

3.7 Dados de progênies de Eucalyptus grandis............................................ 60

$4 \quad$ RESULTADOS E DISCUSSÃO.......................................................... 63

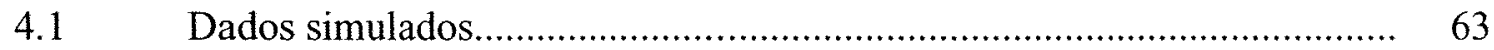

4.1.1 Modelo linear misto clássico................................................................ 63

4.1.2 Modelo linear generalizado misto............................................................ 68

4.1.3 Coeficiente de herdabilidade................................................................. 73

4.1.4 Comparação dos métodos........................................................................ 77

4.1.5 Seleção do modelo ................................................................................. 79

4.2 Dados de progênies de Eucalyptus grandis............................................. 84

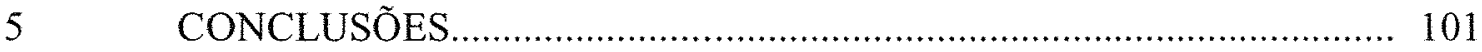

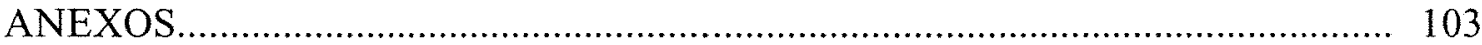

REFERÊNCIAS BIBLIOGRÁFICAS.............................................................. 116 


\section{LISTA DE FIGURAS}

Página

1 Coeficientes de herdabilidade para os experimentos simulados. MLMC Modelo linear misto clássico. MLGM - Modelo linear generalizado misto

2 Coeficientes de herdabilidade estimados para os experimentos simulados e valores de referência dos coeficientes de herdabilidade

3 Estimativas de efeitos fixos (blocos) para dados de plantas sobreviventes de Eucalyptus grandis, aos 5 anos de idade, num experimento em blocos ao acaso. MLMC - Modelo linear misto clássico. MLGM - Modelo linear generalizado misto

4 Efeitos aleatórios preditos para dados de plantas sobreviventes de Eucalyptus grandis, aos 5 anos de idade, num experimento em blocos ao acaso. MLMC Modelo linear misto clássico. MLGM - Modelo linear generalizado misto.

5 Proporções ajustadas de plantas sobreviventes de Eucalyptus grandis, aos 5 anos de idade, num experimento em blocos ao acaso. MLMC - Modelo linear misto clássico. MLGM - Modelo linear generalizado misto 
6 Gráficos para os resíduos, considerando os diferentes modelos de ajuste, de plantas sobreviventes de Eucalyptus grandis, aos 5 anos de idade, num experimento em blocos ao acaso

7 Gráficos $Q-Q$ Plot, considerando os diferentes modelos de ajuste, de plantas sobreviventes de Eucalyptus grandis, aos 5 anos de idade, num experimento em blocos ao acaso 


\section{LISTA DE TABELAS}

Página

1 Esquema da análise de variância das freqüências de um alelo de um dado loco

2 Esquema da análise de variância de um experimento envolvendo linhagens avaliadas em uma localidade, com repetições.

3 Esquema da análise de variância de um experimento envolvendo linhagens, localidades e repetições

4 Estimativas dos componentes de variância, obtidas pelo método da máxima verossimilhança, para os experimentos simulados

5 Estimativas dos componentes de variância e parâmetro de dispersão, obtidas pelo método da quase-verossimilhança (QV) e máxima verossimilhança (MV), para os experimentos simulados

6 Estimativas dos coeficientes de herdabilidade no sentido amplo para os experimentos simulados

7 Comparação das estimativas dos coeficientes de herdabilidade no sentido amplo, para os experimentos simulados, considerando os diferentes modelos 
8 Estimativas médias do coeficiente de herdabilidade, viés, erro padrão, mínimo, máximo, amplitude, para os experimentos simulados, e valores de referência dos coeficientes de herdabilidade

9 Estimativas dos componentes de variância e seus erros padrão, com respectivas probabilidades, obtidas pelos métodos de estimação: quaseverossimilhança $(\mathrm{QV})$ e máxima verossimilhança (MV), num modelo linear generalizado misto com função de ligação logística

10 Estimativas dos componentes de variância e seus erros padrão, com respectivas probabilidades, obtidas pelo método de estimação da máxima verossimilhança, num modelo linear misto clássico em diferentes escalas

11 Estimativas dos efeitos fixos (blocos), ajustados sob o enfoque de modelos lineares mistos clássicos, considerando as diferentes escalas, e admitindo-se um modelo linear generalizado misto com função de ligação logística, pelos métodos de estimação: quase-verossimilhança (QV) e máxima verossimilhança (MV)

12 Valores da correlação de Spearman para as estimativas de efeitos fixos (blocos), considerando os diferentes modelos de ajuste. MLMC - Modelo linear misto clássico. MLGM - Modelo linear generalizado misto.

13 Efeitos aleatórios preditos, ajustados sob o enfoque de modelos lineares mistos clássicos, considerando as diferentes escalas, e admitindo-se um modelo linear generalizado misto com função de ligação logística, pelos métodos de estimação: quase-verossimilhança (QV) e máxima verossimilhança (MV) 
14 Valores da correlação de Spearman para os efeitos aleatórios preditos, considerando os diferentes modelos de ajuste. MLMC - Modelo linear misto clássico. MLGM - Modelo linear generalizado misto

15 Valores da correlação de Spearman para as proporções ajustadas, considerando os diferentes modelos de ajuste. MLMC - Modelo linear misto clássico. MLGM - Modelo linear generalizado misto 


\title{
COMPARAÇÃO DE MODELOS MISTOS PARA DADOS DE PROPORÇÕES VISANDO À ESTIMAÇÃO DO COEFICIENTE DE HERDABILIDADE
}

\author{
Autor: TELDE NATEL CUSTÓDIO \\ Orientador: Prof. Dr. DÉCIO BARBIN
}

\section{RESUMO}

O objetivo deste trabalho foi apresentar um procedimento para comparação das estimativas do coeficiente de herdabilidade no sentido amplo, para dados provenientes de proporções, através das estimativas de componentes de variância obtidas pelo método da máxima verossimilhança. Considerou-se um modelo linear misto clássico, usando a escala original (proporções) e transformados. As transformações usadas foram arco seno $\sqrt{y / n}$ e BOX-COX. Considerou-se também um modelo linear generalizado misto com função de ligação logística, através dos métodos de estimação da quase-verossimilhança e máxima verossimilhança. Assim, adotou-se um modelo em blocos ao acaso, considerando-se blocos como sendo de efeito fixo e tratamentos como aleatório. Foram simulados 100 experimentos, com 20 tratamentos e 10 repetições (blocos). Foram feitas também, comparações das estimativas dos parâmetros de efeitos fixos, e efeitos aleatórios preditos, sob o enfoque de modelos lineares mistos clássicos, com os dados originais e transformados, utilizando-se as transformações arco seno $\sqrt{y / n}$ 
e BOX-COX, e também considerando-se um modelo linear generalizado misto com função de ligação logística, com dados provenientes de um experimento em blocos ao acaso, para avaliação de progênies de Eucalyptus grandis, aos 5 anos de idade, em que a variável resposta são proporções de plantas sobreviventes. Com base nos experimentos simulados, as conclusões obtidas foram: quando se têm dados de proporções, as estimativas dos coeficientes de herdabilidade no sentido amplo, não diferem na presença de transformações; as estimativas dos coeficientes de herdabilidade para os dados originais e transformados, obtidas através da teoria de modelos lineares mistos clássicos, diferiram das estimativas obtidas considerando-se um modelo linear generalizado misto; os métodos de estimação da quase-verossimilhança e máxima verossimilhança para o modelo linear generalizado misto, não diferiram quanto às estimativas dos coeficientes de herdabilidade; quando se têm dados de proporções, recomenda-se o uso de modelos lineares generalizados mistos, e dos métodos de estimação apresentados, para a estimação do coeficiente de herdabilidade. Em relação aos dados de proporções de plantas sobreviventes de Eucalyptus grandis, foi possível concluir que: o ajuste do modelo, através da teoria de modelos lineares mistos clássicos, com os dados transformados, por meio da transformação BOX-COX, não alterou a qualidade do ajuste em relação ao modelo ajustado com os dados na escala original, produzindo as mesmas estimativas para os efeitos fixos e predição para os efeitos aleatórios; os modelos ajustados sob o enfoque de modelos lineares mistos clássicos, com os dados na escala original, e transformados através da transformação BOX-COX, não se diferenciaram em relação aos modelos ajustados, considerando o enfoque de modelos lineares generalizados mistos, para ambos os métodos de estimação: quase-verossimilhança e máxima verossimilhança, produzindo estimativas para os efeitos fixos e predição para os efeitos aleatórios bastante semelhantes; o método de estimação da quaseverossimilhança, para o modelo linear generalizado misto, apresentou as mesmas estimativas dos efeitos fixos e predição para os efeitos aleatórios, em relação aos obtidos pelo método da máxima verossimilhança; o modelo ajustado por meio da transformação 
$\operatorname{arco} \operatorname{seno} \sqrt{y / n}$, considerando a abordagem de modelos lineares mistos clássicos, se diferenciou dos demais modelos, apresentando uma maior capacidade de predição. 


\title{
COMPARASION OF MIXED MODELS FOR PROPORTIONS DATA AIMING AT THE ESTIMATE OF HERITABILITY
}

\author{
Author: TELDE NATEL CUSTÓDIO \\ Adviser: Prof. Dr. DÉCIO BARBIN
}

\section{SUMMARY}

The objective of this work was to present a procedure for comparison of the estimates of heritability in the wide sense for data from proportions through estimates of variance components obtained by the method of maximum likelihood. A classic linear mixed model was considered by using the original (proportions) and transformed scale. The transformations used were $\arcsin \sqrt{y} / n$ and BOX-COX and a generalized linear mixed model with a function of logistic linkage through the estimation methods of quasi-likelihood and maximum likelihood. Thus, a randomized block model, considering blocks as being of fixed effect and treatments as random. 100 experiments were simulated, with 20 treatments and 10 replicates (blocks). Comparisons of the estimates of the parameters of fixed and predicted random effects under the approach of mixed classic linear models with the original data were also done and transformed, by utilizing the arc sine $\sqrt{y / n}$ transformation and BOX-COX, and also considering a generalized linear mixed model with the function of logistic link with 
data coming from a randomized block experiment for evaluation of Eucalyptus grandis progenies at five years old, in which the variant response are ratios of surviving plants. On the basis of the simulated experiments, the conclusions obtained were: when one has proportion data, the estimates of heritability do not differ in the presence of transformations; the estimates of the heritability for the original and transformed data obtained through the classic linear mixed model theory differ from the estimates obtained by considering a generalized linear mixed model; the methods of estimate of quasi-likelihood and maximum likelihood for the generalized linear mixed model did not differ as to the estimates of heritability; when one has proportion data, the use of generalized linear mixed models is recommended, and of the presented estimate methods, for the estimate of the heritability. Relative to the data of ratios of surviving Eucalyptus grandis plants, it was possible to conclude that: the fit of the model, through the theory of classic mixed linear models, with the data transformed through the BOXCOX transformation, has not altered the quality of the fit in relation to the model fitted with the data at the original scale, yielding the same estimates for the fixed effects and prediction for the random effects; the models fitted under the approach of classic mixed classic models, with the data at the original scale, and transformed through BOX-COX transformation, has not differed relative to the fitted models, considering the approach of generalized linear mixed model, for both the estimation methods: quasi-likelihood and maximum likelihood, yielding estimates for the fixed effects and prediction for the randomized effects quite similar; the method of quasi- likelihood estimation, for the generalized linear mixed model, presented the same estimates of the fixed effects and prediction for the randomized effects in relation to those obtained by the maximum likelihood method; the model fitted by means of the arc sine transformation $\sqrt{y / n}$, considering the approach of classic mixed linear models, distinguished from the other models, showing an increased prediction capacity. 


\section{INTRODUÇÃO}

Em muitas áreas da pesquisa científica, podem-se encontrar situações especiais de estudo, como é o caso das variáveis categorizadas ou binomiais, tais como a presença ou ausência de determinados atributos nos indivíduos. Em experimentos agronômicos podem se ter variáveis de interesse do tipo: porcentagem de germinação de sementes; proporção de plantas doentes; proporção de plantas sobreviventes; etc. Quando não se realiza um estudo adequado das características da distribuição de freqüências, esses tipos de dados são analisados, mediante a técnica da análise de variância, sem se ter a certeza de que todas as exigências necessárias para a aplicação dessa técnica foram satisfeitas. Como os modelos binomiais se apresentam nos casos em que a variável resposta representa uma proporção ou uma porcentagem de alguma característica observada da população em estudo, o interesse do pesquisador estará na estimação ou comparação das proporções. Mas, para que esses dados sejam avaliados mediante a técnica da análise de variância é necessário que eles atendam às pressuposições nas quais a análise se baseia. Nesse caso, tais variáveis não são bem explicadas pela teoria dos modelos lineares clássicos. Neles, um importante aspecto para a análise é a escala de medida.

Em certos casos quando não é possível satisfazer as pressuposições para análise de variância na própria escala observada, transformações têm sido feitas para uma escala mais apropriada com o objetivo de se conseguir homogeneidade de variâncias e uma distribuição aproximadamente normal.

Surge então, a procura de uma transformação de modo a proporcionar todas as condições favoráveis, para que a análise de variância realizada, forneça uma estimativa válida para a variância residual. 
É muito comum, na análise de variância para dados expressos em porcentagens, fazer-se a transformação dos dados arco seno $\sqrt{y / n}$ (sendo $y / n$ proporções), antes de se proceder à análise. Portanto, essa transformação é feita admitindo-se que a variável resposta segue uma distribuição binomial. Nesse caso, espera-se que a transformação homogeneizará a variância, que é uma das exigências para a validade dos testes estatísticos. Porém, em muitas situações práticas, a transformação necessária para estabilizar a variância pode não ser a mesma para obter uma resposta linear. Uma maneira de contornar o problema é o uso da teoria de modelos lineares generalizados.

Os modelos lineares generalizados foram desenvolvidos por Nelder \& Wedderburn (1972), visando à análise de dados associados a distribuições pertencentes à família exponencial com um parâmetro. Espera-se com isso, uma maior flexibilidade de análise. Tal idéia dispensa a suposição de que a variável resposta tenha uma distribuição normal e permite que essa siga qualquer distribuição pertencente à família exponencial na forma canônica.

Segundo McCullagh \& Nelder (1989), um modelo linear generalizado é composto por um componente aleatório associado à distribuição da variável resposta; um componente sistemático linear nos parâmetros, denominado preditor linear; uma função de ligação, que liga o componente aleatório ao componente sistemático, ou seja, relaciona a média ao preditor linear.

Incorporando efeitos aleatórios no preditor linear, têm-se os modelos lineares generalizados mistos. Nesse caso, o componente sistemático dos modelos lineares generalizados é inerente aos próprios modelos lineares mistos clássicos. Nesse sentido, a função de ligação deve ser incorporada aos estimadores dos efeitos fixos, preditores dos efeitos aleatórios e estimadores dos componentes de variância dos efeitos aleatórios, conforme Schall (1991).

Em Estatística Genética, os parâmetros genéticos obtidos com os dados na escala discreta podem ser utilizados visando à predição de valores genéticos e ganhos genéticos na própria escala observada. No entanto, nessa escala existe o inconveniente 
de se ter a dependência entre a média e a variância, de forma que a estimativa do coeficiente de herdabilidade, na escala discreta, é amplamente dependente da freqüência ou incidência média do fenótipo na população (Falconer, 1989).

Esse trabalho tem como objetivos:

i) simular experimentos em que a variável resposta são dados de proporções;

ii) estimar os componentes de variância envolvidos nos experimentos simulados, pelo método da máxima verossimilhança, sob o enfoque de modelos lineares mistos clássicos, com os dados originais (proporções) e transformados, utilizando-se as transformações arco seno $\sqrt{y / n}$ e BOX-COX e também considerando-se um modelo linear generalizado misto com função de ligação logística, através dos métodos de estimação da quaseverossimilhança e máxima verossimilhança;

iii) estimar e comparar os coeficientes de herdabilidade no sentido amplo para os experimentos simulados, através das estimativas dos componentes de variância obtidas, considerando-se: a teoria de modelos lineares mistos clássicos, com os dados originais (proporções) e transformados, utilizando-se as transformações arco seno $\sqrt{y / n}$ e BOX-COX; e pela teoria de modelos lineares generalizados mistos com função de ligação logística;

iv) fazer uma comparação das estimativas dos parâmetros de efeitos fixos, e efeitos aleatórios preditos, sob o enfoque de modelos lineares mistos clássicos, com os dados originais (proporções) e transformados, utilizando-se as transformações arco seno $\sqrt{y / n}$ e BOX-COX, e também considerando-se um modelo linear generalizado misto com função de ligação logística, com dados provenientes de um experimento em blocos ao acaso, para avaliação de progênies de Eucalyptus grandis, aos 5 anos de idade, em que a variável resposta são proporções de plantas sobreviventes. 


\section{REVISÃO DE LITERATURA}

\subsection{Modelos lineares mistos}

Num modelo linear, procura-se explicar a variável dependente mediante os efeitos diferenciais que se atribuem a uma outra série de variáveis independentes. Tais efeitos podem ser de natureza fixa ou aleatória. Segundo Searle (1987), modelos lineares nos parâmetros possuem pelo menos um efeito aleatório chamado erro experimental. Porém, se um modelo apresenta todos os demais componentes fixos, é chamado de modelo fixo, e se todos os demais fatores forem aleatórios, o modelo é chamado de aleatório. Quando um modelo apresenta ambos efeitos fixos e aleatórios, é denominado de modelo misto.

Para o modelo linear misto, a análise de variância apresenta algumas peculiaridades, como, por exemplo, a composição das esperanças matemáticas dos quadrados médios, cujo conhecimento permite o estabelecimento correto dos testes de hipóteses (Hicks, 1973). Caso o interesse do pesquisador esteja na estimação dos componentes de variância, métodos adequados devem ser utilizados (Searle et al., 1992).

Outro motivo para se adotar um modelo linear misto é a necessidade de se fazer a predição de efeitos aleatórios, na presença de efeitos fixos.

Um modelo linear misto clássico descrito em Searle et al. (1992) é dado por:

$$
y=X \beta+Z u+\varepsilon,
$$

em que: $y$ é o vetor $(n \times 1)$ das observações; $X$ é a matriz conhecida $(n \times p)$ de incidência dos efeitos fixos; $\beta$ é o vetor ( $p \times 1$ ) de efeitos fixos, desconhecido; $Z$ é a matriz conhecida $(n \times q)$ de incidência dos efeitos aleatórios; $u$ é o vetor $(q \times 1)$ de efeitos 
aleatórios, desconhecido; $\varepsilon$ é o vetor $(n \times 1)$ de erros aleatórios; $n$ é o número de observações, $p$ é o número de parâmetros de efeitos fixos; $q$ é o número de parâmetros de efeitos aleatórios.

Segundo esses mesmos autores, no modelo linear misto, assume-se que os efeitos aleatórios e os erros têm distribuição normal, com média zero, e matrizes de variâncias e covariâncias, $G$ e $R$, respectivamente. Assume-se também que os efeitos aleatórios e os erros são não correlacionados.

Tem-se, então, que:

$$
\begin{gathered}
u \sim \mathrm{N}(0 ; G) ; \\
\varepsilon \sim \mathrm{N}(0 ; R) ; \\
\operatorname{Cov}(u ; \varepsilon)=0 ; \\
\operatorname{Cov}\left(u_{i} ; u_{j}\right)=0, \text { para } i \neq j .
\end{gathered}
$$

Assim,

$$
\mathrm{E}(y)=X \beta \text { e } \operatorname{Var}(y)=Z G Z^{\prime}+R=V
$$

Logo,

$$
y \sim \mathrm{N}\left(X \beta ; Z G Z^{\prime}+R\right)
$$

\subsubsection{Estimação}

As pressuposições acerca da distribuição de $y, u$ e $\varepsilon$ podem ser descritas como:

$$
\left[\begin{array}{l}
y \\
u \\
\varepsilon
\end{array}\right] \sim N\left\{\left[\begin{array}{c}
X \beta \\
0 \\
0
\end{array}\right] ;\left[\begin{array}{ccc}
V & Z G & R \\
G Z^{\prime} & G & 0 \\
R & 0 & R
\end{array}\right]\right\} .
$$

As equações normais para o modelo misto podem ser obtidas: pela minimização do quadrado médio do erro (método dos mínimos quadrados generalizados); ou pela maximização da função densidade de probabilidade conjunta de $y$ e $u$ (método da máxima verossimilhança). 
Considerando o método da máxima verossimilhança, e admitindo-se que $u$ e $\varepsilon$ são normalmente distribuídos, tem-se que a distribuição condicional de $y \mid u$ é dada por:

$$
y \mid u \sim \mathrm{N}(X \beta+Z u ; R), \text { sendo } R=V-Z G Z \text {. }
$$

A função densidade de probabilidade de $y$ é dada por:

$$
f(y)=\frac{1}{(2 \pi)^{\frac{n}{2}}|V|^{\frac{1}{2}}} \exp \left\{-\frac{1}{2}\left[(y-X \beta)^{\prime} V^{-1}(y-X \beta)\right]\right\} .
$$

E a função densidade de probabilidade de $u$ é dada por:

$$
f(u)=\frac{1}{(2 \pi)^{\frac{n}{2}}|G|^{\frac{1}{2}}} \exp \left\{-\frac{1}{2}\left(u^{\prime} G^{-1} u\right)\right\} .
$$

A função densidade de probabilidade conjunta, $f(y, u)$, de acordo com Searle et al. (1992), pode ser escrita como o produto entre a função densidade de probabilidade condicional de $y$ dado $u$ e a função densidade de probabilidade de $u$.

Logo,

$$
f(y, u)=f(y \mid u) f(u)
$$

Assim,

$$
f(y, u)=\frac{1}{(2 \pi)^{\frac{n}{2}}|R|^{\frac{1}{2}}} \exp \left\{-\frac{1}{2}\left[(y-X \beta-Z u)^{\prime} R^{-1}(y-X \beta-Z u)\right]\right\} \frac{1}{(2 \pi)^{\frac{n}{2}} \mid G^{\mid \frac{1}{2}}} \exp \left\{-\frac{1}{2}\left(u^{\prime} G^{-1} u\right)\right\} \text {. }
$$

Portanto, tem-se que o logaritmo da função de verossimilhança $(\ell=\log [f(y, u)])$ é dado por:

$$
\begin{aligned}
\ell= & -\frac{1}{2} 2 n \log (2 \pi)-\frac{1}{2}(\log R|+\log G|)-\frac{1}{2}\left(y^{\prime} R^{-1} y-2 y^{\prime} R^{-1} X \beta-2 y^{\prime} R^{-1} Z u+2 \beta^{\prime} X^{\prime} R^{-1} Z u+\right. \\
& \left.+\beta^{\prime} X^{\prime} R^{-1} X \beta+u^{\prime} Z^{\prime} R^{-1} Z u+u^{\prime} G^{-1} u\right) .
\end{aligned}
$$

Derivando-se $\ell$ em relação a $\beta$ e a $u$, e igualando ambas as derivadas a zero, obtêm-se:

$$
\begin{gathered}
X^{\prime} R^{-I} X \hat{\beta}+X^{\prime} R^{-I} Z \hat{u}=X^{\prime} R^{-I} y \\
Z^{\prime} R^{-1} X \hat{\beta}+Z^{\prime} R^{-1} Z+G^{-1} \hat{u}=Z^{\prime} R^{-1} y
\end{gathered}
$$


Assim, tem-se que as equações normais para o modelo misto são dadas por:

$$
\left[\begin{array}{cc}
X^{\prime} R^{-1} X & X^{\prime} R^{-I} Z \\
Z^{\prime} R^{-I} X & Z^{\prime} R^{-I} Z+G^{-I}
\end{array}\right]\left[\begin{array}{l}
\hat{\beta} \\
\hat{u}
\end{array}\right]=\left[\begin{array}{c}
X^{\prime} R^{-I} y \\
Z^{\prime} R^{-1} y
\end{array}\right] .
$$

Esse sistema de equações permite obter soluções para os efeitos fixos $\beta$ e predições para os efeitos aleatórios $u$, cuja solução é:

$$
\begin{gathered}
\hat{\beta}=\left\{X^{\prime}\left[R^{-1}-R^{-1} Z\left(Z^{\prime} R^{-1} Z+G^{-1}\right)^{-1} Z^{\prime} R^{-1}\right] X\right\}^{-} X^{\prime}\left[R^{-1}-R^{-1} Z\left(Z^{\prime} R^{-1} Z+G^{-1}\right)^{-1} Z^{\prime} R^{-1}\right] y ; \\
\hat{u}=\left(Z^{\prime} R^{-1} Z+G^{-1}\right)^{-1} Z^{\prime} R^{-1}(y-X \hat{\beta}) .
\end{gathered}
$$

Algumas propriedades da solução para os efeitos fixos:

i) A solução $\hat{\beta}$, obtida pelas equações de modelo misto, é, também, uma solução de mínimos quadrados generalizados, ou seja,

$$
\hat{\beta}=\left(X^{\prime} V^{-1} X\right)^{-} X^{\prime} V^{-1} y,
$$

em que: $V^{-1}=R^{-1}-R^{-1} Z\left(Z^{\prime} R^{-1} Z+G^{-1}\right)^{-1} Z^{\prime} R^{-1}$.

ii) A variância de $\hat{\beta}$ é dada por:

$$
\operatorname{Var}(\hat{\beta})=\left[X^{\prime} R^{-1} X-X^{\prime} R^{-1} Z\left(Z^{\prime} R^{-1} Z+G^{-1}\right)^{-1} Z^{\prime} R^{-1} X\right]^{-} .
$$

Algumas propriedades da predição para os efeitos aleatórios:

i) O preditor $\hat{u}$ é o Melhor Preditor Linear Não-Viesado (BLUP) de $u$.

O termo predição refere-se a fatores aleatórios, e a Melhor Predição Linear Não-Viesada (BLUP) pode ser, definida como o resultado da regressão dos efeitos de um fator aleatório $u$, em função das observações $y$, corrigidas para os efeitos dos fatores fixos $X \beta$. 
ii) A variância de $\hat{u}$ é dada por:

$$
\begin{aligned}
\operatorname{Var}(\hat{u})= & G Z^{\prime}\left\{R^{-1}-R^{-1} Z\left(Z^{\prime} R^{-1} Z+G^{-1}\right)^{-1} Z^{\prime} R^{-1}-\left[R^{-1}-R^{-1} Z\left(Z^{\prime} R^{-1} Z+G^{-1}\right)^{-1} Z^{\prime} R^{-1}\right]\right. \\
& \left.X\left\{X^{\prime}\left[R^{-1}-R^{-1} Z\left(Z^{\prime} R^{-1} Z+G^{-1}\right)^{-1} Z^{\prime} R^{-1}\right] X\right\} X^{\prime}\left[R^{-1}-R^{-1} Z\left(Z^{\prime} R^{-1} Z+G^{-1}\right)^{-1} Z^{\prime} R^{-1}\right]\right\} G^{\prime} .
\end{aligned}
$$

iii) A variância do erro de predição é dada por:

$$
\begin{aligned}
\operatorname{Var}(u-\hat{u})= & G-G Z^{\prime}\left\{R^{-1}-R^{-1} Z\left(Z^{\prime} R^{-1} Z+G^{-1}\right)^{-1} Z^{\prime} R^{-1}-\left[R^{-1}-R^{-1} Z\left(Z^{\prime} R^{-1} Z+G^{-1}\right)^{-1} Z^{\prime} R^{-1}\right]\right. \\
& X\left\{X^{\prime}\left\{R^{-1}-R^{-1} Z\left(Z^{\prime} R^{-1} Z+G^{-1}\right)^{-1} Z^{\prime} R^{-1}\right\} X^{-1} X\left\{R^{-1}-R^{-1} Z\left(Z^{\prime} R^{-1} Z+G^{-1}\right)^{-1} Z^{\prime} R^{-1}\right]\right\} Z \mathrm{G}^{\prime} .
\end{aligned}
$$

Deve-se observar que o uso das equações de modelo misto, requer o conhecimento prévio das variâncias e covariâncias $(G$ e $R$ ) envolvidas no modelo (Searle et al., 1992). Esses parâmetros são, geralmente, desconhecidos, e o que se tem feito é substituí-los por suas estimativas.

\subsubsection{Componentes de variância}

O uso da metodologia de modelos mistos, para a predição de valores aleatórios, requer o conhecimento prévio dos componentes de variância e covariância envolvidos. Dessa forma, uma determinação precisa das estimativas desses componentes melhora tanto o processo de estimação, como o de predição, podendo resultar em alterações nas estimativas dos efeitos fixos, bem como nas predições dos efeitos aleatórios.

Os componentes de variância, que são variâncias associadas aos efeitos aleatórios de um modelo matemático, têm grande importância e aplicação tanto no melhoramento vegetal quanto animal, (obtenção de índices de repetibilidade, coeficiente de herdabilidade, etc), bem como em outras áreas, tais como médica e industrial.

Segundo Searle et al. (1992), os estimadores devem apresentar as seguintes propriedades: 
i) Não-viciado, tal que a esperança matemática do estimador seja o próprio parâmetro;

ii) Consistência, tal que, com o aumento do tamanho da amostra, a esperança do estimador converge para o parâmetro e a variância do estimador, para zero;

iii) Eficiência, tal que o estimador apresente variância mínima;

iv) Suficiência, tal que o estimador condense o máximo possível a informação contida na amostra e não seja função do parâmetro;

v) Completitude que está ligada à unicidade do estimador;

vi) Invariância a translação, tal que a estimação dos componentes de variância não seja afetada por mudanças nos efeitos fixos.

Diversas metodologias têm sido sugeridas para a estimação dos componentes de variância. Dentre elas, citam-se os métodos da máxima verossimilhança (ML) (Hartley \& Rao, 1967) e o da máxima verossimilhança restrita (REML) (Paterson $\&$ Thompson, 1971).

Searle et al. (1992) apresentam uma obra extensa sobre a estimação dos componentes de variância e análise de modelos mistos, tanto para dados balanceados como desbalanceados. Nessa obra, os autores apresentam, também, detalhes no que se refere à teoria dos métodos, além de apresentar ilustrações para cada método.

Barbin (1998) apresenta um trabalho sobre componentes de variância, enfatizando o método ANAVA.

O método da Máxima Verossimilhança (ML) para estimação de componentes de variância foi desenvolvido por Hartley \& Rao (1967), e consiste em maximizar a função densidade de probabilidade conjunta das observações, em relação aos efeitos fixos e aos componentes de variância dos efeitos aleatórios considerados no modelo, pressupondo normalidade.

No caso de dados desbalanceados, os estimadores ML apresentam as seguintes propriedades desejáveis: suficiência, consistência, eficiência e invariância a translação. 
Embora os estimadores de máxima verossimilhança apresentem propriedades estatísticas ótimas, eles apresentam vieses, por não considerar a perda no número de graus de liberdade, resultante da estimação dos efeitos fixos. A perda no número de graus de liberdade faz com que as estimativas dos componentes de variância obtidos por esse método, em geral, não coincidam com aqueles estimados pelo método do tipo ANAVA, mesmo quando os dados são balanceados.

Mesmo apresentando estimativas viciadas, o procedimento ML é computacionalmente simples e em certos casos, apresenta eficiência satisfatória. $\mathrm{O}$ vício pode ser considerável se o número de equações independentes (posto da matriz $X$ ) referente aos efeitos fixos for relativamente grande em relação ao número $N$ de observações.

$O$ viés presente nas estimativas obtidas pelo método ML pode ser removido, considerando-se apenas a parte da função de verossimilhança que independe dos efeitos fixos. Esse procedimento, pelo qual cada observação é dividida em duas partes independentes, uma referente aos efeitos fixos e outra, aos efeitos aleatórios, foi sugerido por Thompson (1962). Foram, porém, Patterson \& Thompson (1971) que desenvolveram o método de estimação para modelos mistos balanceados ou não, o qual foi denominado de Máxima Verossimilhança Restrita (REML). Assim, o método REML mantém as demais propriedades do método ML, é não viciado e permite também a imposição de restrições de não-negatividade. Para dados balanceados, os componentes de variância são os mesmos encontrados pelos métodos do tipo ANAVA. O procedimento REML é também denominado de máxima verossimilhança marginal e de máxima verossimilhança residual.

Os métodos REML e ML exigem normalidade para que os estimadores tenham propriedades desejáveis. Entretanto, tais estimadores podem ser robustos aos desvios da normalidade, gerando estimativas razoáveis mesmo quando a forma da distribuição não é especificada (Harville, 1977).

Nos casos em que os efeitos aleatórios são não correlacionados, os estimadores para os componentes de variância obtidos pelo método da máxima verossimilhança (ML) são dados conforme Fellner (1986; 1987) por: 


$$
\begin{gathered}
\hat{\sigma}_{u}^{2(\mathrm{~m}+1)}=\frac{\hat{u}^{\prime} \hat{u}}{q-\operatorname{tr} C_{22}^{-1} / \hat{\sigma}_{u}^{2(m)}} ; \\
\hat{\sigma}_{e}^{2(\mathrm{~m}+1)}=\frac{(y-X \hat{\beta}-Z \hat{u})(y-X \hat{\beta}-Z \hat{u})}{N-r(x)-q+\operatorname{tr} C_{22}^{-1} / \hat{\sigma}_{u}^{2(m)}} \hat{\sigma}_{e}^{2(m)} .
\end{gathered}
$$

Pelo método da máxima verossimilhança restrita (REML), os estimadores são dados por:

$$
\begin{gathered}
\hat{\sigma}_{u}^{2(\mathrm{~m}+1)}=\frac{\hat{u}^{\prime} \hat{u}}{q-\operatorname{tr} C^{22} / \hat{\sigma}_{u}^{2(m)}} ; \\
\hat{\sigma}_{e}^{2(\mathrm{~m}+1)}=\frac{(y-X \hat{\beta}-Z \hat{u})(y-X \hat{\beta}-Z \hat{u})}{N-r(x)-q+\operatorname{tr} C^{22} / \hat{\sigma}_{u}^{2(\mathrm{~m})}} \hat{\sigma}_{e}^{2(\mathrm{~m})},
\end{gathered}
$$

em que: $C_{22}$ é a partição da matriz dos coeficientes das equações do modelo misto, referente aos efeitos aleatórios; $C^{22}$ é a partição da inversa da matriz dos coeficientes das equações do modelo misto, referente aos efeitos aleatórios; $q$ é o número de níveis do efeito aleatório $u ; r(x)$ é o posto da matriz $X$; tr é o operador traço matricial; $\hat{\sigma}_{u}^{2(m)} \mathrm{e} \hat{\sigma}_{e}^{2(m)}$ são os valores correntes ou atuais (obtidos na iteração anterior) de $\hat{\sigma}_{u}^{2}$ e $\hat{\sigma}_{e}^{2}$, respectivamente.

Estes estimadores são idênticos aos apresentados por Dempster et al. (1977) e Harville (1977), referentes ao algoritmo EM.

Nos modelos lineares mistos, um importante aspecto para a análise é a escala de medida. Os problemas que podem surgir são devidos às seguintes razões: falta de normalidade dos erros, não-aditividade de unidades e efeitos de tratamentos e falta de homogeneidade de variâncias. Esses fenômenos são possivelmente relacionados.

Para tornar válida a aplicação desses modelos os pesquisadores têm adotado a mudança adequada da escala da variável mediante transformações nos dados para uma escala mais apropriada. 


\subsection{Transformações de dados}

As técnicas da análise de variância e de regressão são amplamente utilizadas em várias áreas da pesquisa científica. Entretanto, para aplicação dessas técnicas, faz-se necessário atender a certas pressuposições do modelo. Contudo, na prática, nem sempre tais pressuposições são satisfeitas.

Quando se trabalha com dados que não atendem às pressuposições do modelo para aplicação dessas técnicas, dois procedimentos devem ser seguidos: primeiro, devem-se buscar novos métodos de análise que dêem melhor ajuste; segundo, devem-se submeter os dados a uma transformação que atenda às pressuposições do modelo. Transformar os dados significa trocar a escala da variável original por uma outra escala.

A idéia básica é que, se para a variável original as pressuposições não são atendidas, pode existir uma transformação, tal que, na nova escala elas sejam razoavelmente atendidas.

As transformações, além de corrigir os desvios das pressuposições, podem também, ter outras aplicações, como linearizar um modelo de regressão ou eliminar a interação de um modelo de análise de variância, tornando-o aditivo, porém, em muitas situações práticas, a transformação necessária para estabilizar a variância pode não ser a mesma para obter uma resposta linear (Draper \& Smith, 1981). As transformações mais comumente usadas são discutidas em: Box \& Cox (1964); Steel \& Torrie (1980); Snedecor \& Cochran (1989).

\subsubsection{Modelo linear geral e pressuposições}

As técnicas de análise de variância e de regressão são tratadas através do modelo linear geral (Searle, 1987), dado por:

$$
y=X \beta+\varepsilon,
$$


em que: $y$ é o vetor $(n \times 1)$ das observações; $X$ é a matriz conhecida $(n \times p)$ de incidência dos parâmetros, obtida de acordo com o delineamento experimental e o modelo usado; $\beta$ é o vetor $(p \times 1)$ dos parâmetros, desconhecido; $\varepsilon$ é o vetor $(n \times 1)$ de erros aleatórios; $n$ o número de observações e $p$ o número de parâmetros do modelo.

Para o desenvolvimento teórico dessas técnicas são feitas certas pressuposições. Segundo Searle (1987) as pressuposições básicas que se deve admitir para a validade da análise de variância e de regressão, são as seguintes:

i) Aditividade: os efeitos dos fatores que ocorrem no modelo devem ser aditivos;

ii) Independência: os erros aleatórios devem ser independentes;

iii) Homocedasticidade ou homogeneidade de variâncias: os erros aleatórios devem possuir uma variância comum $\sigma^{2}$. Isso significa que a variabilidade das repetições de um tratamento deve ser semelhante a dos outros tratamentos, isto é, os tratamentos devem possuir variâncias homogêneas;

iv) Normalidade: os erros aleatórios devem possuir uma distribuição normal de probabilidade.

Essas pressuposições podem ser resumidas na forma: $\varepsilon \sim$ i.i.d. $\mathrm{N}\left(0 ; I \sigma^{2}\right)$, e como conseqüência $y \sim \mathrm{N}\left(X \beta ; I \sigma^{2}\right)$.

As suposições de homogeneidade de variâncias, normalidade e aditividade, nem sempre podem ser controladas pelo pesquisador. Já a suposição de independência dos erros, em geral, pode ser assegurada através de um esquema de aleatorização apropriado, na fase de planejamento do experimento.

Os testes apropriados para se verificar cada suposição do modelo linear são os seguintes, dentre outros:

i) Teste para não-aditividade:

- teste "F" da análise de variância;

- teste de Tukey para não-aditividade. 
ii) Teste para independência dos erros:

- teste de aleatoriedade;

- teste de correlação serial de Durbin-Watson.

iii) Teste de heterocedasticidade:

- teste de Cohran;

- teste de Hartley;

- teste de Bartlett.

iv) Teste de normalidade:

- teste de $\chi^{2}$ de Pearson;

- teste de Kolmogorov-Smirnov;

- teste de Lilliefors;

- teste de Shapiro-Wilk.

\subsubsection{Escolha da transformação}

O estudo das relações entre médias e variâncias de tratamentos pode sugerir uma possível transformação, de modo que se tenha variância constante e variâncias independentes das médias (Steel \& Torrie, 1980).

Assim, seja $Y$ uma variável aleatória com média, $\mathrm{E}(Y)=\mu$, e variância $\mathrm{V}(Y)$. Admite-se que a variância seja uma função da média, dada por:

$$
\mathrm{V}(Y)=f(\mu) .
$$

O que se pretende é determinar uma função de $Y, g(Y)$, que transforme $Y$ numa nova variável $Z$, com variância independente da média.

Tem-se, então que:

$$
Z=g(Y) .
$$

Para se determinar uma transformação adequada, usa-se expansão em série de Taylor de $1^{\underline{a}}$ ordem em $Z=\mathrm{g}(Y)$ em torno de $\mu$. 
Desse modo, tem-se que:

$$
Z \approx g(\mu)+g^{\prime}(\mu)(Y-\mu),
$$

cuja esperança é dada por:

$$
\mathrm{E}(Z) \approx g(\mu)
$$

em que $\mathrm{E}(Y-\mu)=0$.

Conseqüentemente,

$$
\begin{aligned}
\operatorname{Var}(Z) & =\mathrm{E}[Z-\mathrm{E}(Z)]^{2} \\
& =\mathrm{E}\left[g(\mu)+g^{\prime}(\mu)(Y-\mu)-g(\mu)\right]^{2} \\
& =\mathrm{E}\left[g^{\prime}(\mu)(Y-\mu)\right]^{2} \\
& =\left[g^{\prime}(\mu)\right]^{2} \mathrm{E}[Y-\mu]^{2} \\
& =\left[g^{\prime}(\mu)\right]^{2} \mathrm{~V}[Y] .
\end{aligned}
$$

Tem-se que:

$$
\mathrm{V}(Y)=f(\mu)
$$

Logo,

$$
\operatorname{Var}(Z)=\left[\mathrm{g}^{\prime}(\mu)\right]^{2} f(\mu)=C,
$$

em que $C$ é uma constante, independente de $\mu$.

Desse modo,

$$
\left[g^{\prime}(\mu)\right]^{2}=\frac{C}{f(\mu)}
$$

E assim,

$$
g^{\prime}(\mu)=\sqrt{\frac{C}{f(\mu)}} .
$$

Portanto,

$$
g(\mu)=\int \sqrt{\frac{C}{f(\mu)}} d \mu .
$$

Substituindo $\mu$ por $y$, obtém-se:

$$
g(y)=\sqrt{\operatorname{Var}(Z)} \int \frac{1}{\sqrt{f(y)}} d y .
$$


Para o caso em que a variável aleatória $Y$ segue uma distribuição binomial, tem-se que:

$$
\begin{gathered}
\mathrm{E}(Y)=\pi=\mu ; \\
\mathrm{V}(Y)=\frac{\pi(1-\pi)}{N}=\frac{\mu(1-\mu)}{N} .
\end{gathered}
$$

A variância de $Y$ é portanto uma função da média $\mu$.

Conforme (1), tem-se:

$$
\begin{aligned}
& g(y)=\sqrt{C} \int \frac{1}{\sqrt{\frac{y(1-y)}{N}}} d y \\
& =\sqrt{C} \int \frac{1}{\frac{\sqrt{y(1-y)}}{\sqrt{N}}} d y \\
& =\sqrt{C} \int \frac{\sqrt{N}}{\sqrt{y(1-y)}} d y \\
& =C \sqrt{N} \int \frac{1}{\sqrt{y(1-y)}} d y \\
& =C \sqrt{N} \int \frac{1}{\sqrt{y-y^{2}}} d y \text {. }
\end{aligned}
$$

Fazendo $y=t^{2}$, obtém-se:

$$
d y=2 t d t
$$

Assim,

$$
\begin{aligned}
g(y) & =C \sqrt{N} \int \frac{1}{\sqrt{t^{2}-t^{4}}} 2 t d t \\
& =C \sqrt{N} \int \frac{2 t}{\sqrt{t^{2}\left(1-t^{2}\right)}} d t \\
& =C \sqrt{N} 2 \int \frac{t}{t \sqrt{1-t^{2}}} d t \\
& =C \sqrt{N} 2 \int \frac{1}{\sqrt{1-t^{2}}} d t
\end{aligned}
$$




$$
=C \sqrt{N} 2 \text { arco seno } t+C_{1} .
$$

Tem-se que $t=\sqrt{y}$, e ignorando-se as constantes, a transformação adequada fica:

$$
g(y)=\operatorname{arcoseno} \sqrt{y}
$$

em que $y$ são proporções.

A variância de $Z$ é dada por:

$$
\begin{aligned}
\operatorname{Var}[Z] & =\left[g^{\prime}(y)\right]^{2} \operatorname{V}[Y] \\
& =\left[\frac{1}{\sqrt{1-y}} \frac{1}{2 \sqrt{y}}\right]^{2} \frac{\mu(1-\mu)}{N} \\
& =\frac{1}{(1-y)} \frac{1}{4 y} \frac{\mu(1-\mu)}{N} \\
& =\frac{1}{(1-\mu)} \frac{1}{4 \mu} \frac{\mu(1-\mu)}{N} .
\end{aligned}
$$

Logo,

$$
\operatorname{Var}[Z]=\frac{1}{4 N} .
$$

Portanto, observa-se pela expressão acima que a variância independe da média $\mu$.

Uma análise descritiva e exploratória dos dados, pode ajudar na escolha da transformação, mas, não existem regras gerais que garantam o sucesso na escolha da transformação.

As transformações mais utilizadas (Steel \& Torrie, 1980) são:

i) Transformação Logarítmica :

$$
\log (y+K) \text { ou } \ln (y+K), K \geq 0 .
$$

Recomendada quando é constatada uma certa proporcionalidade entre a média e a variância.

É muito usada em estudos biológicos, em estudos de efeito de drogas e também utilizada para se obterem relações lineares. 
ii) Transformação Raiz Quadrada:

$$
\sqrt{y+K}, K \geq 0 \text {. }
$$

Freqüentemente utilizada em dados provenientes de contagens, que geralmente seguem uma distribuição de Poisson, na qual a média é igual à variância.

iii) Transformação Recíproca:

$$
\frac{1}{y_{i}} \text { se } y_{i} \neq 0, i=1,2, \ldots, n \text {, ou } \frac{1}{\left(y_{i}+K\right)} \text { se } y_{i}=0 \text {. }
$$

Essa transformação é muito usada em análise de sobrevivência de plantas e animais, em estudos farmacológicos e em estudos de densidades de plantas por parcela.

iv) Transformação Angular:

$$
\operatorname{arcoseno} \sqrt{y / n} \text {, sendo } y / n \text { proporções. }
$$

Usada em dados expressos em porcentagens, que geralmente seguem uma distribuição binomial.

\subsubsection{Transformação de Box-Cox}

Box \& Cox (1964) propuseram uma família de transformações dada por:

$$
Y^{(\lambda)}=\left\{\begin{array}{l}
\frac{Y^{\lambda}-1}{\lambda}, \lambda \neq 0 \\
\log Y, \lambda=0
\end{array},\right.
$$

em que $Y$ é a variável resposta e $\lambda$ o parâmetro da transformação.

A idéia básica é considerar $\lambda$ como um parâmetro adicional e desconhecido do modelo, e estimá-lo pelos métodos padrões da inferência estatística. 
A suposição feita por Box \& $\operatorname{Cox}(1964)$ é que para algum $\lambda$, as observações transformadas por (2) podem ser tratadas como sendo normalmente distribuídas e independentes, com variância constante $\sigma^{2}$ e com esperança definida pelo modelo linear, dada por:

$$
\mathrm{E}\left[Y^{(\lambda)}\right]=X \beta
$$

Assim,

$$
Y^{(\lambda)} \sim \mathrm{N}\left(X \beta ; I \sigma^{2}\right)
$$

\subsubsection{Estimação do parâmetro $\lambda$}

Seja $Y=\left(y_{1}, y_{2}, \ldots, y_{n}\right)^{\prime}$ o vetor de observações da variável resposta. Suponha que para algum $\lambda$ desconhecido, o vetor de observações transformados $Y^{(\lambda)}=\left(y_{1}^{(\lambda)}, y_{2}^{(\lambda)}, \ldots, y_{n}^{(\lambda)}\right)^{\prime}$ satisfaça às pressuposições do modelo linear.

Assim, assumindo que, para uma escolha adequada de $\lambda$, o modelo linear é tal que:

$$
Y^{(\lambda)}=X \beta+\varepsilon,
$$

em que $\varepsilon \sim \mathrm{N}\left(0 ; I \sigma^{2}\right)$.

Considerando o método da máxima verossimilhança para estimação do parâmetro $\lambda$, a função de verossimilhança de $Y^{(\lambda)}$ é dada por:

$$
L\left(\beta, \sigma^{2}, \lambda ; Y\right)=\left(2 \pi \sigma^{2}\right)^{-\frac{n}{2}} \exp \left\{\frac{-\left(Y^{(\lambda)}-X \beta\right)^{\prime}\left(Y^{(\lambda)}-X \beta\right)}{2 \sigma^{2}}\right\} J(\lambda ; Y),
$$

em que $J(\lambda ; Y)$ é o jacobiano da transformação definido por:

$$
J(\lambda ; Y)=\prod_{i=1}^{n} \frac{d y_{i}^{(\lambda)}}{d y_{i}}=\prod_{i=1}^{n} y_{i}^{(\lambda-1)} .
$$

O logaritmo da função de verossimilhança fica:

$$
\ell\left(\beta, \sigma^{2}, \lambda ; Y\right)=-\frac{n}{2} \ln (2 \pi)-\frac{n}{2} \ln \left(\sigma^{2}\right)-\frac{1}{2 \sigma^{2}}\left[\left(Y^{(\lambda)}-X \beta\right)^{\prime}\left(Y^{(\lambda)}-X \beta\right)\right]+(\lambda-1) \sum_{i=1}^{\mathrm{n}} \ln \left(y_{i}\right) .
$$


Os estimadores de máxima verossimilhança dos parâmetros envolvidos em $\ell\left(\beta, \sigma^{2}, \lambda ; Y\right)$ são obtidos em duas etapas:

i) Para $\lambda$ fixo, estimam-se $\beta$ e $\sigma^{2}$.

Derivando-se (3) em relação a $\beta$ e a $\sigma^{2}$, obtém-se:

$$
\begin{gathered}
\frac{\partial \ell}{\partial \beta}=\frac{1}{\sigma^{2}} X^{\prime}\left[Y^{(\lambda)}-X \beta\right] ; \\
\frac{\partial \ell}{\partial \sigma^{2}}=-\frac{n}{2 \sigma^{2}}+\frac{1}{2\left(\sigma^{2}\right)^{2}}\left[Y^{(\lambda)}-X \beta\right]^{\prime}\left[Y^{(\lambda)}-X \beta\right] .
\end{gathered}
$$

Igualando-se a zero ambas as derivadas, tem-se que os estimadores são dados por:

$$
\begin{gathered}
\hat{\beta}(\lambda)=\left(X^{\prime} X\right)^{-1} X^{\prime} Y^{(\lambda)} \\
\hat{\sigma}^{2}(\lambda)=\frac{1}{n}\left[Y^{(\lambda)}-X \hat{\beta}\right] \cdot\left[Y^{(\lambda)}-X \hat{\beta}\right]=\frac{S(\lambda ; Y)}{n},
\end{gathered}
$$

em que $S(\lambda ; Y)$ é a soma de quadrados do resíduo referente a variável transformada.

Para $\lambda$ fixo, o máximo de $\ell\left(\beta, \sigma^{2}, \lambda ; Y\right)$ é dado por:

$$
\ell_{\max }(\lambda)=-\frac{n}{2} \ln (2 \pi)-\frac{n}{2} \ln \left(\hat{\sigma}^{2}(\lambda)\right)+(\lambda-1) \sum_{i=1}^{n} \ln \left(y_{i}\right)
$$

ii) A segunda etapa consiste em se determinar o estimador de máxima verossimilhança de $\lambda$.

Uma forma simples, porém equivalente, para $\ell_{\max }(\lambda)$ é obtida, trabalhando-se com a transformação normalizada, dada por:

$$
Z^{(\lambda)}=\frac{Y^{(\lambda)}}{J(\lambda ; Y)^{\frac{1}{n}}} .
$$

Como $J(\lambda ; Y)=\prod_{i=1}^{n} y_{i}^{(\lambda-1)}$, tem-se que $J(\lambda ; Y)^{\frac{1}{n}}=\dot{Y}^{\lambda-1}$, sendo $\dot{Y}$ a média geométrica das observações da variável $Y$.

Assim, 


$$
Z^{(\lambda)}=\frac{Y^{(\lambda)}}{\dot{Y}^{\lambda-1}}= \begin{cases}\frac{Y^{\lambda}-1}{\lambda \dot{Y}^{\lambda-1}} & , \lambda \neq 0 \\ \dot{Y} \log (Y) & , \lambda=0\end{cases}
$$

em que $\dot{Y}=\sqrt{\prod_{i=1}^{n} y_{i}}$

Na transformação normalizada, $J(\lambda ; Y)=1$ e, portanto, o logaritmo da função de verossimilhança parcialmente maximizada é dado por:

$$
\ell_{\max }(\lambda)=-\frac{n}{2} \log \left(\hat{\sigma}^{2}(Z)\right)=-\frac{n}{2} \log \left\{\frac{S(\lambda ; Z)}{n}\right\},
$$

em que $S(\lambda ; Z)$ é a soma de quadrados do resíduo referente a variável normalizada.

Assim, a estimativa de máxima verossimilhança, $\hat{\lambda}$, é o valor de $\lambda$ que maximiza $\ell_{\max }(\lambda)$, dado em (4) ou, equivalentemente, minimiza $S(\lambda ; Z)$.

Box \& Cox (1964) sugerem uma solução numérica mais simples possível. Para vários valores de $\lambda$ num intervalo conveniente, determina-se $\ell_{\max }(\lambda)$ em função de $\lambda$ e então determina-se graficamente o valor de $\lambda$ que minimiza essa função. Esse, então, será o estimador de máxima verossimilhança $\hat{\lambda}$, ficando assim determinada a transformação de BOX-COX.

\subsubsection{Método prático para estimar $\lambda$}

Na aplicação do procedimento de BOX-COX, Draper \& Smith (1981) sugerem que se tomem de 11 a 21 valores de $\lambda$, no intervalo de $[-2 ; 2]$. Nas proximidades do ponto de máximo, pode-se tomar valores adicionais de $\lambda$ para tornar a determinação de $\hat{\lambda}$ mais precisa. Entretanto, na prática, nem sempre se utiliza o valor de $\hat{\lambda}$ obtido, mas sim, um valor mais próximo da seqüência $\ldots,-2,-\frac{3}{2},-1,-\frac{1}{2}, 0, \frac{1}{2}$, $1, \frac{3}{2}, 2, \ldots$ 
Esse procedimento facilita a interpretação da variável transformada. Essa substituição é bastante razoável quando o valor adotado estiver contido no intervalo de confiança para $\lambda$.

A construção do intervalo de confiança para $\lambda$, baseia-se no fato de que $-2 \log \Lambda$, tem distribuição aproximadamente $\chi^{2}$, em que $\Lambda$ é a razão de verossimilhanças (Mood et al., 1974). Assim, um intervalo de confiança, com um coeficiente de confiança de $100(1-\alpha) \%$ para $\lambda$, é dado por:

$$
2\left[\ell_{\max }(\hat{\lambda})-\ell_{\max }(\lambda)\right] \leq \chi_{1, \alpha}^{2},
$$

em que $\chi_{1}^{2}$ é o quantil de ordem 1 - $\alpha$ da distribuição $\chi^{2}$ com um grau de liberdade.

Uma outra alternativa de transformação é através do uso da teoria de modelos lineares generalizados, que também envolve uma transformação, não dos dados, mas da média, e assim essa transformação é conhecida como função de ligação.

\subsection{Modelos lineares generalizados}

Os modelos lineares generalizados foram desenvolvidos para análise de dados associados a distribuições pertencentes à família exponencial com um parâmetro. Essa classe de modelos foi proposta por Nelder \& Wedderburn (1972). Tais modelos são extensões dos modelos lineares clássicos para casos em que as pressuposições do modelo são violadas. Os modelos lineares generalizados admitem qualquer distribuição de probabilidade pertencente à família exponencial na forma canônica de distribuições para os dados. McCullagh \& Nelder (1989) apresentam uma discussão completa da teoria e aplicação de modelos lineares generalizados.

Segundo McCullagh \& Nelder (1989), um modelo linear generalizado pode ser definido por três componentes:

i) Um componente aleatório representado pelas variáveis aleatórias independentes $Y_{l}, Y_{2}, \ldots, Y_{n}$ pertencentes a uma mesma distribuição que faz parte da família exponencial na forma canônica com médias $\mu_{l}, \mu_{2}, \ldots, \mu_{n}$, e 
um parâmetro de escala constante, $\phi>0$. A função densidade de probabilidade de $Y_{i}$ é dada por:

$$
f\left(y_{i} ; \theta_{i}, \phi\right)=\exp \left\{\frac{1}{a_{i}(\phi)}\left[y_{i} \theta_{i}-b\left(\theta_{i}\right)\right]+c\left(y_{i} ; \phi\right)\right\},
$$

em que: $\theta_{i}$ é o parâmetro canônico; $a_{i}(\phi), b\left(\theta_{i}\right)$ e $c\left(y_{i} ; \phi\right)$ são funções conhecidas.

Em geral, nos modelos pertencentes à família exponencial a função $a_{i}(\phi)$ pode ser escrita na forma:

$$
a_{i}(\phi)=\frac{\phi}{w_{i}},
$$

sendo $w_{i}$ o peso "a priori", geralmente igual a 1.

Pode-se mostrar que a média e a variância de uma variável aleatória $Y_{i}$ cuja distribuição pertença à família exponencial, na forma dada em (5), são dadas por:

$$
\mathrm{E}\left(Y_{i}\right)=\mu_{i}=\frac{d b\left(\theta_{i}\right)}{d \theta_{i}} \mathrm{e} \operatorname{Var}\left(Y_{i}\right)=\sigma_{i}^{2}=a_{i}(\phi) \frac{d^{2} b\left(\theta_{i}\right)}{d \theta_{i}^{2}} .
$$

Além disso, $\frac{d^{2} b\left(\theta_{i}\right)}{d \theta_{i}^{2}}=\frac{d \mu_{i}}{d \theta_{i}}$ é uma função de $\mu_{i}$ e é representada por $V\left(\mu_{i}\right)$, chamada função de variância.

Assim, a variância de $Y_{i}$ pode ser expressa por:

$$
\operatorname{Var}\left(Y_{i}\right)=\sigma_{i}^{2}=a_{i}(\phi) V\left(\mu_{i}\right) .
$$

Pertencem à família exponencial na forma canônica, as distribuições: normal, binomial, Poisson, binomial negativa, gama, normal inversa.

ii) Um componente sistemático chamado de preditor linear, na qual as variáveis explicativas entram na forma de uma soma linear de seus efeitos, dado por:

$$
\eta=X \beta \text {, }
$$

em que: $\eta=\left(\eta_{1}, \ldots, \eta_{n}\right)^{\prime}$ é o preditor linear $\left(\begin{array}{lll}n & x & l\end{array}\right) ; X=\left(x_{l}, \ldots, x_{n}\right)^{\prime}$ é a matriz $(n x p)$ conhecida de incidência dos parâmetros; $\beta=\left(\beta_{l}, \ldots, \beta_{p}\right)^{\prime}$ é o vetor $(p \times l)$ de parâmetros desconhecidos. 
iii) Uma função de ligação $g($. ) que liga o componente aleatório ao componente sistemático, ou seja, relaciona a média ao preditor linear.

Tem-se, então que:

$$
\eta_{i}=g\left(\mu_{i}\right)
$$

sendo $g\left(\right.$. ) uma função monótona e diferenciável e $\mu_{i}=\mathrm{E}\left(Y_{i}\right)$.

Além do mais, assume-se que a média transformada segue um modelo linear, ou seja, $\eta_{i}=x_{i}^{\prime} \beta$.

Sendo a função de ligação uma função monótona, então, pode-se invertêla, obtendo-se:

$$
\mu_{i}=g^{-1}\left(\eta_{i}\right)=g^{-1}\left(x_{i}^{\prime} \beta\right),
$$

em que $g^{-1}\left(\eta_{i}\right)$ é a função de ligação inversa.

Nota-se que não se transforma a variável resposta $Y_{i}$, mas seu valor esperado $\mu_{i}$.

Um modelo linear clássico pode ser descrito como um modelo linear generalizado com distribuição normal e função de ligação identidade, tal que $\eta_{i}=\mu_{i}$.

Portanto, $\eta_{i}$ é o mesmo que $\theta_{i}$, ou seja, o parâmetro canônico ou natural da distribuição normal. Assim, quando a forma da função de ligação no preditor linear $\eta_{i}$ é a mesma que o parâmetro canônico $\theta_{i}$, a função de ligação é chamada de canônica.

Uma característica de funções de ligação canônicas, é que existe uma estatística suficiente minimal para $\beta$, isto é, todas as informações sobre $\beta$ estão contidas numa função dos dados, de mesma dimensão que $\beta$.

Os modelos lineares generalizados com essa característica possuem propriedades importantes, tais como a concavidade do logaritmo da função de verossimilhança de um modelo linear generalizado, que garante a unicidade da estimativa de máxima verossimilhança de $\beta$. Outra conseqüência é que resultados assintóticos são obtidos mais facilmente.

As funções de ligação canônicas para as distribuições pertencentes à família exponencial, na forma dada em (5), são dadas (Demétrio, 2001) por: 
- Normal: Identidade: $\eta_{i}=\mu_{i}$;

- Poisson: Logarítmica: $\eta_{i}=\ln \left(\mu_{i}\right)$;

- Binomial: Logística: $\eta_{i}=\ln \left(\frac{\mu_{i}}{1-\mu_{i}}\right)$;

- Gama: Recíproca: $\eta_{i}=\frac{1}{\mu_{i}}$;

- Normal Inversa: Recíproca ${ }^{2}: \eta_{i}=\frac{1}{\mu_{i}^{2}}$.

Em resumo, na definição de um modelo linear generalizado, são fundamentais a definição da distribuição da variável resposta, a definição do preditor linear ou matriz do modelo e a definição da função de ligação.

Segundo Cordeiro (1986), a palavra generalizado significa uma distribuição mais ampla do que a normal para a variável resposta e uma função nãolinear conectando a média dessa variável com a parte determinística do modelo.

\subsubsection{Estimação}

Num modelo linear generalizado, Nelder \& Wedderburn (1972) afirmam que a idéia básica é estimar os parâmetros do modelo linear usando a função de verossimilhança baseada na distribuição dos dados. Na prática, a estimação dos parâmetros dos modelos lineares generalizados assemelha-se com mínimos quadrados generalizados.

O logaritmo da função de verossimilhança de um modelo linear generalizado com respostas independentes pode ser expresso na forma:

$$
\ell(\theta ; y)=\sum_{i=1}^{n}\left\{\frac{1}{a_{i}(\phi)}\left[y_{i} \theta_{i}-b\left(\theta_{i}\right)\right]+c\left(y_{i} ; \phi\right)\right\} .
$$

Nelder \& Wedderburn (1972) mostraram que as estimativas de máxima verossimilhança para os parâmetros do modelo, usando o método escore de Fisher, podem ser obtidas por: 


$$
\beta^{(\mathrm{m}+1)}=\left(X^{\prime} W^{(\mathrm{m})} X\right)^{-1} X^{\prime} W^{(\mathrm{m})} z^{(\mathrm{m})},
$$

em que:

- $\quad X=\left(x_{1}, \ldots, x_{n}\right)^{\prime}$ é a matriz ( $\left.n \times p\right)$ conhecida de incidência dos parâmetros;

- $W=\operatorname{diag}\left(W_{1}, \ldots, W_{n}\right)$, sendo $W_{i}=\frac{w_{i}}{V\left(\mu_{i}\right)}\left(\frac{d \mu_{i}}{d \eta_{i}}\right)^{2}, i=1, \ldots, n$ e $w_{i}$ o peso "a priori";

- $z=\eta+\Delta(y-\mu)$, sendo $\Delta=\operatorname{diag}\left(\frac{d \eta_{1}}{d \mu_{1}}, \frac{d \eta_{2}}{d \mu_{2}}, \ldots, \frac{d \eta_{n}}{d \mu_{n}}\right)=\operatorname{diag}\left(\frac{d \eta_{i}}{d \mu_{i}}\right)$, e $z$ a variável dependente ajustada.

Uma importante característica prática dos modelos lineares generalizados, é que estes podem ser ajustados usando o mesmo algoritmo. Tal algoritmo é uma forma de mínimos quadrados reponderados iterativamente.

Dada uma estimativa inicial dos parâmetros $\hat{\beta}$, estima-se o preditor linear, $\hat{\eta}_{i}=x_{i}^{\prime} \hat{\beta}$, e utiliza-se essa estimativa para se obterem os valores ajustados $\hat{\mu}_{i}=g^{-1}\left(\hat{\eta}_{i}\right)$. Com essas estimativas, calcula-se a variável dependente ajustada, $z_{i}$, dada por:

$$
z_{i}=\hat{\eta}_{i}+\left(y_{i}-\hat{\mu}_{i}\right) \frac{d \eta_{i}}{d \mu_{i}}
$$

O próximo passo é calcular os pesos,

$$
W_{i}=\frac{w_{i}}{\mathrm{~V}\left(\mu_{i}\right)}\left(\frac{d \mu_{i}}{d \eta_{i}}\right)^{2}
$$

Finalmente, obtém-se uma estimativa de $\beta$, usando a variável dependente ajustada $z_{i}$ nos preditores $x_{i}$ com pesos $W_{i}$, isto é, calculando as estimativas de mínimos quadrados ponderados, através de:

$$
\beta^{(\mathrm{m}+\mathrm{l})}=\left(X^{\prime} W^{(\mathrm{m})} X\right)^{-1} X^{\prime} W^{(\mathrm{m})} z^{(\mathrm{m})} .
$$

O procedimento é repetido até que a convergência seja alcançada. 


\subsubsection{Deviance}

Segundo McCullagh \& Nelder (1989), o processo para se ajustar um modelo aos dados pode ser visto como um meio de trocar valores de um conjunto de dados $y$ por um conjunto de valores ajustados $\hat{\mu}$ provenientes de um modelo envolvendo, geralmente, um pequeno número de parâmetros. Normalmente, os $\mu$ 's não serão iguais aos y's exatamente, e a questão é em quanto eles são discrepantes.

Considerando $n$ observações, podem-se ajustar modelos com até $n$ parâmetros. $\mathrm{O}$ modelo mais simples é chamado modelo nulo e tem um único parâmetro $\mu$ comum para todos os $y$ 's. Esse modelo concentra toda a variação dos $y$ 's no componente aleatório. Em outro extremo, tem-se o modelo completo com $n$ parâmetros, um para cada observação, e os $\mu$ 's obtidos por esse modelo representam os dados exatamente. Tem-se que o modelo completo concentra toda a variação dos y's no componente sistemático, nada sobrando para o componente aleatório e, portanto, ajustase perfeitamente (McCullagh \& Nelder, 1989).

O que se observa na prática é que o modelo nulo é simples demais e o modelo completo é não informativo, porque não representa os dados, apenas os repete. Contudo, o modelo completo fornece uma base para se medir a discrepância para um modelo intermediário contendo $p$ parâmetros.

Além de sua utilidade na estimação, o princípio da verossimilhança também permite comparar a adequabilidade de vários modelos, desde que tenham uma estrutura hierárquica ou aninhada.

Nelder \& Wedderburn (1972) propuseram uma medida de discrepância para a verificação da qualidade do ajuste de um modelo linear generalizado chamada "deviance". Seja $\ell(\hat{\mu}, \phi, y)$ o logaritmo da função de verossimilhança maximizada em relação a $\beta$ para um valor fixo do parâmetro de dispersão $\phi$. Tem-se que a função de verossimilhança para o modelo completo contendo $n$ parâmetros é dada por $\ell(y, \phi, y)$, a qual é comumente finita. A discrepância de um ajuste é proporcional a duas vezes a 
diferença entre o máximo do logaritmo da função de verossimilhança do modelo completo, $\ell(y ; y)$, e a do modelo sob pesquisa, $\ell(\hat{\mu} ; y)$, dada por:

$$
D(y ; \hat{\mu})=2[\ell(y ; y)-\ell(\hat{\mu} ; y)]=\frac{S(y ; \hat{\mu})}{\phi},
$$

em que:

- $D(y ; \hat{\mu})$ é chamada de "scaled deviance" e $S(y ; \hat{\mu})$, "deviance";

$-\quad \ell(y ; y)=\frac{1}{\phi} \sum_{i=1}^{n}\left\{w_{i}\left[y_{i} \tilde{\theta}_{i}-b\left(\widetilde{\theta}_{i}\right)\right]+c\left(y_{i} ; \phi\right)\right\}$

$-\ell(\hat{\mu} ; y)=\frac{1}{\phi} \sum_{i=1}^{n}\left\{w_{i}\left[y_{i} \hat{\theta}_{i}-b\left(\hat{\theta}_{i}\right)\right]+c\left(y_{i} ; \phi\right)\right\}$

- $\tilde{\theta}_{i}=\tilde{\theta}\left(y_{i}\right)$ e $\hat{\theta}_{i}=\hat{\theta}\left(\hat{\mu}_{i}\right)$, as estimativas do parâmetro canônico sob os modelos completo e pesquisa, respectivamente.

Assim,

$$
D(y ; \hat{\mu})=\frac{2}{\phi} \sum_{i=1}^{n} w_{i}\left[y_{i}\left(\widetilde{\theta}_{i}-\hat{\theta}_{i}\right)-b\left(\widetilde{\theta}_{i}\right)+b\left(\hat{\theta}_{i}\right)\right] .
$$

A "deviance" é uma generalização da soma de quadrados do resíduo na análise de variância, sendo igual a essa no caso da distribuição normal.

A "deviance" apresenta algumas propriedades simples que indicam a sua utilidade como uma medida da qualidade do ajustamento. Se um modelo se ajusta perfeitamente aos dados, isto é, $\hat{\mu}=y$, a "deviance" é zero; caso contrário, é positiva. Segundo Firth (1991), uma vez que a maximização da função de verossimilhança de qualquer modelo é equivalente à minimização da "deviance", o procedimento de máxima verossimilhança propicia o melhor ajuste pelo critério da "deviance".

Segundo McCullagh \& Nelder (1989), a "deviance" tem uma distribuição desconhecida, mas sob determinadas condições de regularidade para as distribuições binomial e Poisson, para grandes amostras, essa apresenta uma distribuição assintoticamente $\chi_{n-p}^{2}$ (Qui-Quadrado $\operatorname{com} n-p$ graus de liberdade), sendo $n$ igual ao número de observações e $p$ igual ao número de parâmetros envolvidos no modelo. Para a distribuição normal a "scaled deviance" tem distribuição $\chi^{2}$ exata. 
Para se testar o ajuste de um modelo linear generalizado, compara-se o valor calculado da "scaled deviance" com os percentis da distribuição $\chi_{n-p ; \alpha}^{2}$.

Nos casos em que é possível fazer uma aproximação pela distribuição de Qui-Quadrado, observa-se que, se $D(y ; \hat{\mu}) \leq \chi_{n-p ; \alpha}^{2}$, pode-se aceitar a hipótese de que existam evidências a um nível aproximado de $100 \%$ de probabilidade de que o modelo proposto se ajusta bem aos dados.

Outra medida de discrepância para a verificação da qualidade do ajuste de um modelo linear generalizado é a estatística $X^{2}$ generalizada de Pearson, dada por:

$$
X^{2}=\sum_{i=1}^{n} w_{i} \frac{\left(y_{i}-\hat{\mu}_{i}\right)^{2}}{V\left(\hat{\mu}_{i}\right)}
$$

em que $V\left(\hat{\mu}_{i}\right)$ é a função de variância estimada sob o modelo que está sendo ajustado.

Conforme McCullagh \& Nelder (1989), $\ell(\hat{\mu}, \phi ; y)$ equivale ao logaritmo da função de verossimilhança maximizada para um valor fixado do parâmetro de dispersão $\phi$. Para dados binários, $\phi$ é conhecido e igual a 1. Entretanto, na prática, os dados podem exibir algum grau de superdispersão $(\phi>1)$ ou subdispersão $(\phi<1)$. Segundo Lindsey (1997), o fato de $\phi$ ser diferente de 1 pode ser explicado pelo não atendimento das suposições assumidas para que determinado conjunto de dados tenha distribuição binomial. Nesse caso, quebra-se a dependência original existente entre a média $(\mu)$ e a variância $[\mu(1-\mu)]$ de uma distribuição Bernoulli. Na presença de sub ou superdispersão, uma correção visando à realização de inferências aproximadas refere-se à multiplicação da variância $[\mu(1-\mu)]$ por uma estimativa $\hat{\phi}$ de $\phi$ (Firth, 1991).

\subsection{Função de Quase-Verossimilhança}

Nos modelos lineares generalizados, a estimação dos parâmetros do modelo é feita usando a função de verossimilhança dos dados. Em muitas situações práticas, é difícil especificar a distribuição dos dados, tornando o uso do método da 
máxima verossimilhança impossivel de ser implementado. Em tais situações, o uso de uma função de quase-verossimilhança torna-se necessário.

A função de quase-verossimilhança foi introduzida por Wedderburn (1974) e é discutida em detalhes por McCullagh (1983) e McCullagh \& Nelder (1989).

Segundo Wedderburn (1974), a função de quase-verossimilhança pode ser interpretada como uma generalização dos modelos lineares generalizados em que se assume uma função de variância para a variável resposta, bem como uma relação funcional entre a média e o vetor de parâmetros $\beta$. No entanto, não se requer mais o conhecimento da distribuição da variável resposta.

Wedderburn (1974) define a função de quase-verossimilhança da seguinte maneira: Suponha que $y_{i}(i=1,2, \ldots, n)$ seja um conjunto de observações com média, $\mathrm{E}\left(y_{i}\right)=\mu_{i}$ e variância $\operatorname{Var}\left(y_{i}\right) \propto \mathrm{V}\left(\mu_{i}\right)$, em que $\mathrm{V}\left(\mu_{i}\right)$ é uma função conhecida da média $\mu_{i}$, chamada de função de variância. Suponha também que $\mu_{i}$ seja uma função de um conjunto de parâmetros $\beta_{1}, \beta_{2}, \ldots, \beta_{p}$, isto é, $\mu_{i}=g^{-1}\left(\eta_{i}\right)$, sendo $\eta_{i}=x_{i}^{\prime} \beta$ e $g^{-1}\left(\eta_{i}\right)$ a função de ligação inversa de um modelo linear generalizado. A função de quaseverossimilhança é definida pela relação:

$$
Q\left(\mu_{i} ; y_{i}\right)=\int_{y_{i}}^{\mu_{i}} \frac{y_{i}-t}{\phi V(t)} d t .
$$

Segundo McCullagh \& Nelder (1989), as estimativas de quaseverossimilhança para o vetor de parâmetros $\beta$ podem ser obtidas pelo método Escore de Fisher, resultando no seguinte processo iterativo:

$$
\beta^{(\mathrm{m}+1)}=\beta^{(\mathrm{m})}+\left[D^{(\mathrm{m})^{\prime}} V^{-(\mathrm{m})} D^{(\mathrm{m})}\right]^{-1} D^{(\mathrm{m})}[y-\mu]^{(\mathrm{m})},
$$

em que:

$$
\begin{aligned}
& -\quad \mathrm{m}=0,1,2, \ldots ; \\
& -\quad D=\frac{\partial \mu}{\partial \beta}=W^{1 / 2} V^{1 / 2} X ; \\
& -\quad \mu=\left(\mu_{1}, \mu_{2}, \ldots, \mu_{n}\right)^{\prime} ; \\
& -\quad \eta=\left(\eta_{1}, \eta_{2}, \ldots, \eta_{n}\right)^{\prime} ;
\end{aligned}
$$


- $y=\left(y_{1}, y_{2}, \ldots, y_{n}\right)^{\prime}$;

- $V=\operatorname{diag}\left(V_{\mathrm{l}}, V_{2}, \ldots, V_{n}\right)$;

- $W=\operatorname{diag}\left(w_{1}, w_{2}, \ldots, w_{n}\right)$, sendo $w_{i}=\frac{\left(\frac{d \mu_{i}}{d \eta_{i}}\right)^{2}}{V_{i}} ;$

- $X=\left(x_{1}, x_{2}, \ldots, x_{\mathrm{n}}\right)^{\prime}$.

A principal conseqüência prática da função de quase-verossimilhança é que se pode usar a teoria e métodos de modelos lineares generalizados para qualquer variável aleatória, em que se pode determinar $Q\left(\mu_{\mathrm{i}} ; y_{i}\right)$, ou seja, cuja relação entre média e variância seja conhecida.

\subsection{Modelos lineares generalizados mistos}

Os modelos lineares generalizados mistos são uma extensão dos modelos lineares generalizados e modelos lineares mistos clássicos. O componente sistemático dos modelos lineares generalizados é inerente aos próprios modelos lineares mistos clássicos. Nesse sentido, a função de ligação deve ser incorporada aos estimadores dos efeitos fixos, preditores dos efeitos aleatórios e estimadores dos componentes de variância dos efeitos aleatórios, conforme Schall (1991).

\subsubsection{Modelo}

Considere a distribuição condicional de $y$ dado $u$, em que $y$ é o vetor da variável resposta, composto por elementos condicionalmente independentes (não necessariamente) com distribuição pertencente à família exponencial.

Ássim,

$$
y_{i} \mid u \sim \text { indep. } f_{Y_{i} \mid U}\left(y_{i} \mid u\right),
$$




$$
f_{Y_{,}, U}\left(y_{i} \mid u\right)=\exp \left\{\frac{1}{a_{i}(\phi)}\left[y_{i} \theta_{i}-b\left(\theta_{i}\right)\right]+c\left(y_{i} ; \phi\right)\right\} .
$$

Além disso, tem-se que:

$$
\begin{gathered}
\mathrm{E}\left[y_{i} \mid u\right]=\mu_{i}=g^{-1}\left(\mu_{i}\right) ; \\
\operatorname{Var}\left[y_{i} \mid u\right]=a_{i}(\phi) \mathrm{V}\left(\mu_{i}\right) ; \\
\eta_{i}=g\left(\mu_{i}\right)=x_{i}^{\prime} \beta+z_{i}^{\prime} u,
\end{gathered}
$$

em que: $g\left(\mu_{i}\right)$ é a função de ligação, sendo $x_{i}^{\prime}$ a i-ésima linha da matriz do modelo referente aos efeitos fixos (conhecida); $\beta$ é o vetor dos parâmetros de efeitos fixos (desconhecido); $z_{i}^{\prime}$ é a i-ésima linha da matriz do modelo referente aos efeitos aleatórios (conhecida) e $u$ é o vetor dos efeitos aleatórios (desconhecido).

Para incorporar um aspecto estocástico à variável resposta, o modelo observacional é expresso como desvios aleatórios de $y_{i} \mid u$ em relação a suas médias $\mu_{i}$.

Assim,

$$
y_{i} \mid u=g^{-1}\left(x_{i}^{\prime} \beta+z_{i}^{\prime} u\right)+e_{i},
$$

em que $e_{i}$ é uma variável aleatória com média 0 e $\operatorname{Var}\left[e_{i}\right]=A$, sendo $A$ uma matriz diagonal composta das funções de variâncias $\left(\mathrm{V}\left[\mu_{i}\right]\right)$.

Para completar a especificação do modelo, atribui-se uma distribuição aos efeitos aleatórios,

$$
u \sim f_{U}(u) .
$$

Embora, $u$ possa assumir qualquer distribuição, em geral, assume-se a distribuição normal. Logo,

$$
u \sim \text { i.i.d. } \mathrm{N}(0 ; G), \text { sendo } G=I_{q_{i}} \sigma_{i}^{2} .
$$

Dessa forma, um modelo linear generalizado misto é composto de três partes: primeira, um preditor linear, $\eta=X \beta+Z u$ é usado para modelar a relação entre os efeitos fixos e aleatórios; segunda, uma função de ligação inversa, $\mu_{i}=g^{-1}\left(\eta_{i}\right)$ é usada para modelar a relação entre o preditor linear e a média condicional da característica observada, e terceira, uma função de variância, $\mathrm{V}\left(\mu_{i}\right)$ é usada para modelar a 
variabilidade residual. A variabilidade residual é incorporada na função de variância do modelo linear generalizado misto.

Tem-se que a média marginal de $y_{i}$ é dada por:

$$
\mathrm{E}\left(y_{i}\right)=\mathrm{E}\left[\mathrm{E}\left(y_{i} \mid u\right)\right]=\mathrm{E}\left(\mu_{i}\right)=\mathrm{E}\left[g^{-1}\left(x_{i}^{\prime} \beta+z_{i}^{\prime} u\right)\right],
$$

que, em geral, não pode ser simplificada devido à presença de funções não-lineares em $g^{-1}($.$) .$

A variância marginal de $y_{i}$ é dada por:

$$
\begin{aligned}
\operatorname{Var}\left(y_{i}\right) & =\operatorname{Var}\left[\mathrm{E}\left(y_{i} \mid u\right)\right]+\mathrm{E}\left[\operatorname{Var}\left(y_{i} \mid u\right)\right] \\
& =\operatorname{Var}\left(\mu_{i}\right)+\mathrm{E}\left[a_{i}(\phi) \mathrm{V}\left(\mu_{i}\right)\right] \\
& =\operatorname{Var}\left[g^{-1}\left(x_{i}^{\prime} \beta+z_{i}^{\prime} u\right)\right]+\mathrm{E}\left\{a_{i}(\phi) \mathrm{V}\left[g^{-1}\left(x_{i}^{\prime} \beta+z_{i}^{\prime} u\right)\right]\right\},
\end{aligned}
$$

não sendo possiveis simplificações, sem se fizerem pressuposições especificas a respeito da forma de $g($. ) e/ou a distribuição condicional de $y$.

O uso de efeitos aleatórios introduz uma correlação entre observações que tenham algum efeito em comum. Pressupondo-se independência condicional dos elementos de $y$, tem-se que:

$$
\begin{aligned}
\operatorname{Cov}\left(y_{i}, y_{j}\right) & =\operatorname{Cov}\left[\mathrm{E}\left(y_{i} \mid u\right), \mathrm{E}\left(y_{j} \mid u\right)\right]+\mathrm{E}\left[\operatorname{Cov}\left(y_{i}, y_{j} \mid u\right)\right] \\
& =\operatorname{Cov}\left(\mu_{i}, \mu_{j}\right)+\mathrm{E}(0) \\
& =\operatorname{Cov}\left[g^{-1}\left(x_{i}^{\prime} \beta+z_{i}^{\prime} u\right), g^{-1}\left(x_{j}^{\prime} \beta+z_{j}^{\prime} u\right)\right] .
\end{aligned}
$$

Os estimadores resultantes dependem da função geradora de momentos da variável aleatória (McCulloch \& Searle, 2001).

\subsubsection{Estimação}

De (6), (7) e (8), tem-se que a função de verossimilhança para esse modelo é dada por:

$$
L=\int \prod_{i} f_{Y_{i} \mid U}\left(y_{i} \mid u\right) f_{U}(u) d u=\prod_{i} \int f\left(y_{i}, u\right) d u=\prod_{i=1}^{n} f_{Y}(y),
$$

em que a integração é sobre a distribuição de $u$, de dimensões $q$ x 1 . 
Ao contrário do modelo linear misto clássico, essa função densidade, em geral, não tem uma forma analiticamente tratável, pois envolve integrais de altas dimensões, principalmente se houver muitos efeitos aleatórios a serem estimados (McCulloch \& Searle, 2001). Em casos mais simples, a integração numérica para o cálculo da verossimilhança é direta e, conseqüentemente, a maximização numérica da função de verossimilhança não é difícil, já que o logaritmo da função de verossimilhança é a soma das contribuições independentes de cada agrupamento, que envolve apenas uma integral de dimensão única, que pode ser calculada usando-se técnicas de quadratura.

$\mathrm{Na}$ análise de um modelo linear generalizado misto existem dois problemas que devem ser considerados: primeiro, é freqüentemente dificil especificar uma distribuição particular para os efeitos aleatórios; segundo, a estimação por máxima verossimilhança, baseada na distribuição marginal das observações, envolve integração dos efeitos aleatórios, nos quais, exceto em casos especiais, é numericamente impossível, pois envolvem integrais de alta dimensão (Stiratelli et al., 1984).

Entretanto, vários autores têm sugerido propostas para contornar tais problemas. Dentre elas, as principais maneiras de se avaliar esta densidade são: aproximar a densidade conjunta por uma forma mais numericamente tratável ou integrar numericamente a densidade conjunta. Estes dois enfoques correspondem aos métodos: quase-verossimilhança e integração pelo método da quadratura Gaussiana, respectivamente.

\subsubsection{Método da máxima verossimilhança}

Uma alternativa de estimação é usar um método de integração numérica. Esse método apresenta bons resultados, quando existe um único conjunto de efeitos aleatórios independentes, e tem a vantagem que as estimativas serão de fato de máxima verossimilhança. Entretanto, torna-se impraticável quando existem vários efeitos aleatórios a serem estimados ou quando os efeitos aleatórios são correlacionados (McCulloch \& Searle, 2001). 
Seja $y_{i j}$ a $j$-ésima observação $(j=1, \ldots, k)$ correspondente ao $i$-ésimo nível $(i=1, \ldots, n)$ do efeito aleatório, tal que:

$$
\begin{gathered}
Y_{i j} \mid u \sim \text { indep. } f_{Y_{i j} \mid U}\left(y_{i j} \mid u\right), \\
f_{Y_{i j} \mid U_{i}}\left(y_{i j} \mid u\right)=\exp \left\{\frac{1}{a_{i}(\phi)}\left[y_{i j} \theta_{i j}-b\left(\theta_{i j}\right)\right]+c\left(y_{i j} ; \phi\right)\right\}, \\
\left.\mathrm{E}\left(y_{i j} \mid u\right)\right]=\mu_{i j}, \\
\eta_{i j}=x_{i j}^{\prime} \beta+z_{i j}^{\prime} u, \\
u \sim \text { i.i.d. } \mathrm{N}\left(0 ; \sigma_{u}^{2}\right) .
\end{gathered}
$$

A função de verossimilhança para esse modelo é dada por:

$$
\begin{aligned}
L=\prod_{i, j} \int f\left(y_{i j}, u_{i}\right) d u & =\int \prod_{i, j} f_{Y_{i j} \mid U_{i}}\left(y_{i j} \mid u_{i}\right) f_{U_{i}}\left(u_{i}\right) d u_{i} \\
& =\prod_{i=1}^{n} \int_{-\infty}^{\infty} h_{i}\left(u_{i}\right) \frac{\exp \left\{-\frac{u_{i}^{2}}{2 \sigma_{u}^{2}}\right\}}{\sqrt{2 \pi \sigma_{u}^{2}}} d u_{i},
\end{aligned}
$$

em que: $h_{i}\left(u_{i}\right)=\exp \left\{\sum_{j} \frac{1}{a_{i}(\phi)}\left[y_{i j} \theta_{i j}-b\left(\theta_{i j}\right)\right]+\sum_{j} c\left(y_{i j} ; \phi\right)\right\}$.

Nota-se que a função de verossimilhança é o produto de integrais unidimensionais da forma:

$$
\int_{-\infty}^{\infty} h(u) \frac{\exp \left\{-\frac{u^{2}}{2 \sigma_{u}^{2}}\right\}}{\sqrt{2 \pi \sigma_{u}^{2}}} d u
$$

Fazendo-se uma mudança de variável para:

$$
v=\frac{u}{\sqrt{2 \sigma_{u}^{2}}},
$$

essa integral fica:

$$
\int_{-\infty}^{\infty} h\left(\sqrt{2 \sigma_{u}^{2}} v\right) \frac{\exp \left\{-v^{2}\right\}}{\sqrt{\pi}} d v \equiv \int_{-\infty}^{\infty} h^{*}(v) \exp \left\{-v^{2}\right\} d v
$$


em que: $h^{*}(v)=\frac{h\left(\sqrt{2 \sigma^{2}} v\right)}{\sqrt{\pi}}$.

Integrais, como esta, podem ser avaliadas pelo método da quadratura Gauss-Hermite. Esse método aproxima a integral por uma soma ponderada, da seguinte forma:

$$
\int_{-\infty}^{\infty} h^{*}(v) \exp \left\{-v^{2}\right\} d v=\sum_{i=1}^{d} h^{*}\left(x_{k}\right) w_{k}
$$

sendo os pesos $w_{k}$, e os pontos de avaliação $x_{k}$, escolhidos de tal forma que proporcionem uma aproximação precisa, no caso em que $h^{*}($.) é um polinômio.

Os valores de $x_{k}$ e $w_{k}$ podem ser avaliados em tabelas próprias, por exemplo, em Abramowitz \& Stegum (1964).

\subsection{Equações de verossimilhança}

i) Para os parâmetros de efeitos fixos:

Embora uma solução para as equações de verossimilhança seja numericamente difícil, pode-se escreve-las numa forma mais simples. De (9), tem-se:

$$
\ell=\log f_{Y}(y) \text {. }
$$

Assim,

$$
\begin{aligned}
\frac{\partial \ell}{\partial \beta} & =\frac{\partial}{\partial \beta}\left[\log \int f_{Y \mid u}(y \mid u) f_{U}(u) d u\right] \\
& =\frac{1}{\int f_{Y \mid u}(y \mid u) f_{U}(u) d u}\left[\frac{\partial}{\partial \beta} \int f_{Y \mid u}(y \mid u) f_{U}(u) d u\right] \\
& =\frac{1}{f_{Y}(y)} \int\left[\frac{\partial}{\partial \beta} f_{Y \mid u}(y \mid u)\right] f_{U}(u) d u,
\end{aligned}
$$

pois $f_{U}(u)$ não envolve $\beta$.

Note que, 


$$
\begin{aligned}
\frac{\partial}{\partial \beta} f_{Y \mid u}(y \mid u) & =\frac{1}{f_{Y \mid u}(y \mid u)}\left[\frac{\partial}{\partial \beta} f_{Y \mid u}(y \mid u)\right] f_{Y \mid u}(y \mid u) \\
& =\left[\frac{\partial}{\partial \beta} \log f_{Y \mid u}(y \mid u)\right] f_{Y \mid u}(y \mid u) .
\end{aligned}
$$

Logo,

$$
\begin{aligned}
\frac{\partial \ell}{\partial \beta} & =\frac{1}{f_{Y}(y)} \int\left[\frac{\partial}{\partial \beta} \log f_{Y \mid u}(y \mid u) f_{Y \mid u}(y \mid u)\right] f_{U}(u) d u \\
& =\int\left[\frac{\partial \ell}{\partial \beta} \log f_{Y \mid u}(y \mid u)\right] \frac{f_{Y \mid u}(y \mid u) f_{U}(u) d u}{f_{Y}(y)} \\
& =\int\left[\frac{\partial \ell}{\partial \beta} \log f_{Y \mid u}(y \mid u)\right] f_{U \mid y}(u \mid y) d u .
\end{aligned}
$$

Tem-se, que:

$$
\frac{\partial \ell}{\partial \beta}=X^{\prime} W \Delta(y-\mu)
$$

em que: $W=\operatorname{diag}\left\{W_{i}\right\}=\operatorname{diag}\left[a_{i}(\phi) V\left(\mu_{i}\right) \frac{\partial \eta_{i}}{\partial \mu_{i}}\right]^{2}$ e $\Delta=\operatorname{diag}\left\{\frac{\partial \eta_{i}}{\partial \mu_{i}}\right\}$

Portanto,

$$
\frac{\partial \ell}{\partial \beta}=\int X^{\prime} W^{*}(y-\mu) f_{U \mid y}(u \mid y) d u,
$$

em que $W^{*}=\operatorname{diag}\left[a_{i}(\phi) V\left(\mu_{i}\right) \frac{\partial \eta_{i}}{\partial \mu_{i}}\right]$.

Logo,

$$
\frac{\partial \ell}{\partial \beta}=X^{\prime} y \mathrm{E}\left[W^{*} \mid y\right]-X^{\prime} \mathrm{E}\left[W^{*} \mu \mid y\right] .
$$

Assim, a equação de verossimilhança para $\beta$ é dada por:

$$
X^{\prime} y \mathrm{E}\left[W^{*} \mid y\right]=X^{\prime} \mathrm{E}\left[W^{*} \mu \mid y\right] .
$$


ii) Para os parâmetros de efeitos aleatórios:

Um resultado similar à expressão (11) pode ser encontrado para equações de máxima verossimilhança para os parâmetros referentes à distribuição de $f_{U}(u)$. Seja $\varphi$ denotando os parâmetros de efeitos aleatórios.

De (10), tem-se:

$$
\begin{aligned}
\frac{\partial \ell}{\partial \varphi} & =\frac{1}{f(y)} \frac{\partial f(y)}{\partial \varphi} \\
& =\frac{1}{f(y)} \frac{\partial}{\partial \varphi}\left[\int f(y \mid u) f(u) d u\right] \\
& =\int\left[\frac{\partial}{\partial \varphi} f(y \mid u)\right] \frac{f(u)}{f(y)} d u+\int \frac{f(y \mid u)}{f(u)}\left[\frac{\partial}{\partial \varphi} f(u)\right] d u \\
& =\int \frac{f(y, u)}{f(y)} \frac{1}{f(u)}\left[\frac{\partial}{\partial \varphi} f(u)\right] d u \\
& =\int f_{U \mid y}(u \mid y)\left[\frac{\partial}{\partial \varphi} \log f(u)\right] d u \\
& =\mathrm{E}\left[\frac{\partial}{\partial \varphi} \log f(u) \mid y\right],
\end{aligned}
$$

a qual não pode ser simplificada sem que se especifique uma forma para a distribuição dos efeitos aleatórios.

Dentre os algoritmos utilizados para calcular as estimativas de máxima verossimilhança, destaca-se o algoritmo Maximização da Esperança (EM), descrito por Dempster et al. (1977). Pela facilidade de se imporem restrições às soluções e também pela menor exigência computacional, o uso desse algoritmo é defendido por vários autores, entre eles Henderson (1984 e 1986) e Laird et al. (1987).

O algoritmo EM é um método iterativo para cálculo de estimativas de máxima verossimilhança quando as observações podem ser vista como dados incompletos. Uma vez que cada iteração do algoritmo consiste em um passo de 
esperança (passo E) seguido por um passo de maximização (passo $\mathrm{M}$ ), o algoritmo foi denominado de "Algoritmo EM" (Dempster et al, 1977).

O algoritmo EM toma a seguinte forma:

1) Escolhem-se valores iniciais para $\beta^{(0)}, \theta^{0)}$ e $D^{(0)}$. Estabeleça $m=0$;

2) Calculam-se:

a) $\beta^{(m+1)}$ e $\theta^{(m+1)}$ para maximizar $\mathrm{E}\left[\log f_{Y \mid u}(y \mid u, \beta, \theta) \mid y\right]$;

b) $D^{(m+1)}$ para maximizar $\mathrm{E}\left[\log f_{u}(u \mid \mathrm{D}) \mid y\right]$;

c) Estabeleça $m=m+1$.

3) Se a convergência for atingida, os valores obtidos são as estimativas de máxima verossimilhança, caso contrário, retorne ao passo 2 .

No processo de estimação dos parâmetros, em que o algoritmo EM é utilizado, podem ocorrer algumas dificuldades no processo de integração devido, por exemplo, à quantidade de parâmetros envolvidos. Quando a dimensão da integração envolvida no passo $\mathrm{E}$ do algoritmo EM é um ou dois, técnicas de integração numérica podem ser utilizadas, como por exemplo o método da quadratura Gauss-Hermite. Se as dimensões envolvidas forem de ordem superior a um ou dois, outros métodos devem ser utilizados, tais como o de "Markov chain Monte Carlo" (McCulloch \& Searle, 2001).

\subsubsection{Método da quase-verossimilhança}

Schall (1991) e Breslow \& Clayton (1993), sugerem a utilização de algoritmos de maximização conjunta para obtenção das estimativas dos parâmetros pela maximização do logaritmo da função de quase-verossimilhança conjunta das observações condicionais e dos efeitos aleatórios.

As características da função de quase-verossimilhança, isto é, modelos robustos e menos pressuposições restritivas, têm conduzido a procura de generalizações aplicáveis aos modelos lineares generalizados mistos. A idéia básica é através do uso de uma aproximação Laplace (Wolfinger, 1993), para avaliar integrais de altas dimensões na função de verossimilhança. A aproximação é baseada numa expansão em série de 
Taylor de segunda ordem do logaritmo da função de verossimilhança, e a densidade aproximada será similar a densidade normal.

A forma básica da aproximação de Laplace é dada por:

$$
\ell=\log \int e^{h(u)} d u=h\left(u_{0}\right)+\frac{q}{2} \log 2 \pi-\frac{1}{2} \log \left|-\frac{\partial^{2} h(u)}{\partial u \partial u^{\prime}}\right|,
$$

sendo $u_{0}$ a solução para:

$$
\left.\frac{\partial h(u)}{\partial u}\right|_{u=u_{0}}=0 .
$$

Utiliza-se desse resultado para aproximar o logaritmo da função de verossimilhança do modelo linear generalizado misto via,

$$
\begin{aligned}
\ell & =\log \int f_{Y \mid u} f_{U} d u \\
& =\log \int e^{\log f_{Y_{\mid u}}+\log f_{U}} d u \\
& =\log \int e^{h(u)} d u,
\end{aligned}
$$

em que $h(u)=\log f_{Y \mid u}+\log f_{U}$.

Assumindo-se que $u \sim$ i.i.d. $\mathrm{N}(0 ; G)$, em que $G=\mathrm{I}_{q} \sigma_{u}^{2}$, tem-se:

$$
\log f_{U}=-\frac{1}{2} u^{\prime} G^{-1} u-\frac{q}{2} \log 2 \pi-\frac{1}{2} \log |G| .
$$

Assim, $h(u)$ fica:

$$
h(u)=\log f_{Y \mid u}-\frac{1}{2} u^{\prime} G^{-1} u-\frac{q}{2} \log 2 \pi-\frac{1}{2} \log |G|,
$$

e (12) toma a forma:

$$
\ell=\log f_{Y \mid u_{0}}\left(y \mid u_{0}\right)-\frac{1}{2} u_{0}^{\prime} G^{-1} u_{0}-\frac{1}{2} \log |G|-\frac{1}{2} \log \left|-\frac{\partial^{2} h(u)}{\partial u \partial u^{\prime}}\right| .
$$

A partir de (6), tem-se que $\log f_{Y \mid u}$ é dado por:

$$
\begin{aligned}
\log f_{Y_{i} \mid:} & =\sum_{i} \frac{1}{a_{i}(\phi)}\left[y_{i} \theta_{i}-b\left(\theta_{i}\right)\right]+c\left(y_{i} ; \phi\right) \\
& =\sum_{i} l_{i},
\end{aligned}
$$


sendo $\mathrm{E}\left(y_{i} \mid u_{i}\right)=\mu_{i} \mathrm{e} \eta_{i}=g\left(\mu_{i}\right)=x_{i}^{\prime} \beta+z_{i}^{\prime} u$.

Logo,

$$
h(u)=\sum_{i} l_{i}-\frac{1}{2} u^{\prime} G^{-1} u-\frac{q}{2} \log 2 \pi-\frac{1}{2} \log |G| .
$$

A derivada de $h(u)$ em relação a $u$, é dada por:

$$
\frac{\partial h(u)}{\partial u}=\frac{\partial \sum_{i} l_{i}}{\partial u}-G^{-1} u .
$$

Pela regra da cadeia, tem-se que:

$$
\frac{\partial \sum_{i} l_{i}}{\partial u_{i}}=\sum_{i} \frac{d l_{i}}{d \theta_{i}} \frac{d \theta_{i}}{d \mu_{i}} \frac{d \mu_{i}}{d \eta_{i}} \frac{\partial \eta_{i}}{\partial u_{i}} .
$$

Portanto,

$$
\frac{\partial \sum_{i} l_{i}}{\partial u_{i}}=\sum_{i} \frac{1}{a_{i}(\phi)}\left[y_{i}-b^{\prime}\left(\theta_{i}\right)\right] \frac{1}{\frac{d \mu_{i}}{d \theta_{i}}} \frac{d \mu_{i}}{d \eta_{i}} z_{i}^{\prime} .
$$

Tem-se que:

$$
\mathrm{E}\left(y_{i} \mid u\right)=\mu_{i}=b^{\prime}\left(\theta_{i}\right) \text { e } \operatorname{Var}\left(y_{i} \mid u\right)=a_{i}(\phi) \mathrm{V}\left(\mu_{i}\right)=a_{i}(\phi) \frac{d \mu_{i}}{d \theta_{i}}
$$

Assim,

$$
\frac{d \mu_{i}}{d \theta_{i}}=\mathrm{V}\left(\mu_{i}\right) \text { e } \frac{d \theta_{i}}{d \mu_{i}}=\frac{1}{\mathrm{~V}\left(\mu_{i}\right)}
$$

Logo,

$$
\frac{\partial \sum_{i} l_{i}}{\partial u_{i}}=\sum_{i} \frac{1}{a_{i}(\phi)}\left[y_{i}-\mu_{i}\right] \frac{1}{V\left(\mu_{i}\right)} \frac{d \mu_{i}}{d \eta_{i}} z_{i}^{\prime} .
$$

Tem-se que $a_{i}(\phi)=\frac{\phi}{w_{i}}$, com $\phi>0$, constante, e $w_{i}$ o peso "a priori", geralmente 1.

Portanto, 


$$
\frac{\partial \sum_{i} l_{i}}{\partial u_{i}}=\sum_{i} \frac{w_{i}}{\phi} \frac{\left[y_{i}-\mu_{i}\right]}{\mathrm{V}\left(\mu_{i}\right)} \frac{d \mu_{i}}{d \eta_{i}} z_{i}^{\prime} .
$$

Fazendo-se,

$$
W_{i}=\frac{w_{i}}{\mathrm{~V}\left(\mu_{i}\right)}\left[\frac{d \mu_{i}}{d \eta_{i}}\right]^{2} \text {, }
$$

obtém-se,

$$
\frac{\partial \sum_{i} l_{i}}{\partial u_{i}}=\sum_{i} \frac{1}{\phi} z_{i}^{\prime} W_{i} \frac{d \eta_{i}}{d \mu_{i}}\left(y_{i}-\mu_{i}\right),
$$

que na forma matricial toma a forma:

$$
\frac{\partial \sum_{i} l_{i}}{\partial u_{i}}=\frac{1}{\phi} Z^{\prime} W \Delta(y-\mu),
$$

em que $\Delta=\operatorname{diag}\left(\frac{d \eta_{i}}{d \mu_{i}}\right)$

Portanto,

$$
\frac{\partial h(u)}{\partial u}=\frac{1}{\phi} Z^{\prime} W \Delta(y-\mu)-G^{-1} u .
$$

A segunda derivada de $h(u)$ em relação a $u$ é dada por:

$$
\frac{\partial^{2} h(u)}{\partial u \partial u^{\prime}}=-\frac{1}{\phi} Z^{\prime} W \Delta \frac{\partial \mu}{\partial u^{\prime}}+\frac{1}{\phi} Z^{\prime} \frac{\partial W \Delta}{\partial u^{\prime}}(y-\mu)-G^{-1} .
$$

No caso de funções de ligação canônicas, $W \Delta=\mathrm{I}$, e assim o segundo termo é zero.

Logo,

$$
\begin{aligned}
\frac{\partial^{2} h(u)}{\partial u \partial u^{\prime}} & =-\frac{1}{\phi} Z^{\prime} W \Delta \Delta^{-1} Z+0-G^{-1} \\
& =-\frac{1}{\phi} Z^{\prime} W Z-G^{-1} .
\end{aligned}
$$

Portanto, 


$$
-\frac{\partial^{2} h(u)}{\partial u \partial u^{\prime}}=\frac{1}{\phi} Z^{\prime} W Z+G^{-1}
$$

Substituindo (14) em (13), obtém-se:

$$
\ell=\log f_{Y \mid u_{0}}\left(y \mid u_{0}\right)-\frac{1}{2} u_{0}^{\prime} G^{-1} u_{0}-\frac{1}{2} \log |G|-\frac{1}{2} \log \left|\frac{1}{\phi} Z^{\prime} W Z+G^{-1}\right| .
$$

Derivando-se $\ell$ em relação a $\beta$, obtém-se:

$$
\begin{aligned}
\frac{\partial \ell}{\partial \beta} & =\frac{\partial \log f_{Y \mid u}\left(y \mid u_{0}\right)}{\partial \beta}-\frac{\partial \frac{1}{2} u_{0}^{\prime} G^{-1} u_{0}}{\partial \beta}-\frac{\partial \frac{1}{2} \log |G|}{\partial \beta}-\frac{\partial \frac{1}{2} \log \left|\frac{1}{\phi} Z^{\prime} W Z+G^{-1}\right|}{\partial \beta} \\
& =\frac{1}{\phi} X^{\prime} W \Delta(y-\mu)-\frac{\partial \frac{1}{2} u_{0}^{\prime} G^{-1} u_{0}}{\partial \beta}-\frac{\partial \frac{1}{2} \log |G|}{\partial \beta}-\frac{\partial \frac{1}{2} \log \left|\frac{1}{\phi} Z^{\prime} W Z+G^{-1}\right|}{\partial \beta} \\
& =\frac{1}{\phi} X^{\prime} W \Delta(y-\mu) .
\end{aligned}
$$

E assim, resolvendo-se conjuntamente o sistema de equações:

$$
\begin{gathered}
\frac{1}{\phi} X^{\prime} W \Delta(y-\mu)=0 ; \\
\frac{1}{\phi} Z^{\prime} W \Delta(y-\mu)=G^{-1} u,
\end{gathered}
$$

obtêm-se as estimativas de máxima verossimilhança aproximadas para $\beta$ e $u$.

Breslow \& Clayton (1993) e Wolfinger \& O’Connell (1993) mostraram que as estimativas dos parâmetros referentes aos efeitos fixos e aleatórios de um modelo linear generalizado misto podem ser obtidas através da solução de (15) e (16) via algoritmo escore de Fisher, pela solução iterativa das equações do modelo linear generalizado misto dadas por:

$$
\left[\begin{array}{cc}
X^{\prime} W X & X^{\prime} W Z \\
Z^{\prime} W Z & Z^{\prime} W Z+G^{-1}
\end{array}\right]\left[\begin{array}{l}
\beta \\
u
\end{array}\right]=\left[\begin{array}{c}
X^{\prime} W Y^{*} \\
Z^{\prime} W Y^{*}
\end{array}\right]
$$

em que: 
- Wé uma matriz diagonal com elementos $w_{i}=\frac{\left(\frac{\partial \mu_{i}}{\partial \eta_{i}}\right)^{2}}{V\left(\mu_{i}\right)}$;

- $Y^{*}=\hat{\eta}+\Delta(y-\hat{\mu})$ é a variável dependente ajustada, a qual é uma combinação linear do preditor $\eta$ e da discrepância entre os valores observados e ajustados;

- $\hat{\mu}=E(y)$;

- $\Delta$ é uma matriz diagonal com elementos $\left(\frac{\partial \eta_{i}}{\partial \mu_{i}}\right)$.

Os procedimentos de Breslow \& Clayton (1993) e Wolfinger \& O'Connell (1993) são similares, já que ambos usam as equações de modelos lineares generalizados mistos. Mas, o procedimento de Breslow \& Clayton (1993), o qual chamaram de quase-verossimilhança penalizada ( $\mathrm{PQL}$ ), assume que o parâmetro de dispersão $\phi$ é igual a 1. Os procedimentos de Wolfinger \& O'Connell (1993), os quais chamaram de pseudoverossimilhança (PL) ou pseudoverossimilhança restrita (REPL), assumem que o parâmetro de dispersão $\phi$ é desconhecido. A pseudoverossimilhança (PL) leva a uma estimativa de $\phi$ semelhante à de máxima verossimilhança e a pseudoverossimilhança restrita (REPL) a uma estimativa de $\phi$ semelhante à de máxima verossimilhança restrita.

\subsection{Componentes de variância para dados binários}

Técnicas para a estimação de componentes de variância para dados binários ( 0 's e 1's) ou dados categóricos, são mais raras do que para dados contínuos. A falta de métodos para tais tipos de dados, é devido, em grande parte, pela dificuldade de se especificarem modelos realísticos, e uma vez especificados, a sua intratabilidade computacional. 
No caso contínuo o termo do erro é definido como sendo: $Y-\mathrm{E}(Y \mid u)$ e assim, atribui-se uma distribuição a este como sendo $\mathrm{N}\left(0, \sigma_{e}^{2} I\right)$, e em todos os casos a distribuição tem uma variância constante, independentemente do valor da média de $Y$. Mas, isto não é uma pressuposição verdadeira para o caso de dados discretos (Searle $e t$ al., 1992).

Considerando dados binários, onde $y_{i}$ assumem valores $0 \mathrm{e} 1$, então, a variável aleatória $y_{i}$ tem distribuição de Bernoulli com probabilidade de sucesso $p_{i}=\mathrm{P}\left[y_{i}=1\right]=\mathrm{E}\left(y_{i}\right)$ e variância $\operatorname{Var}\left(y_{i}\right)=p_{i}\left(1-p_{i}\right)=\mathrm{E}\left(y_{i}\right)\left[1-\mathrm{E}\left(y_{i}\right)\right]$. Portanto, essa dependência entre média e variância deve ser incluída no modelo. Assim, um modelo com um componente de erro aditivo, com variância constante, não pode capturar a dependência entre média e variância, e portanto, é inadequado para dados categóricos.

Segundo Searle et al. (1992) os métodos e modelos para estimar os componentes de variância para dados binários, são os seguintes:

i) Método da Análise de Variância (ANAVA)

Se os dados consistem de proporções binomiais, com todos os números de ensaios $(n)$ constante, então, as recomendações usuais são analisar as proporções diretamente (ou transformados para arco seno $\sqrt{y} / n$, sendo $y / n$ proporções) usando o método da análise de variância (ANAVA), assumindo que eles têm uma distribuição aproximadamente normal e são homocedásticos. Entretanto, a análise de proporções pode somente ser recomendada quando as proporções estão no meio do intervalo (por ex. $0,2-0,8)$ e não apresentam problemas de heterocedasticidade.

ii) Modelos Logit-Normal

Nesse caso usa-se uma função de ligação logística e assume-se que os efeitos aleatórios são normalmente distribuídos.

Assim, condicional aos efeitos aleatórios $u$, tem-se que:

$$
\begin{gathered}
Y_{i} \mid u \sim \text { ind. Bernoulli }\left[\mathrm{E}\left(Y_{i} \mid u\right)\right], i=1,2, \ldots, n ; \\
\operatorname{logit}\left[\mathrm{E}\left(Y_{i} \mid u\right)\right]=x_{i}^{\prime} \beta+z_{i}^{\prime} u
\end{gathered}
$$




$$
u \sim \text { i.i.d. } \mathrm{N}(0 ; G), \text { sendo } G=I_{q_{i}} \sigma_{i}^{2},
$$

em que: $x_{i}^{\prime}$ e $z_{i}^{\prime}$ são as i-ésimas linhas de $X$ e $Z$, matrizes do modelo referentes aos efeitos fixos e aleatórios, respectivamente.

A função de verossimilhança nesse caso é dada por:

$$
\int f_{Y \mid p}(y \mid p) \exp \left(-\frac{1}{2} u^{\prime} G^{-1} u\right)|G|^{-\frac{1}{2}} d u
$$

em que: $f_{Y \mid p}(y \mid p)=\prod_{i=1}^{n} \exp \left[y_{i}\left(x_{i}^{\prime} \beta+z_{i}^{\prime} u\right)\right]\left[1+\exp \left(x_{i}^{\prime} \beta+z_{i}^{\prime} u\right)\right]^{-1}$, sendo que a função de verossimilhança pode ser avaliada numericamente através da quadratura Gauss-Hermite, ou também usando métodos de quase-verossimilhança.

iii) Modelos Probit-Normal

Modelos probit-normal são uma classe de modelos bastante similares aos modelos logit-normal, substituindo a função logit pela função probit $\Phi($. ), sendo $\Phi($. ) a função densidade acumulada da distribuição normal padrão.

Assim, tem-se o modelo:

$$
\begin{gathered}
Y_{i} \mid u \sim \text { ind. Bernoulli }\left[\mathrm{E}\left(Y_{i} \mid u\right)\right], i=1,2, \ldots, n ; \\
\mathrm{E}\left(Y_{i} \mid u\right)=\Phi\left(x_{i}^{\prime} \beta+z_{i}^{\prime} u\right) ; \\
u \sim \text { i.i.d. } \mathrm{N}(0 ; G), \text { sendo } G=I_{q_{i}} \sigma_{i}^{2} .
\end{gathered}
$$

A função de verossimilhança é dada por:

$$
\int f_{Y \mid p}(y \mid p) \exp \left(-\frac{1}{2} u^{\prime} G^{-1} u\right) \mid G^{-\frac{1}{2}} d u
$$

em que: $f_{Y \mid p}(y \mid p)=\prod_{i=1}^{n} \Phi\left(x_{i}^{\prime} \beta+z_{i}^{\prime} u\right) y^{y_{i}}\left[1-\Phi\left(x_{i}^{\prime} \beta+z_{i}^{\prime} u\right)\right]^{1-y_{i}}$.

Como no caso do modelo logit-normal, essa função de verossimilhança pode ser avaliada numericamente através da quadratura Gauss-Hermite, ou também usando métodos de quase-verossimilhança. 
McCulloch (1994) considerando um modelo probit-normal para dados binários, apresenta a estimação por máxima verossimilhança dos componentes de variância envolvidos no modelo, usando o algoritmo EM e o método de integração numérica através da quadratura Gauss-Hermite. $O$ autor mostra que o estimador de máxima verossimilhança, para o componente de variância do efeito aleatório $u\left(\sigma_{u}^{2}\right)$, envolvido num modelo linear generalizado misto, é dado por:

$$
\hat{\sigma}_{u}^{2}=\frac{\hat{u} \hat{u}}{q},
$$

sendo que $q$ é o número de níveis do efeito aleatório $u$.

Schall (1991) propôs o seguinte estimador para o componente de variância do efeito aleatório $u\left(\sigma_{u}^{2}\right)$ envolvido num modelo linear generalizado misto, dado por:

$$
\hat{\sigma}_{u}^{2}=\frac{\hat{u}^{\prime} \hat{u}}{q-\operatorname{tr} C_{22}^{-1} / \sigma_{u}^{2}}
$$

e para a variância residual, o estimador:

$$
\hat{\sigma}=\frac{\left(Y^{*}-X \hat{\beta}-Z \hat{u}\right)^{\prime} W\left(Y^{*}-X \hat{\beta}-Z \hat{u}\right)}{N-r(x)-q},
$$

em que: $C_{22}$ é a partição da matriz dos coeficientes das equações do modelo linear generalizado misto, referente aos efeitos aleatórios; $q$ é o número de níveis do efeito aleatório $u ; r(x)$ é o posto da matriz $X ;$ tr é o operador traço matricial.

Vencovsky (1992) apresenta os estimadores para os componentes de variância de freqüências alélicas. O autor admite dados de marcadores colhidos numa estrutura hierárquica, considerando: $r$ populações; $m$ progênies por população; $n$ plantas por progênie e dois genes de um dado loco por planta. A variância de interesse é a da variável indexadora $x$, que vale 1 quando um dado alelo (por ex. $\mathrm{A}_{1}$ ) de um loco está na planta, e 0 quando ausente (por ex: $\mathrm{A}_{2}, \mathrm{~A}_{3}, \ldots$ ). Tendo $g$ locos com $k$ alelos cada um, procede-se as $g k$ análises da variância dessa variável $x$.

O modelo considerado pelo autor é o modelo aleatório, o que pressupõe que todas fontes de variação são obtidas por amostragem no estudo. Assim, a análise de 
cada freqüência alélica $\left(x=1\right.$ se o alelo é $A_{1}$ e $x=0$ se diferente de $\left.A_{1}\right)$ tem esquema de análise de variância conforme Tabela 1.

Tabela 1. Esquema da análise de variância das freqüências de um alelo de um dado loco

\begin{tabular}{lllll}
\hline FV & GL & SQ & QM & E(QM) \\
\hline Populações (P) & $r-1$ & $S_{P}$ & $Q_{P}$ & $\sigma_{G}^{2}+2 \sigma_{I}^{2}+2 n \sigma_{F}^{2}+2 m n \sigma_{P}^{2}$ \\
Famílias (F)/P & $r(m-1)$ & $S_{F}$ & $Q_{F}$ & $\sigma_{G}^{2}+2 \sigma_{I}^{2}+2 n \sigma_{F}^{2}$ \\
Indivíduos (I)/F & $r m(n-1)$ & $S_{I}$ & $Q_{I}$ & $\sigma_{G}^{2}+2 \sigma_{I}^{2}$ \\
Genes (G)/I & $r m n$ & $S_{G}$ & $Q_{G}$ & $\sigma_{G}^{2}$ \\
Total & $2 r m n-1$ & $S_{T}$ & & \\
\hline
\end{tabular}

Nessa análise isolada, tem-se que a variância total da freqüência ( $p$ de $\left.A_{1}\right)$ do alelo em questão é dada por:

$$
\sigma_{T}^{2}=\sigma_{P}^{2}+\sigma_{F}^{2}+\sigma_{I}^{2}+\sigma_{G}^{2}=p(1-p) .
$$

Assim, têm-se as seguintes relações paramétricas:

$$
\begin{gathered}
\sigma_{P}^{2}=p(1-p) \theta_{2} ; \\
\sigma_{F}^{2}=p(1-p)\left(\theta_{1}-\theta_{2}\right) ; \\
\sigma_{I}^{2}=p(1-p)\left(F-\theta_{1}\right) ; \\
\sigma_{G}^{2}=p(1-p)(1-F),
\end{gathered}
$$

em que: $\theta_{2}$ é uma medida do grau de associação ou correlação das freqüências gênicas dentro das populações; $\theta_{1}$ refere-se a quanto os genes estão correlacionados dentro das progênies, é uma medida do grau de parentesco (concestralidade) entre os indivíduos nas progênies; $F$ é o coeficiente de endocruzamento de Wright abrangendo a espécie como um todo, representada, na análise, pelo conjunto de $r$ populações.

Estes parâmetros variam entre 0 e 1. Para obter estimativas desses parâmetros, utiliza-se o método dos momentos.

Logo, tem-se que: 


$$
\begin{gathered}
\hat{\sigma}_{P}^{2}=\frac{1}{2 n m}\left[Q_{P}-Q_{F}\right] ; \\
\hat{\sigma}_{F}^{2}=\frac{1}{2 n}\left[Q_{F}-Q_{I}\right] ; \\
\hat{\sigma}_{I}^{2}=\frac{1}{2}\left[Q_{I}-Q_{C}\right] ; \\
\hat{\sigma}_{G}^{2}=Q_{G} ; \\
\hat{\theta}_{1}=\frac{\hat{\sigma}_{F}^{2}+\hat{\sigma}_{P}^{2}}{\hat{\sigma}_{T}^{2}} ; \\
\hat{\theta}_{2}=\frac{\hat{\sigma}_{P}^{2}}{\hat{\sigma}_{T}^{2}} \\
\hat{\theta}_{1}-\hat{\theta}_{2}=\frac{\hat{\sigma}_{F}^{2}}{\hat{\sigma}_{T}^{2}} ; \\
\hat{F}=\frac{\hat{\sigma}_{I}^{2}+\hat{\sigma}_{F}^{2}+\hat{\sigma}_{P}^{2}}{\hat{\sigma}_{T}^{2}} ; \\
\hat{F}-\hat{\theta}_{1}=\frac{\hat{\sigma}_{I}^{2}}{\hat{\sigma}_{T}^{2}} ; \\
1-\hat{F}=\frac{\hat{\sigma}_{G}^{2}}{\hat{\sigma}_{T}^{2}} .
\end{gathered}
$$

\subsection{Estimação do coeficiente de herdabilidade}

Segundo Pereira Neto (1994), em Estatística Genética, estudam-se certas características nos indivíduos sem descontinuidades naturais, chamadas de "variação" ou "caracteres métricos". O estudo de um caráter métrico centraliza-se em torno da análise de sua variação, porque é em termos de variância que são formuladas as questões primárias da genética. $\mathrm{O}$ valor observado, quando um caráter é medido no indivíduo, é o valor fenotípico desse indivíduo; todas as observações de médias, variâncias e covariâncias devem, claramente, ser baseadas na medida do valor fenotípico. 
Ramalho et al. (2000) afirmam que um dos parâmetros genéticos de maior utilidade para os melhoristas é a estimativa da herdabilidade $\left(h^{2}\right)$. Segundo esses autores, a proporção herdável da variabilidade total é designada de herdabilidade, e é possível estimar dois tipos de herdabilidade: herdabilidade no sentido amplo e herdabilidade no sentido restrito.

A herdabilidade no sentido restrito considera apenas a variância genética aditiva, aquela que é fixada pela seleção, e a herdabilidade no sentido amplo, envolve toda a variância genética.

A herdabilidade permite antever a possibilidade de sucesso com a seleção, uma vez que reflete a proporção da variação fenotípica que pode ser herdada. Em outras palavras, ela mede a confiabilidade do valor fenotípico como indicador do valor reprodutivo (Ramalho et al. , 2000).

\subsubsection{Componentes da herdabilidade}

O fenótipo, a aparência externa de um indivíduo, é o resultado da expressão gênica modificada pelo ambiente. Algebricamente, o fenótipo pode ser expresso por:

$$
P_{i}=\mu+g_{i}+e_{i}+(g e)_{i},
$$

em que: $P_{i}$ é o fenótipo observado; $\mu$ é a média geral; $g_{i}$ é o efeito do genótipo; $e_{i}$ é o efeito do ambiente; $(g e)_{i}$ é o efeito da interação genótipo x ambiente.

O conhecimento da magnitude do efeito do ambiente é importante, uma vez que o melhorista nem sempre pode prever suas variações.

As diferenças nos valores fenotípicos são condicionadas por fatores genéticos e ambientais, e pela interação entre genótipos e ambiente. Assim, o valor fenotípico é variável e seus componentes podem ser determinados por análise de variância, fazendo-se:

$$
\sigma_{p}^{2}=\sigma_{e}^{2}+\sigma_{g}^{2}+\sigma_{g e}^{2}
$$


O componente de variância $\sigma_{e}^{2}$ é uma medida das fontes de variação não controláveis. Esse componente é geralmente denominado erro experimental ou variância de ambiente. $\mathrm{O}$ componente de variância $\sigma_{g e}^{2}$ representa as diferenças entre fenótipos causadas pela interação genótipo $\mathrm{x}$ ambiente. $\mathrm{O}$ componente de variância $\sigma_{g}^{2}$ é causado pelas diferenças genéticas entre os indivíduos.

A variância genotípica $\left(\sigma_{g}^{2}\right)$ pode ser subdividida em variância aditiva $\left(\sigma_{A}^{2}\right)$, variância de dominância $\left(\sigma_{D}^{2}\right)$ e variância epistática $\left(\sigma_{I}^{2}\right)$. Os valores de cada componente de variância podem ser estimados experimentalmente.

No sentido amplo, o coeficiente de herdabilidade $\left(h^{2}\right)$ pode ser definido como a razão da variância genotípica $\left(\sigma_{g}^{2}\right)$ pela variância fenotípica $\left(\sigma_{p}^{2}\right)$, isto é:

$$
h^{2}=\frac{\sigma_{g}^{2}}{\sigma_{p}^{2}} .
$$

No sentido restrito, o coeficiente de herdabilidade $\left(h^{2}\right)$ pode ser definido como a razão da variância aditiva $\left(\sigma_{A}^{2}\right)$ pela variância fenotípica $\left(\sigma_{p}^{2}\right)$ :

$$
h^{2}=\frac{\sigma_{A}^{2}}{\sigma_{p}^{2}} .
$$

A herdabilidade no sentido restrito é mais útil, uma vez que ela quantifica a importância relativa da proporção aditiva da variância genética, que pode ser transmitida para a próxima geração.

A variabilidade varia de acordo com as diversas características agronômicas. Uma das teorias é que as características que se desenvolvem em curto período estariam menos sujeitas ao ambiente e, dessa forma, apresentariam maior herdabilidade do que as sujeitas a maior período.

\subsubsection{Fatores que afetam a estimativa da herdabilidade}

Segundo Borém (2001) as estimativas da herdabilidade variam com: 
i) a característica;

ii) o método de estimação;

iii) a diversidade na população;

iv) o nível de endogamia da população;

v) o tamanho da amostra avaliada;

vi) o número e tipo de ambiente considerados;

vii) a unidade experimental considerada;

viii) a precisão na condução do experimento e da coleta de dados.

As estimativas do coeficiente de herdabilidade são feitas em amostras da população, portanto seu tamanho e representatividade afetam os valores obtidos.

O número de ambientes afeta a estimação da herdabilidade, em razão da interação genótipo $\mathrm{x}$ ambiente.

O coeficiente de herdabilidade pode ser estimado com base em dados de uma única planta, em uma parcela, ou com base na média da parcela.

A condução criteriosa da avaliação da população resulta em menor erro experimental e, por conseguinte, em maior herdabilidade. De igual forma, a precisão na coleta dos dados refletirá na magnitude das estimativas do coeficiente de herdabilidade. Em grandes bancos de dados, como nos de programas de melhoramento, erros grosseiros são comuns.

O coeficiente de herdabilidade estimado com base na média das parcelas pode ser obtido por:

$$
h^{2}=\frac{\sigma_{g}^{2}}{\sigma_{e}^{2} / r l+\sigma_{g e}^{2} / l+\sigma_{g}^{2}},
$$

em que: $r$ é o número de repetições e $l$ o número de ambientes.

Pode-se observar pela expressão acima, que o elevado número de ambientes e de repetições contribui para o aumento das estimativas do coeficiente de herdabilidade, supondo-se que $\sigma_{e}^{2}$ e $\sigma_{g e}^{2}$ sejam constantes. 


\subsubsection{Métodos de estimação do coeficiente de herdabilidade}

A escolha do método de estimação do coeficiente de herdabilidade depende dos recursos genéticos disponíveis e da finalidade da estimativa. Dentre os principais, pode-se citar o método dos componentes de variância. Embora outros métodos estejam descritos na literatura, seu emprego dá-se em situações específicas (Borém, 2001).

A estimação do coeficiente de herdabilidade com base nos componentes de variância é um método que permite ao melhorista a utilização de dados normalmente disponíveis em um programa de melhoramento, como os dados de linhagens de um cruzamento que se encontram em avanços de geração. Esse método permite também que o melhorista estime os componentes de variância por intermédio de um dos delineamentos de acasalamento. Cada delineamento difere quanto ao material genético avaliado, o que determina os tipos de variância que podem ser estimados.

Considerando um experimento envolvendo genótipos avaliados em uma localidade, com repetições (blocos ao acaso), tem-se que o esquema da análise de variância para esse tipo de experimento, segundo Borém (2001), é dado na Tabela 2.

Tabela 2. Esquema da análise de variância de um experimento envolvendo linhagens avaliadas em uma localidade, com repetições

\begin{tabular}{llll}
\hline Fontes de Variação & $\mathrm{GL}$ & $\mathrm{QM}$ & $\mathrm{E}(\mathrm{QM})$ \\
\hline Genótipos (G) & $n_{3}=(g-1)$ & $Q M_{3}$ & $\sigma_{e}^{2}+r \sigma_{g}^{2}$ \\
Repetição (R) & $n_{2}=(r-1)$ & $Q M_{2}$ & $\sigma_{e}^{2}+g \sigma_{r}^{2}$ \\
Gx R (Erro) & $n_{l}=(g-1)(r-1)$ & $Q M_{1}$ & $\sigma_{e}^{2}$ \\
\hline
\end{tabular}

Analisando os componentes de variância pertinentes, chegam-se às seguintes expressões:

$$
\hat{\sigma}_{e}^{2}=Q M_{1},
$$




$$
\hat{\sigma}_{g}^{2}=\frac{Q M_{3}-Q M_{1}}{r}
$$

Assim, podem-se obter as estimativas do coeficiente de herdabilidade, dadas por:

i) com base nas parcelas

$$
h^{2}=\frac{\hat{\sigma}_{g}^{2}}{\hat{\sigma}_{g}^{2}+\hat{\sigma}_{e}^{2}} .
$$

ii) com base na média dos genótipos

$$
h^{2}=\frac{\hat{\sigma}_{g}^{2}}{\hat{\sigma}_{g}^{2}+\hat{\sigma}_{e}^{2} / r} .
$$

Os limites inferior e superior de um intervalo de confiança para o coeficiente de herdabilidade com um coeficiente de confiança de $100(1-\alpha) \%$, são dados (Knapp et al., 1985) por:

$$
L I=\left\{1-\left[\left(\frac{Q M_{1}}{Q M_{2}}\right) F_{1-\frac{\alpha}{2}\left(n_{1} ; n_{2}\right)}\right]^{-1}\right\} \text { e } L S=\left\{1-\left[\left(\frac{Q M_{1}}{Q M_{2}}\right) F_{\frac{\alpha}{2}\left(n_{1} ; n_{2}\right)}\right]^{-1}\right\} .
$$

Considerando agora um experimento envolvendo genótipos, localidades e repetições (blocos ao acaso), o esquema da análise de variância para esse tipo de experimento é apresentado na Tabela 3. 
Tabela 3. Esquema da análise de variância de um experimento envolvendo linhagens, localidades e repetições

\begin{tabular}{llll}
\hline Fontes de Variação & $\mathrm{GL}$ & $\mathrm{QM}$ & $\mathrm{E}(\mathrm{QM})$ \\
\hline Localidade (L) & $(l-1)$ & $Q M_{5}$ & $\sigma_{e}^{2}+g \sigma_{r}^{2}+r g \sigma_{l}^{2}$ \\
Repetição/L & $l(r-1)$ & $Q M_{4}$ & $\sigma_{e}^{2}+g \sigma_{r l}^{2}$ \\
Genótipos (G) & $(g-1)$ & $Q M_{3}$ & $\sigma_{e}^{2}+r \sigma_{g l}^{2}+r l \sigma_{g}^{2}$ \\
Gx L & $(g-1)(l-1)$ & $Q M_{2}$ & $\sigma_{e}^{2}+r \sigma_{g l}^{2}$ \\
Gx R/L (Erro) & $(g-1)(r-1) l$ & $Q M_{1}$ & $\sigma_{e}^{2}$ \\
\hline
\end{tabular}

Analisando os componentes de variância pertinentes, chegam-se às seguintes expressões:

$$
\begin{gathered}
\hat{\sigma}_{e}^{2}=Q M_{1}, \\
\hat{\sigma}_{g}^{2}=\frac{Q M_{3}-Q M_{2}}{l r}, \\
\hat{\sigma}_{g l}^{2}=\frac{Q M_{2}-Q M_{1}}{r}
\end{gathered}
$$

Logo, podem-se obter as estimativas do coeficiente de herdabilidade, dadas por:

i) com base nas parcelas

$$
h^{2}=\frac{\hat{\sigma}_{g}^{2}}{\hat{\sigma}_{g}^{2}+\hat{\sigma}_{g l}^{2}+\hat{\sigma}_{e}^{2}} .
$$

ii) com base na média dos genótipos

$$
h^{2}=\frac{\hat{\sigma}_{g}^{2}}{\hat{\sigma}_{g}^{2}+\hat{\sigma}_{g l}^{2} / l+\hat{\sigma}_{e}^{2} / r l} .
$$




\section{METODOLOGIA}

\subsection{Simulação de dados}

Seja um experimento em blocos ao acaso, cujo modelo linear é dado por:

$$
y_{i j}=\mu+t_{i}+b_{j}+e_{i j}
$$

em que: $y_{i j}$ é o valor observado para o tratamento $i$ no bloco $j ; \mu$ é uma constante inerente a todas as observações (média geral); $t_{i}$ é o efeito do $i$-ésimo tratamento $(i=1$, $2, \ldots ., \mathrm{I}) ; b_{j}$ é o efeito do $j$-ésimo bloco $(j=1,2, \ldots, J) ; e_{i j}$ é o erro aleatório.

Considerando esse modelo, foram feitas simulações de dados de proporções. Foram simulados 100 experimentos, com 20 tratamentos e 10 repetições (blocos) para cada experimento, num total de 200 dados para cada experimento.

Para a geração dos dados, foram utilizadas rotinas do software estatístico SAS (Statistical Analysis System, versão 8.2), através da função aleatória RAMBIN, que exige os parâmetros $n$ e $p$ da distribuição binomial, sendo $n>0$ (inteiro) e $0<p<1$, e também uma semente "SEED" para iniciar o processo, cuja expressão é dada por:

$$
\text { RAMBIN (seed, } n, p \text { ). }
$$

Essa função gera observações de uma variável binomial com média $n p$ e variância $n p(1-p)$.

Os dados foram obtidos atribuindo-se diferentes valores para "SEED" em cada simulação, uma vez que para o mesmo valor inicial, a subrotina RAMBIN gera o mesmo valor. Os valores de $p$ também foram diferentes, e o valor de $n$ foi fixado em 6 . Assim, têm-se médias de tratamentos diferentes para cada experimento.

A seguir foram feitas análises univariadas dos dados para cada experimento simulado, através do PROC UNIVARIATE do SAS, para se verificar se os 
mesmos apresentavam normalidade. Foram constatados que os dados não apresentaram normalidade.

\subsection{Modelo linear misto clássico}

Com os experimentos simulados, conforme descrito em 3.1 , considerando um modelo linear misto clássico, dado por:

$$
y_{i j}=\mu+t_{i}+b_{j}+e_{i j}
$$

em que: $y_{i j}$ é a proporção para o tratamento $i$ no bloco $j ; \mu$ é uma constante inerente a todas as observações (média geral); $t_{i}$ é o efeito do $i$-ésimo tratamento $(i=1,2, \ldots ., 20)$, considerado aleatório, em que $t_{i} \sim$ i.i.d. $\mathrm{N}\left(0, \sigma_{t}^{2}\right) ; b_{j}$ é o efeito do $j$-ésimo bloco $(j=1,2$, $\ldots, 10)$, considerado fixo; $e_{i j}$ é o erro aleatório, em que $e_{i j} \sim$ i.i.d. $\mathrm{N}\left(0, \sigma_{e}^{2}\right)$.

As estimativas para os componentes de variância envolvidas no modelo, foram obtidas pelo método da máxima verossimilhança, por meio do procedimento MIXED do SAS, considerando-se os dados originais (proporções) e transformados, utilizando-se as transformações arco seno $\sqrt{y / n}$ e BOX-COX com $\hat{\lambda}=1,5$.

\subsection{Modelo linear generalizado misto}

Sendo os dados simulados descritos em 3.1, proporções, admitiu-se o modelo binomial, assim, usando a função de ligação logística (canônica), um modelo linear generalizado misto para os dados de proporções, tem preditor linear dado por:

$$
\ln \left(\frac{\pi_{i j}}{1-\pi_{i j}}\right)=\mu+t_{i}+b_{j},
$$

em que: $\pi_{i j}$ é a proporção para o tratamento $i$ no bloco $j ; \quad \mu$ é uma constante inerente à todas às observações (média geral); $t_{i}$ é o efeito do $i$-ésimo tratamento $(i=1,2, \ldots ., 20)$ 
considerado aleatório, em que $t_{i} \sim$ i.i.d. $\mathrm{N}\left(0, \sigma_{t}^{2}\right) ; b_{j}$ é o efeito do $j$-ésimo bloco $(j=1,2, \ldots, 10)$ considerado fixo.

Reescrevendo esse modelo como um modelo estatístico, tem-se:

$$
y_{i j}=\frac{\exp \left(\mu+t_{i}+b_{j}\right)}{1+\exp \left(\mu+t_{i}+b_{j}\right)}+e_{i j},
$$

em que: $y_{i j}$ são proporções e $e_{i j}$ é uma variável aleatória com média 0 e variância $n_{i j} \pi_{i j}\left(1-\pi_{i j}\right)$.

As estimativas dos componentes de variância envolvidas nesse modelo, foram obtidas através dos métodos de estimação: quase-verossimilhança (QV), por meio da macro GLIMMIX do SAS; máxima verossimilhança (MV), através do procedimento NLMIXED do SAS.

Para a verificação da qualidade do ajuste do modelo linear generalizado misto (presença de superdispersão ou subdispersão), estimou-se o parâmetro de dispesão $(\phi)$, baseado na estatística de Pearson $X^{2}$ generalizada (Jфrgensen, 1987), dado por:

$$
\hat{\phi}=\frac{X^{2}}{n-p}=\frac{1}{n-p} \sum_{i=1}^{n} \frac{\left(y_{i}-\hat{\mu}_{i}\right)^{2}}{V\left(\hat{\mu}_{i}\right)},
$$

em que: $V\left(\hat{\mu}_{i}\right)$ é a função de variância estimada sob o modelo que está sendo ajustado, sendo $n$ igual ao número de observações e $p$ igual ao número de parâmetros envolvidos no modelo.

\subsection{Coeficiente de herdabilidade}

Para a estimação dos coeficientes de herdabilidade no sentido amplo, com base nas parcelas, para os experimentos simulados, consideraram-se as estimativas dos componentes de variância envolvidas nos modelos descritos em 3.2 e 3.3, e utilizou-se a expressão dada por:

$$
h^{2}=\frac{\hat{\sigma}_{t}^{2}}{\hat{\sigma}_{t}^{2}+\hat{\sigma}_{e}^{2}} .
$$




\subsection{Comparação dos métodos}

Para comparação das estimativas dos coeficientes de herdabilidade no sentido amplo para os experimentos simulados, considerando as diferentes escalas e métodos de estimação, aplicou-se o teste de Permutação de Fisher (Efron \& Tibshirani, 1993).

O teste de Permutação é uma técnica de reamostragem usada para realizar testes estatísticos. Esse teste é realizado com o objetivo de se calcular o nível de significância $(\alpha)$ para se testar uma hipótese $\mathrm{H}_{0}$ e é descrito a seguir.

Sejam duas amostras aleatórias independentes, $Y=\left(y_{1}, y_{2}, \ldots, y_{n}\right)$ e $Z=\left(z_{1}, z_{2}, \ldots, z_{m}\right)$ extraídas de diferentes distribuições de probabilidade $F$ e $G$, em que:

$$
F \rightarrow Y=\left(y_{1}, y_{2}, \ldots, y_{n}\right) \text { independentemente de } G \rightarrow Z=\left(z_{1}, z_{2}, \ldots, z_{m}\right) .
$$

Tendo observado $Y$ e $Z$, deseja-se testar a hipótese de que não existem diferenças entre $F$ e $G$, ou seja, $\mathrm{H}_{0}: F=G$.

O algoritmo do teste é semelhante ao usado no método Bootstrap, mas as reamostragens são realizadas sem reposição.

Assim, seja $N=n+m$ e $v=\left(y_{1}, y_{2}, \ldots, y_{n}, z_{1}, z_{2}, \ldots, z_{m}\right)$. O vetor $G=\left(g_{1}\right.$, $\left.g_{2}, \ldots, g_{\mathrm{n}}\right)$ é usado para indicar a qual grupo pertencem as observações e contém $n y^{\prime} s$ e $m$ $z$ 's. Logo, existem $\left(\begin{array}{l}N \\ n\end{array}\right)=\frac{N !}{n ! m !}$ possíveis vetores $G$, correspondendo a todas as maneiras possíveis de se agruparem $N$ elementos em dois subconjuntos de tamanho $n$ e $m$. Portanto, sob $\mathrm{H}_{0}: F=G$, não faz diferença qual é a ordem dos elementos de $G$. Esse é o princípio do método.

Como $\left(\begin{array}{l}N \\ n\end{array}\right)=\frac{N !}{n ! m !}$ é, geralmente, um número grande, tomam-se $B$ vetores independentes $G^{*}(1), G^{*}(2), \ldots, G^{*}(B)$, cada um deles formado por $n y^{\prime} s$ e $m z^{\prime} s$, tomados numa ordem aleatória (amostra sem reposição). Para cada amostra calcula-se a estatística do teste, combinando-se $G^{*}(B)$ e $v$, dada por:

$$
\hat{\theta}^{*}(b)=S\left(G^{*}(b), v\right), b=1,2, \ldots, B .
$$


O nível de significância do teste $\left(\alpha_{\text {perm }}\right)$ é dado por:

$$
\hat{\alpha}_{\text {perm }}=\frac{\operatorname{prob}_{\text {perm }}\left\{\hat{\theta}^{*}(b) \geq \hat{\theta}\right\}}{B},
$$

em que $\hat{\theta}=S(G, v)$.

Para aplicação desse teste foi utilizado o procedimento MULTTEST do SAS.

\subsection{Seleção do modelo}

Como critérios de seleção para o melhor modelo ajustado, foram calculados: o viés, que é a média de todas as 100 estimativas do coeficiente de herdabilidade, subtraída do valor de referência; erro padrão para se avaliar a efíciência dos estimadores; valores mínimo e máximo; amplitude. Foi verificado também, qual modelo apresentou valor mais próximo do valor de referência do coefíciente de herdabilidade, obtido a partir dos dados utilizados na simulação.

\subsection{Dados de progênies de Eucalyptus grandis}

Para ilustrar a aplicação da metodologia proposta, e com o objetivo de se fazer uma comparação das estimativas dos parâmetros de efeitos fixos, e efeitos aleatórios preditos, sob o enfoque de modelos lineares mistos clássicos, com os dados originais (proporções) e transformados, utilizando-se as transformações arco seno $\sqrt{y / n}$

e BOX-COX, e também considerando-se um modelo linear generalizado misto com função de ligação logística, através dos métodos de estimação: quase-verossimilhança e máxima verossimilhança, utilizaram-se dados provenientes de um experimento para avaliação de progênies de Eucalypius grandis, aos 5 anos de idade, em que a variável resposta são proporções de plantas sobreviventes.

Os dados experimentais são provenientes de um experimento conduzido pela Empresa Brasileira de Pesquisa Agropecuária (EMBRAPA - FLORESTAS). O 
experimento refere-se a um teste de progênies de Eucalyptus grandis, instalado no delineamento em blocos casualizados, com 6 plantas por parcela e 10 repetições, no espaçamento de $3,0 \times 2,0 \mathrm{~m}$. Foram utilizadas 25 progênies maternas, procedentes de Baldy State Forest 194 - Htherton - QLD, Austrália. O experimento foi instalado na Ripasa S.A. Celulose e Papel, localizada no município de Boa Esperança do Sul, SP, Brasil. Foram feitas avaliações no campo nas idades de 1,3 e 5 anos. As características avaliadas foram: altura total; diâmetro à altura do peito (DAP) e sobrevivência. $\mathrm{Na}$ avaliação da sobrevivência foram atribuídos os valores 1 para as plantas vivas e 0 para as mortas.

Considerando a variável sobrevivência de plantas de Eucalyptus grandis, aos 5 anos de idade, um modelo linear misto clássico é dado por:

$$
y_{i j}=\mu+t_{i}+b_{j}+e_{i j}
$$

em que: $y_{i j}$ é a proporção de plantas sobreviventes para o tratamento $i$ no bloco $j ; \mu$ é uma constante inerente a todas as observações (média geral); $t_{i}$ é o efeito do $i$-ésimo tratamento $(i=1,2, \ldots ., 25)$, considerado aleatório, em que $t_{i} \sim$ i.i.d. $\mathrm{N}\left(0, \sigma_{g}^{2}\right) ; b_{j}$ é o efeito do $j$-ésimo bloco $(j=1,2, \ldots, 10)$, considerado fixo; $e_{i j}$ é o erro aleatório, em que $e_{i j} \sim$ i.i.d. $\mathrm{N}\left(0, \sigma_{e}^{2}\right)$.

Foram ajustados modelos considerando-se os dados originais (proporções) e transformados, utilizando-se as transformações arco seno $\sqrt{y / n}$ e BOX$\operatorname{COX}(\hat{\lambda}=1,3)$. Os modelos foram ajustados através do procedimento MIXED do SAS

Sendo os dados de sobrevivência de plantas de Eucalyptus grandis, aos 5 anos de idade, proporções, e admitindo-se o modelo binomial, um modelo linear generalizado misto com função de ligação logística, para os dados de proporções de plantas sobreviventes, tem preditor linear dado por:

$$
\ln \left(\frac{\pi_{i j}}{1-\pi_{i j}}\right)=\mu+p_{i}+b_{j}
$$

em que: $\pi_{i j}$ é a proporção de plantas sobreviventes para a progênie $i$ no bloco $j ; \mu$ é uma constante inerente à todas às observações (média geral); $p_{i}$ é o efeito da $i$-ésima 
progênie $(i=1,2, \ldots ., 25)$ considerado aleatório, em que $p_{i} \sim \mathrm{N}\left(0, \sigma_{g}^{2}\right) ; b_{j}$ é o efeito do $j$-ésimo bloco $(j=1,2, \ldots, 10)$ considerado fixo.

Reescrevendo esse modelo como um modelo estatístico, tem-se:

$$
y_{i j}=\frac{\exp \left(\mu+p_{i}+b_{j}\right)}{1+\exp \left(\mu+p_{i}+b_{j}\right)}+e_{i j},
$$

em que: $y_{i j}$ são proporções de plantas sobreviventes e $e_{i j}$ é uma variável aleatória com média 0 e variância $n_{i j} \pi_{i j}\left(1-\pi_{i j}\right)$.

Esse modelo foi ajustado considerando-se os métodos de estimação: quase-verossimilhança e máxima verossimilhança, por meio da macro GLIMMIX e através do procedimento NLMIXED do SAS, respectivamente.

Para comparação dos modelos foram estimadas as correlações de Spearman entre os efeitos, com o intuito de se verificar o grau de associação entre os modelos, e também uma análise visual dos resíduos para se verificar qual o modelo mais adequado. 


\section{RESULTADOS E DISCUSSÃO}

\subsection{Dados simulados}

\subsubsection{Modelo linear misto clássico}

De acordo com os experimentos simulados segundo o modelo em blocos ao acaso, admitindo-se um modelo linear misto clássico com blocos fixo, tem-se que as estimativas dos componentes de variância, obtidas pelo método da máxima verossimilhança, para efeitos de tratamentos $\left(\hat{\sigma}_{t}^{2}\right)$ e efeito residual $\left(\hat{\sigma}_{e}^{2}\right)$, considerandose os dados na escala original (proporções) e transformados, utilizando-se as transformações: arco seno $\sqrt{y / n}$ e BOX-COX, são apresentados na Tabela 4.

Tabela 4. Estimativas dos componentes de variância, obtidas pelo método da máxima verossimilhança, para os experimentos simulados

\begin{tabular}{ccccccc}
\hline & \multicolumn{2}{c}{ proporções } & \multicolumn{2}{c}{$\operatorname{arcoseno} \sqrt{y / n}$} & \multicolumn{2}{c}{ BOX-COX } \\
\cline { 2 - 7 } $\operatorname{Exp}$ & $\hat{\sigma}_{t}^{2}$ & $\hat{\sigma}_{e}^{2}$ & $\hat{\sigma}_{t}^{2}$ & $\hat{\sigma}_{e}^{2}$ & $\hat{\sigma}_{t}^{2}$ & $\hat{\sigma}_{e}^{2}$ \\
\hline 1 & 0,0096 & 0,0253 & 70,2761 & 186,8000 & 0,0154 & 0,0397 \\
2 & 0,0110 & 0,0287 & 79,3710 & 202,8300 & 0,0171 & 0,0440 \\
3 & 0,0091 & 0,0382 & 58,6364 & 247,0100 & 0,0129 & 0,0518 \\
4 & 0,0080 & 0,0276 & 63,3873 & 189,8600 & 0,0130 & 0,0420 \\
5 & 0,0108 & 0,0284 & 67,5857 & 203,7400 & 0,0160 & 0,0424 \\
6 & 0,0090 & 0,0268 & 70,5772 & 202,8100 & 0,0145 & 0,0424
\end{tabular}


Tabela 4. Estimativas dos componentes de variância, obtidas pelo método da máxima verossimilhança, para os experimentos simulados

\begin{tabular}{ccccccc}
\hline & \multicolumn{2}{c}{ proporções } & \multicolumn{2}{c}{ arco seno $\sqrt{y / n}$} & \multicolumn{2}{c}{ BOX-COX } \\
\cline { 2 - 7 } Exp & $\hat{\sigma}_{t}^{2}$ & $\hat{\sigma}_{e}^{2}$ & $\hat{\sigma}_{t}^{2}$ & $\hat{\sigma}_{e}^{2}$ & $\hat{\sigma}_{t}^{2}$ & $\hat{\sigma}_{e}^{2}$ \\
\hline 7 & 0,0157 & 0,0300 & 114,9200 & 207,0200 & 0,0239 & 0,0440 \\
8 & 0,0093 & 0,0306 & 70,0916 & 229,4900 & 0,0144 & 0,0441 \\
9 & 0,0103 & 0,0332 & 62,2659 & 223,6500 & 0,0152 & 0,0487 \\
10 & 0,0045 & 0,0314 & 32,3744 & 207,8300 & 0,0068 & 0,0464 \\
11 & 0,0119 & 0,0310 & 77,1839 & 227,4500 & 0,0172 & 0,0444 \\
12 & 0,0079 & 0,0325 & 53,7795 & 233,5500 & 0,0123 & 0,0499 \\
13 & 0,0090 & 0,0344 & 72,2934 & 240,6500 & 0,0145 & 0,0490 \\
14 & 0,0082 & 0,0252 & 56,6781 & 179,9300 & 0,0128 & 0,0394 \\
15 & 0,0084 & 0,0310 & 54,7474 & 215,6500 & 0,0125 & 0,0461 \\
16 & 0,0109 & 0,0299 & 79,1790 & 189,9800 & 0,0152 & 0,0429 \\
17 & 0,0121 & 0,0266 & 77,8530 & 187,8600 & 0,0178 & 0,0370 \\
18 & 0,0052 & 0,0314 & 36,0947 & 238,2500 & 0,0079 & 0,0477 \\
19 & 0,0072 & 0,0335 & 54,0251 & 222,1700 & 0,0113 & 0,0485 \\
20 & 0,0107 & 0,0366 & 82,0190 & 253,9600 & 0,0165 & 0,0528 \\
21 & 0,0088 & 0,0331 & 63,2766 & 214,1200 & 0,0133 & 0,0470 \\
22 & 0,0093 & 0,0289 & 50,3651 & 209,2600 & 0,0136 & 0,0436 \\
23 & 0,0081 & 0,0332 & 56,9467 & 213,9500 & 0,0123 & 0,0482 \\
24 & 0,0129 & 0,0313 & 86,6855 & 215,7200 & 0,0193 & 0,0458 \\
25 & 0,0070 & 0,0269 & 43,7961 & 192,1500 & 0,0104 & 0,0413 \\
26 & 0,0093 & 0,0263 & 60,6629 & 177,7200 & 0,0141 & 0,0401 \\
27 & 0,0137 & 0,0336 & 94,5003 & 231,1600 & 0,0203 & 0,0501 \\
28 & 0,0104 & 0,0275 & 69,4663 & 192,5200 & 0,0151 & 0,0398 \\
29 & 0,0085 & 0,0283 & 67,2051 & 194,5800 & 0,0136 & 0,0429 \\
30 & 0,0062 & 0,0366 & 36,3221 & 254,1200 & 0,0086 & 0,0509
\end{tabular}


Tabela 4. Estimativas dos componentes de variância, obtidas pelo método da máxima verossimilhança, para os experimentos simulados

\begin{tabular}{|c|c|c|c|c|c|c|}
\hline \multirow[b]{2}{*}{ Exp } & \multicolumn{2}{|c|}{ proporções } & \multicolumn{2}{|c|}{$\operatorname{arcoseno} \sqrt{y / n}$} & \multicolumn{2}{|c|}{ BOX-COX } \\
\hline & $\hat{\sigma}_{t}^{2}$ & $\hat{\sigma}_{e}^{2}$ & $\hat{\sigma}_{t}^{2}$ & $\overline{\hat{\sigma}_{e}^{2}}$ & $\hat{\sigma}_{t}^{2}$ & $\hat{\sigma}_{e}^{2}$ \\
\hline 31 & 0,0092 & 0,0268 & 70,5793 & 192,5200 & 0,0142 & 0,0368 \\
\hline 32 & 0,0096 & 0,0283 & 57,7778 & 214,3900 & 0,0147 & 0,0441 \\
\hline 33 & 0,0092 & 0,0290 & 56,1431 & 212,8200 & 0,0138 & 0,0433 \\
\hline 34 & 0,0107 & 0,0282 & 61,4746 & 195,0700 & 0,0154 & 0,0419 \\
\hline 35 & 0,0184 & 0,0310 & 119,9200 & 208,6800 & 0,0270 & 0,0452 \\
\hline 36 & 0,0142 & 0,0305 & 86,2476 & 225,8800 & 0,0206 & 0,0463 \\
\hline 37 & 0,0077 & 0,0304 & 54,6990 & 197,8900 & 0,0122 & 0,0446 \\
\hline 38 & 0,0080 & 0,0242 & 45,9976 & 185,0000 & 0,0119 & 0,0381 \\
\hline 39 & 0,0122 & 0,0279 & 75,3246 & 179,8300 & 0,0182 & 0,0402 \\
\hline 40 & 0,0105 & 0,0392 & 56,4449 & 260,9300 & 0,0142 & 0,0546 \\
\hline 41 & 0,0144 & 0,0291 & 99,9565 & 187,9800 & 0,0212 & 0,0420 \\
\hline 42 & 0,0125 & 0,0223 & 89,3593 & 154,2300 & 0,0195 & 0,0337 \\
\hline 43 & 0,0096 & 0,0244 & 73,4919 & 188,8500 & 0,0156 & 0,0388 \\
\hline 44 & 0,0076 & 0,0301 & 59,9081 & 221,1400 & 0,0123 & 0,0463 \\
\hline 45 & 0,0112 & 0,0294 & 71,0521 & 211,2300 & 0,0156 & 0,0411 \\
\hline 46 & 0,0070 & 0,0288 & 52,8668 & 184,0400 & 0,0107 & 0,0425 \\
\hline 47 & 0,0078 & 0,0336 & 52,9310 & 259,4100 & 0,0115 & 0,0506 \\
\hline 48 & 0,0074 & 0,0330 & 56,1957 & 219,4000 & 0,0110 & 0,0453 \\
\hline 49 & 0,0094 & 0,0303 & 54,0106 & 224,8800 & 0,0131 & 0,0474 \\
\hline 50 & 0,0123 & 0,0271 & 77,9893 & 184,2900 & 0,0181 & 0,0405 \\
\hline 51 & 0,0097 & 0,0307 & 61,8570 & 193,8900 & 0,0142 & 0,0440 \\
\hline 52 & 0,0093 & 0,0302 & 55,2884 & 213,5500 & 0,0137 & 0,0462 \\
\hline 53 & 0,0048 & 0,0256 & 34,7003 & 186,4100 & 0,0076 & 0,0400 \\
\hline 54 & 0,0075 & 0,0295 & 45,8430 & 207,6400 & 0,0112 & 0,0449 \\
\hline
\end{tabular}


Tabela 4. Estimativas dos componentes de variância, obtidas pelo método da máxima verossimilhança, para os experimentos simulados

\begin{tabular}{|c|c|c|c|c|c|c|}
\hline \multirow[b]{2}{*}{$\operatorname{Exp}$} & \multicolumn{2}{|c|}{ proporções } & \multicolumn{2}{|c|}{$\operatorname{arcoseno} \sqrt{y / n}$} & \multicolumn{2}{|c|}{ BOX-COX } \\
\hline & $\hat{\sigma}_{t}^{2}$ & $\hat{\sigma}_{e}^{2}$ & $\hat{\sigma}_{t}^{2}$ & $\hat{\sigma}_{e}^{2}$ & $\hat{\sigma}_{t}^{2}$ & $\hat{\sigma}_{e}^{2}$ \\
\hline 55 & 0,0081 & 0,0298 & 54,4598 & 216,4600 & 0,0125 & 0,0453 \\
\hline 56 & 0,0088 & 0,0234 & 71,9712 & 171,1300 & 0,0142 & 0,0361 \\
\hline 57 & 0,0092 & 0,0237 & 70,8120 & 168,9900 & 0,0146 & 0,0368 \\
\hline 58 & 0,0084 & 0,0334 & 64,6404 & 229,1900 & 0,0131 & 0,0503 \\
\hline 59 & 0,0094 & 0,0295 & 70,0328 & 206,5000 & 0,0142 & 0,0430 \\
\hline 60 & 0,0102 & 0,0308 & 67,4761 & 233,5800 & 0,0153 & 0,0462 \\
\hline 61 & 0,0077 & 0,0301 & 50,9810 & 201,0800 & 0,0111 & 0,0442 \\
\hline 62 & 0,0044 & 0,0294 & 25,0062 & 196,9100 & 0,0066 & 0,0450 \\
\hline 63 & 0,0103 & 0,0277 & 67,9178 & 197,8400 & 0,0156 & 0,0407 \\
\hline 64 & 0,0065 & 0,0299 & 42,8800 & 211,9500 & 0,0098 & 0,0461 \\
\hline 65 & 0,0074 & 0,0268 & 52,6105 & 199,7400 & 0,0118 & 0,0420 \\
\hline 66 & 0,0069 & 0,0277 & 55,8984 & 196,4600 & 0,0115 & 0,0432 \\
\hline 67 & 0,0095 & 0,0315 & 67,2707 & 207,3800 & 0,0143 & 0,0456 \\
\hline 68 & 0,0132 & 0,0308 & 87,8787 & 210,6100 & 0,0195 & 0,0453 \\
\hline 69 & 0,0097 & 0,0295 & 71,4915 & 213,8700 & 0,0145 & 0,0431 \\
\hline 70 & 0,0082 & 0,0310 & 69,5132 & 220,1500 & 0,0133 & 0,0465 \\
\hline 71 & 0,0054 & 0,0304 & 39,0895 & 226,4900 & 0,0080 & 0,0472 \\
\hline 72 & 0,0078 & 0,0309 & 51,6049 & 219,0800 & 0,0121 & 0,0452 \\
\hline 73 & 0,0104 & 0,0261 & 71,8868 & 184,5600 & 0,0162 & 0,0397 \\
\hline 74 & 0,0065 & 0,0290 & 40,1195 & 196,6400 & 0,0093 & 0,0434 \\
\hline 75 & 0,0099 & 0,0273 & 69,7149 & 179,2300 & 0,0154 & 0,0403 \\
\hline 76 & 0,0099 & 0,0289 & 64,9640 & 200,2900 & 0,0148 & 0,0437 \\
\hline 77 & 0,0099 & 0,0282 & 79,5997 & 196,1100 & 0,0153 & 0,0422 \\
\hline 78 & 0,0117 & 0,0294 & 72,0619 & 200,9900 & 0,0173 & 0,0434 \\
\hline
\end{tabular}


Tabela 4. Estimativas dos componentes de variância, obtidas pelo método da máxima verossimilhança, para os experimentos simulados

\begin{tabular}{ccccccc}
\hline & \multicolumn{2}{c}{ proporções } & \multicolumn{2}{c}{ arco seno $\sqrt{y} / n$} & \multicolumn{2}{c}{ BOX-COX } \\
\cline { 2 - 7 } Exp & $\hat{\sigma}_{t}^{2}$ & $\hat{\sigma}_{e}^{2}$ & $\hat{\sigma}_{t}^{2}$ & $\hat{\sigma}_{e}^{2}$ & $\hat{\sigma}_{t}^{2}$ & $\hat{\sigma}_{e}^{2}$ \\
\hline 79 & 0,0089 & 0,0263 & 79,6939 & 183,8200 & 0,0151 & 0,0404 \\
80 & 0,0121 & 0,0282 & 85,3655 & 194,4800 & 0,0184 & 0,0403 \\
81 & 0,0124 & 0,0349 & 90,1606 & 225,2700 & 0,0190 & 0,0507 \\
82 & 0,0080 & 0,0239 & 52,0702 & 158,8500 & 0,0122 & 0,0358 \\
83 & 0,0118 & 0,0307 & 90,7705 & 189,3300 & 0,0185 & 0,0437 \\
84 & 0,0081 & 0,0279 & 66,8009 & 186,0500 & 0,0134 & 0,0424 \\
85 & 0,0073 & 0,0339 & 50,5478 & 223,0500 & 0,0115 & 0,0479 \\
86 & 0,0109 & 0,0275 & 63,1456 & 186,7000 & 0,0154 & 0,0411 \\
87 & 0,0125 & 0,0245 & 85,0095 & 178,3500 & 0,0191 & 0,0376 \\
88 & 0,0076 & 0,0333 & 51,9660 & 236,0100 & 0,0113 & 0,0509 \\
89 & 0,0110 & 0,0309 & 73,0132 & 211,7100 & 0,0163 & 0,0461 \\
90 & 0,0087 & 0,0304 & 68,5683 & 224,9700 & 0,0136 & 0,0447 \\
91 & 0,0089 & 0,0302 & 52,8309 & 215,1400 & 0,0125 & 0,0452 \\
92 & 0,0099 & 0,0317 & 63,1770 & 220,2600 & 0,0141 & 0,0471 \\
93 & 0,0068 & 0,0257 & 47,7715 & 183,4900 & 0,0106 & 0,0387 \\
94 & 0,0102 & 0,0259 & 71,9244 & 202,9900 & 0,0157 & 0,0396 \\
95 & 0,0092 & 0,0295 & 64,6338 & 193,5300 & 0,0140 & 0,0436 \\
96 & 0,0130 & 0,0273 & 92,6786 & 200,7300 & 0,0195 & 0,0418 \\
97 & 0,0091 & 0,0262 & 65,1222 & 184,5100 & 0,0141 & 0,0398 \\
98 & 0,0072 & 0,0260 & 44,2767 & 189,5600 & 0,0111 & 0,0406 \\
99 & 0,0073 & 0,0288 & 50,5095 & 198,9300 & 0,0113 & 0,0440 \\
100 & 0,0099 & 0,0273 & 62,3912 & 184,8600 & 0,0148 & 0,0411 \\
\hline & & & & & &
\end{tabular}




\subsubsection{Modelo linear generalizado misto}

A partir dos dados simulados conforme o modelo em blocos ao acaso, admitindo-se um modelo linear generalizado misto, com blocos fixo e função de ligação logística, tem-se que as estimativas dos componentes de variância para efeitos de tratamentos $\left(\hat{\sigma}_{t}^{2}\right)$ e efeito residual $\left(\hat{\sigma}_{e}^{2}\right)$, bem como as estimativas do parâmetro de dispersão $(\hat{\phi})$, obtidas pelos métodos da quase-verossimilhança (QV) e máxima verossimilhança (MV) são apresentados na Tabela 5.

Tabela 5. Estimativas dos componentes de variância e do parâmetro de dispersão, obtidas pelo método da quase-verossimilhança (QV) e máxima verossimilhança (MV), para os experimentos simulados

\begin{tabular}{ccccccc}
\hline & \multicolumn{3}{c}{ QV } & & \multicolumn{3}{c}{ MV } \\
\cline { 2 - 7 } Exp & $\hat{\sigma}_{t}^{2}$ & $\hat{\sigma}_{e}^{2}$ & $\hat{\phi}$ & $\hat{\sigma}_{i}^{2}$ & $\hat{\sigma}_{e}^{2}$ & $\hat{\phi}$ \\
\hline 1 & 0,5756 & 1,0770 & 0,9681 & 0,5783 & 1,0745 & 0,8908 \\
2 & 0,5907 & 1,0922 & 0,9613 & 0,5941 & 1,0899 & 0,9858 \\
3 & 0,5624 & 1,1694 & 0,9736 & 0,5643 & 1,1672 & 1,2325 \\
4 & 0,5588 & 1,1205 & 0,9675 & 0,5605 & 1,1182 & 0,9689 \\
5 & 0,5759 & 1,1174 & 0,9693 & 0,5785 & 1,1150 & 0,9555 \\
6 & 0,5820 & 1,0309 & 0,9527 & 0,5847 & 1,0284 & 1,0011 \\
7 & 0,6243 & 1,1119 & 0,9688 & 0,6299 & 1,1096 & 1,0603 \\
8 & 0,5746 & 1,0502 & 0,9636 & 0,5772 & 1,0473 & 1,1079 \\
9 & 0,5728 & 1,1201 & 0,9692 & 0,5752 & 1,1177 & 1,1245 \\
10 & 0,5348 & 1,1190 & 0,9722 & 0,5354 & 1,1161 & 1,1118 \\
11 & 0,5930 & 1,0543 & 0,9612 & 0,5965 & 1,0517 & 1,0611 \\
12 & 0,5634 & 1,1374 & 0,9696 & 0,5653 & 1,1348 & 1,1008 \\
13 & 0,5820 & 1,1040 & 0,9609 & 0,5848 & 1,1019 & 1,1376 \\
14 & 0,5549 & 1,1246 & 0,9726 & 0,5565 & 1,1220 & 0,8868 \\
15 & 0,5656 & 1,0993 & 0,9688 & 0,5677 & 1,0966 & 1,0509
\end{tabular}


Tabela 5. Estimativas dos componentes de variância e do parâmetro de dispersão, obtidas pelo método da quase-verossimilhança (QV) e máxima verossimilhança (MV), para os experimentos simulados

\begin{tabular}{|c|c|c|c|c|c|c|}
\hline \multirow[b]{2}{*}{ Exp } & \multicolumn{3}{|c|}{ QV } & \multicolumn{3}{|c|}{ MV } \\
\hline & $\hat{\sigma}_{t}^{2}$ & $\hat{\sigma}_{e}^{2}$ & $\hat{\phi}$ & $\hat{\sigma}_{t}^{2}$ & $\overline{\hat{\sigma}_{e}^{2}}$ & $\hat{\phi}$ \\
\hline 16 & 0,5872 & 1,1635 & 0,9687 & 0,5835 & 1,1615 & 0,9542 \\
\hline 17 & 0,5914 & 1,0673 & 0,9597 & 0,5947 & 1,0649 & 0,9074 \\
\hline 18 & 0,5474 & 1,0361 & 0,9754 & 0,5488 & 1,0326 & 1,1497 \\
\hline 19 & 0,5531 & 1,1781 & 0,9681 & 0,5544 & 1,1762 & 1,0808 \\
\hline 20 & 0,5947 & 1,1015 & 0,9636 & 0,5985 & 1,0993 & 1,2132 \\
\hline 21 & 0,5656 & 1,1294 & 0,9684 & 0,5676 & 1,1269 & 1,0982 \\
\hline 22 & 0,5653 & 1,1149 & 0,9714 & 0,5685 & 1,1125 & 0,9620 \\
\hline 23 & 0,5512 & 1,1862 & 0,9699 & 0,5525 & 1,1842 & 1,0968 \\
\hline 24 & 0,5891 & 1,1389 & 0,9677 & 0,5924 & 1,1371 & 1,0508 \\
\hline 25 & 0,5456 & 1,1517 & 0,9744 & 0,5468 & 1,1492 & 0,9039 \\
\hline 26 & 0,5623 & 1,1069 & 0,9655 & 0,5641 & 1,1046 & 0,9043 \\
\hline 27 & 0,6075 & 1,1105 & 0,9611 & 0,6118 & 1,1087 & 1,1288 \\
\hline 28 & 0,5763 & 1,0622 & 0,9620 & 0,5788 & 1,0597 & 0,9786 \\
\hline 29 & 0,5670 & 1,1219 & 0,9714 & 0,5694 & 1,1195 & 0,9840 \\
\hline 30 & 0,5462 & 1,1376 & 0,9798 & 0,5476 & 1,1348 & 1,1914 \\
\hline 31 & 0,5734 & 1,0808 & 0,9595 & 0,5757 & 1,0785 & 0,9164 \\
\hline 32 & 0,5721 & 1,0776 & 0,9708 & 0,5747 & 1,0750 & 1,0049 \\
\hline 33 & 0,5709 & 1,1076 & 0,9700 & 0,5733 & 1,1051 & 0,9730 \\
\hline 34 & 0,5683 & 1,1151 & 0,9697 & 0,5705 & 1,1127 & 0,9663 \\
\hline 35 & 0,6288 & 1,1299 & 0,9610 & 0,6341 & 1,1283 & 1,0361 \\
\hline 36 & 0,6017 & 1,1042 & 0,9674 & 0,6058 & 1,1021 & 1,0371 \\
\hline 37 & 0,5554 & 1,1703 & 0,9685 & 0,5569 & 1,1683 & 1,0228 \\
\hline 38 & 0,5555 & 1,0727 & 0,9683 & 0,5571 & 1,0699 & 0,8798 \\
\hline 39 & 0,5797 & 1,1432 & 0,9681 & 0,5824 & 1,1413 & 0,9311 \\
\hline
\end{tabular}


Tabela 5. Estimativas dos componentes de variância e do parâmetro de dispersão, obtidas pelo método da quase-verossimilhança (QV) e máxima verossimilhança $(\mathrm{MV})$, para os experimentos simulados

\begin{tabular}{|c|c|c|c|c|c|c|}
\hline \multirow[b]{2}{*}{ Exp } & \multicolumn{3}{|c|}{ QV } & \multicolumn{3}{|c|}{ MV } \\
\hline & $\hat{\sigma}_{t}^{2}$ & $\hat{\sigma}_{e}^{2}$ & $\hat{\phi}$ & $\hat{\sigma}_{t}^{2}$ & $\hat{\sigma}_{e}^{2}$ & $\hat{\phi}$ \\
\hline 40 & 0,5706 & 1,1434 & 0,9682 & 0,5727 & 1,1412 & 1,2631 \\
\hline 41 & 0,6108 & 1,1036 & 0,9603 & 0,6153 & 1,1016 & 0,9982 \\
\hline 42 & 0,5979 & 1,0715 & 0,9637 & 0,6019 & 1,0691 & 0,7880 \\
\hline 43 & 0,5910 & 1,0232 & 0,9511 & 0,5941 & 1,0207 & 0,9033 \\
\hline 44 & 0,5661 & 1,0715 & 0,9598 & 0,5681 & 1,0690 & 1,0809 \\
\hline 45 & 0,5743 & 1,0879 & 0,9716 & 0,5769 & 1,0853 & 0,9987 \\
\hline 46 & 0,5513 & 1,1645 & 0,9681 & 0,5525 & 1,1623 & 0,9469 \\
\hline 47 & 0,5610 & 1,0830 & 0,9733 & 0,5630 & 1,0802 & 1,1459 \\
\hline 48 & 0,5586 & 1,1464 & 0,9694 & 0,5602 & 1,1435 & 1,0840 \\
\hline 49 & 0,5621 & 1,1182 & 0,9745 & 0,5641 & 1,1157 & 1,0440 \\
\hline 50 & 0,5808 & 1,1562 & 0,9671 & 0,5836 & 1,1543 & 0,9120 \\
\hline 51 & 0,5658 & 1,1403 & 0,9700 & 0,5679 & 1,1380 & 1,0276 \\
\hline 52 & 0,5636 & 1,1336 & 0,9773 & 0,5658 & 1,1311 & 1,0444 \\
\hline 53 & 0,5319 & 1,1213 & 0,9708 & 0,5323 & 1,1185 & 0,9299 \\
\hline 54 & 0,5569 & 1,1261 & 0,9663 & 0,5584 & 1,1238 & 0,9690 \\
\hline 55 & 0,5659 & 1,1037 & 0,9635 & 0,5679 & 1,1015 & 1,0188 \\
\hline 56 & 0,5780 & 1,0471 & 0,9474 & 0,5800 & 1,0446 & 0,8540 \\
\hline 57 & 0,5767 & 1,0564 & 0,9592 & 0,5792 & 1,0539 & 0,8691 \\
\hline 58 & 0,5720 & 1,1289 & 0,9551 & 0,5739 & 1,1271 & 1,1279 \\
\hline 59 & 0,5628 & 1,1477 & 0,9699 & 0,5647 & 1,1454 & 1,0052 \\
\hline 60 & 0,5868 & 1,0726 & 0,9604 & 0,5899 & 1,0702 & 1,0650 \\
\hline 61 & 0,5514 & 1,1496 & 0,9722 & 0,5528 & 1,1471 & 0,9882 \\
\hline 62 & 0,5251 & 1,2114 & 0,9817 & 0,5255 & 1,2091 & 0,9732 \\
\hline 63 & 0,5711 & 1,0969 & 0,9700 & 0,5735 & 1,0942 & 0,9786 \\
\hline
\end{tabular}


Tabela 5. Estimativas dos componentes de variância e do parâmetro de dispersão, obtidas pelo método da quase-verossimilhança (QV) e máxima verossimilhança (MV), para os experimentos simulados

\begin{tabular}{|c|c|c|c|c|c|c|}
\hline \multirow[b]{2}{*}{ Exp } & \multicolumn{3}{|c|}{ QV } & \multicolumn{3}{|c|}{ MV } \\
\hline & $\hat{\sigma}_{t}^{2}$ & $\hat{\sigma}_{e}^{2}$ & $\hat{\phi}$ & $\hat{\sigma}_{t}^{2}$ & $\hat{\sigma}_{e}^{2}$ & $\hat{\phi}$ \\
\hline 64 & 0,5482 & 1,1338 & 0,9730 & 0,5495 & 1,1313 & 1,0147 \\
\hline 65 & 0,5558 & 1,0976 & 0,9644 & 0,5573 & 1,0951 & 0,9443 \\
\hline 66 & 0,5492 & 1,1456 & 0,9662 & 0,5503 & 1,1433 & 0,9850 \\
\hline 67 & 0,5611 & 1,1794 & 0,9731 & 0,5629 & 1,1773 & 1,0307 \\
\hline 68 & 0,6068 & 1,0977 & 0,9646 & 0,6113 & 1,0955 & 1,0210 \\
\hline 69 & 0,5748 & 1,0561 & 0,9687 & 0,5775 & 1,0532 & 1,0446 \\
\hline 70 & 0,5743 & 1,1010 & 0,9600 & 0,5766 & 1,0988 & 1,0847 \\
\hline 71 & 0,5438 & 1,0797 & 0,9797 & 0,5451 & 1,0765 & 1,0671 \\
\hline 72 & 0,5610 & 1,0969 & 0,9684 & 0,5629 & 1,0942 & 1,0540 \\
\hline 73 & 0,5769 & 1,0981 & 0,9657 & 0,5795 & 1,0958 & 0,9414 \\
\hline 74 & 0,5441 & 1,1385 & 0,9736 & 0,5451 & 1,1358 & 0,9840 \\
\hline 75 & 0,5690 & 1,1368 & 0,9605 & 0,5708 & 1,1345 & 0,9291 \\
\hline 76 & 0,5636 & 1,1400 & 0,9707 & 0,5655 & 1,1379 & 0,9975 \\
\hline 77 & 0,5747 & 1,1463 & 0,9592 & 0,5769 & 1,1444 & 0,9637 \\
\hline 78 & 0,5794 & 1,1339 & 0,9669 & 0,5821 & 1,1317 & 0,9859 \\
\hline 79 & 0,5906 & 1,0549 & 0,9412 & 0,5930 & 1,0530 & 0,9460 \\
\hline 80 & 0,5973 & 1,0756 & 0,9574 & 0,6009 & 1,0733 & 0,9700 \\
\hline 81 & 0,6017 & 1,1173 & 0,9624 & 0,6057 & 1,1152 & 1,1514 \\
\hline 82 & 0,5541 & 1,1321 & 0,9702 & 0,5555 & 1,1295 & 0,8152 \\
\hline 83 & 0,5958 & 1,1585 & 0,9533 & 0,5989 & 1,1569 & 1,0146 \\
\hline 84 & 0,5645 & 1,1506 & 0,9634 & 0,5663 & 1,1487 & 0,9541 \\
\hline 85 & 0,5526 & 1,2050 & 0,9728 & 0,5540 & 1,2031 & 1,0657 \\
\hline 86 & 0,5683 & 1,1365 & 0,9744 & 0,5705 & 1,1339 & 0,9342 \\
\hline 87 & 0,5968 & 1,0594 & 0,9602 & 0,6005 & 1,0570 & 0,8936 \\
\hline
\end{tabular}


Tabela 5. Estimativas dos componentes de variância e do parâmetro de dispersão, obtidas pelo método da quase-verossimilhança (QV) e máxima verossimilhança (MV), para os experimentos simulados

\begin{tabular}{ccccccc}
\hline & \multicolumn{3}{c}{ QV } & \multicolumn{3}{c}{ MV } \\
\cline { 2 - 7 } Exp & $\hat{\sigma}_{t}^{2}$ & $\hat{\sigma}_{e}^{2}$ & $\hat{\phi}$ & $\hat{\sigma}_{i}^{2}$ & $\hat{\sigma}_{e}^{2}$ & $\hat{\phi}$ \\
\hline 88 & 0,5573 & 1,1411 & 0,9719 & 0,5590 & 1,1385 & 1,0976 \\
89 & 0,5844 & 1,1236 & 0,9667 & 0,5875 & 1,1215 & 1,0136 \\
90 & 0,5718 & 1,0953 & 0,9561 & 0,5739 & 1,0931 & 1,0469 \\
91 & 0,5600 & 1,0966 & 0,9724 & 0,5620 & 1,0939 & 1,0337 \\
92 & 0,5717 & 1,1072 & 0,9668 & 0,5740 & 1,1048 & 1,0724 \\
93 & 0,5461 & 1,1251 & 0,9709 & 0,5473 & 1,1225 & 0,8883 \\
94 & 0,5827 & 1,0032 & 0,9571 & 0,5858 & 1,0005 & 0,9698 \\
95 & 0,5668 & 1,1516 & 0,9639 & 0,5687 & 1,1498 & 0,9818 \\
96 & 0,6079 & 1,0552 & 0,9550 & 0,6122 & 1,0531 & 0,9551 \\
97 & 0,5677 & 1,0753 & 0,9704 & 0,5701 & 1,0726 & 0,9200 \\
98 & 0,5324 & 1,1008 & 0,9706 & 0,5493 & 1,0981 & 0,9232 \\
99 & 0,5560 & 1,1080 & 0,9665 & 0,5575 & 1,1055 & 0,9996 \\
100 & 0,5728 & 1,1271 & 0,9654 & 0,5751 & 1,1248 & 0,9182 \\
\hline
\end{tabular}

Verifica-se pelos resultados da Tabela 5, que as estimativas do parâmetro de dispersão $(\hat{\phi})$, para ambos os métodos de estimação, estão bem próximas a um, valor admitido e fixo para este no modelo binomial, indicando que a variabilidade está consistente com a distribuição assumida, isto é, os dados não fornecem evidências da presença de superdispersão ou subdispersão para o modelo assumido. 


\subsubsection{Coeficientes de herdabilidade}

As estimativas dos coeficientes de herdabilidade no sentido amplo, para os experimentos simulados, obtidas a partir dos componentes de variância, apresentados nas Tabelas 4 e 5, estão apresentados na Tabela 6.

Tabela 6. Estimativas dos coeficientes de herdabilidade no sentido amplo para os experimentos simulados

\begin{tabular}{cccccc}
\hline & \multicolumn{2}{c}{ Modelo linear misto clássico } & \multicolumn{2}{c}{ Modelo linear generalizado misto } \\
\cline { 2 - 6 } Exp & proporções & arco seno $\sqrt{y / n}$ & BOX-COX & QV & MV \\
\cline { 2 - 6 } 1 & $h^{2}$ & $h^{2}$ & $h^{2}$ & $h^{2}$ & $h^{2}$ \\
2 & 0,2751 & 0,2734 & 0,2795 & 0,3483 & 0,3499 \\
3 & 0,2771 & 0,2813 & 0,2799 & 0,3510 & 0,3528 \\
4 & 0,2247 & 0,2503 & 0,2364 & 0,3327 & 0,3259 \\
5 & 0,2755 & 0,2491 & 0,2740 & 0,3401 & 0,3339 \\
6 & 0,2514 & 0,2582 & 0,2548 & 0,3609 & 0,3416 \\
7 & 0,3435 & 0,3570 & 0,3520 & 0,3596 & 0,3625 \\
8 & 0,2331 & 0,2340 & 0,2462 & 0,3537 & 0,3553 \\
9 & 0,2368 & 0,2178 & 0,2379 & 0,3383 & 0,3398 \\
10 & 0,1253 & 0,1348 & 0,1278 & 0,3234 & 0,3242 \\
11 & 0,2774 & 0,2534 & 0,2792 & 0,3600 & 0,3619 \\
12 & 0,1955 & 0,1872 & 0,1977 & 0,3312 & 0,3325 \\
13 & 0,2074 & 0,2310 & 0,2283 & 0,3452 & 0,3467 \\
14 & 0,2455 & 0,2395 & 0,2452 & 0,3304 & 0,3315 \\
15 & 0,2132 & 0,2025 & 0,2133 & 0,3397 & 0,3411 \\
16 & 0,2672 & 0,2942 & 0,2616 & 0,3354 & 0,3344 \\
17 & 0,3127 & 0,2930 & 0,3248 & 0,3566 & 0,3584
\end{tabular}


Tabela 6. Estimativas dos coeficientes de herdabilidade no sentido amplo para os experimentos simulados

\begin{tabular}{|c|c|c|c|c|c|}
\hline & \multicolumn{3}{|c|}{ Modelo linear misto clássico } & \multicolumn{2}{|c|}{ Modelo linear generalizado misto } \\
\hline & proporções & $\operatorname{arcoseno} \sqrt{y / n}$ & $\mathrm{BOX}-\mathrm{COX}$ & QV & MV \\
\hline Exp & $h^{2}$ & $h^{2}$ & $h^{2}$ & $h^{2}$ & $h^{2}$ \\
\hline 18 & 0,1421 & 0,1316 & 0,1421 & 0,3457 & 0,3470 \\
\hline 19 & 0,1769 & 0,1956 & 0,1890 & 0,3195 & 0,3204 \\
\hline 20 & 0,2262 & 0,2441 & 0,2381 & 0,3506 & 0,3525 \\
\hline 21 & 0,2100 & 0,2281 & 0,2206 & 0,3337 & 0,3350 \\
\hline 22 & 0,2435 & 0,1940 & 0,2378 & 0,3365 & 0,3382 \\
\hline 23 & 0,1961 & 0,2102 & 0,2033 & 0,3173 & 0,3181 \\
\hline 24 & 0,2919 & 0,2867 & 0,2965 & 0,3409 & 0,3425 \\
\hline 25 & 0,2065 & 0,1856 & 0,2012 & 0,3214 & 0,3224 \\
\hline 26 & 0,2612 & 0,2545 & 0,2601 & 0,3369 & 0,3380 \\
\hline 27 & 0,2896 & 0,2902 & 0,2884 & 0,3536 & 0,3556 \\
\hline 28 & 0,2744 & 0,2652 & 0,2750 & 0,3517 & 0,3533 \\
\hline 29 & 0,2310 & 0,2567 & 0,2407 & 0,3357 & 0,3371 \\
\hline 30 & 0,1449 & 0,1251 & 0,1445 & 0,3244 & 0,3255 \\
\hline 31 & 0,2556 & 0,2683 & 0,2784 & 0,3466 & 0,3480 \\
\hline 32 & 0,2533 & 0,2123 & 0,2500 & 0,3468 & 0,3484 \\
\hline 33 & 0,2408 & 0,2087 & 0,2417 & 0,3401 & 0,3416 \\
\hline 34 & 0,2751 & 0,2396 & 0,2688 & 0,3376 & 0,3389 \\
\hline 35 & 0,3725 & 0,3649 & 0,3740 & 0,3576 & 0,3598 \\
\hline 36 & 0,3177 & 0,2763 & 0,3079 & 0,3527 & 0,3547 \\
\hline 37 & 0,2021 & 0,2166 & 0,2148 & 0,3218 & 0,3228 \\
\hline 38 & 0,2484 & 0,1991 & 0,2380 & 0,3412 & 0,3424 \\
\hline 39 & 0,3042 & 0,2952 & 0,3116 & 0,3365 & 0,3379 \\
\hline 40 & 0,2113 & 0,1778 & 0,2064 & 0,3329 & 0,3341 \\
\hline
\end{tabular}


Tabela 6. Estimativas dos coeficientes de herdabilidade no sentido amplo para os experimentos simulados

\begin{tabular}{|c|c|c|c|c|c|}
\hline & \multicolumn{3}{|c|}{ Modelo linear misto clássico } & \multicolumn{2}{|c|}{ Modelo linear generalizado misto } \\
\hline & proporções & $\operatorname{arcoseno} \sqrt{y / n}$ & BOX-COX & QV & MV \\
\hline Exp & $h^{2}$ & $h^{2}$ & $h^{2}$ & $h^{2}$ & $h^{2}$ \\
\hline 41 & 0,3310 & 0,3471 & 0,3354 & 0,3563 & 0,3584 \\
\hline 42 & 0,3592 & 0,3668 & 0,3665 & 0,3582 & 0,3602 \\
\hline 43 & 0,2824 & 0,2801 & 0,2868 & 0,3661 & 0,3679 \\
\hline 44 & 0,2016 & 0,2132 & 0,2099 & 0,3457 & 0,3470 \\
\hline 45 & 0,2759 & 0,2517 & 0,2751 & 0,3455 & 0,3471 \\
\hline 46 & 0,1955 & 0,2232 & 0,2011 & 0,3213 & 0,3222 \\
\hline 47 & 0,1884 & 0,1695 & 0,1852 & 0,3412 & 0,3426 \\
\hline 48 & 0,1832 & 0,2039 & 0,1954 & 0,3276 & 0,3288 \\
\hline 49 & 0,2368 & 0,1937 & 0,2165 & 0,3345 & 0,3358 \\
\hline 50 & 0,3122 & 0,2974 & 0,3089 & 0,3344 & 0,3358 \\
\hline 51 & 0,2401 & 0,2419 & 0,2440 & 0,3317 & 0,3329 \\
\hline 52 & 0,2354 & 0,2057 & 0,2287 & 0,3321 & 0,3334 \\
\hline 53 & 0,1579 & 0,1569 & 0,1597 & 0,3217 & 0,3224 \\
\hline 54 & 0,2027 & 0,1809 & 0,1996 & 0,3309 & 0,3319 \\
\hline 55 & 0,2137 & 0,2010 & 0,2163 & 0,3389 & 0,3402 \\
\hline 56 & 0,2733 & 0,2961 & 0,2823 & 0,3557 & 0,3570 \\
\hline 57 & 0,2796 & 0,2953 & 0,2840 & 0,3531 & 0,3547 \\
\hline 58 & 0,2010 & 0,2200 & 0,2066 & 0,3363 & 0,3374 \\
\hline 59 & 0,2416 & 0,2533 & 0,2483 & 0,3290 & 0,3302 \\
\hline 60 & 0,2488 & 0,2241 & 0,2488 & 0,3536 & 0,3553 \\
\hline 61 & 0,2037 & 0,2023 & 0,2007 & 0,3242 & 0,3252 \\
\hline 62 & 0,1302 & 0,1127 & 0,1279 & 0,3024 & 0,3030 \\
\hline 63 & 0,2711 & 0,2556 & 0,2771 & 0,3424 & 0,3439 \\
\hline
\end{tabular}


Tabela 6. Estimativas dos coeficientes de herdabilidade no sentido amplo para os experimentos simulados

\begin{tabular}{|c|c|c|c|c|c|}
\hline & \multicolumn{3}{|c|}{ Modelo linear misto clássico } & \multicolumn{2}{|c|}{ Modelo linear generalizado misto } \\
\hline & proporções & $\operatorname{arcoseno} \sqrt{y / n}$ & $\mathrm{BOX}-\mathrm{COX}$ & QV & MV \\
\hline Exp & $h^{2}$ & $h^{2}$ & $h^{2}$ & $h^{2}$ & $h^{2}$ \\
\hline 64 & 0,1786 & 0,1683 & 0,1753 & 0,3259 & 0,3269 \\
\hline 65 & 0,2164 & 0,2085 & 0,2193 & 0,3362 & 0,3373 \\
\hline 66 & 0,1994 & 0,2215 & 0,2102 & 0,3240 & 0,3249 \\
\hline 67 & 0,2317 & 0,2449 & 0,2387 & 0,3224 & 0,3235 \\
\hline 68 & 0,3000 & 0,2944 & 0,3009 & 0,3560 & 0,3581 \\
\hline 69 & 0,2474 & 0,2505 & 0,2517 & 0,3525 & 0,3541 \\
\hline 70 & 0,2092 & 0,2400 & 0,2224 & 0,3428 & 0,3442 \\
\hline 71 & 0,1508 & 0,1472 & 0,1449 & 0,3350 & 0,3362 \\
\hline 72 & 0,2016 & 0,1906 & 0,2112 & 0,3384 & 0,3397 \\
\hline 73 & 0,2849 & 0,2803 & 0,2898 & 0,3444 & 0,3459 \\
\hline 74 & 0,1831 & 0,1695 & 0,1765 & 0,3234 & 0,3243 \\
\hline 75 & 0,2661 & 0,2800 & 0,2765 & 0,3335 & 0,3347 \\
\hline 76 & 0,2552 & 0,2449 & 0,2530 & 0,3308 & 0,3320 \\
\hline 77 & 0,2598 & 0,2887 & 0,2661 & 0,3340 & 0,3352 \\
\hline 78 & 0,2847 & 0,2639 & 0,2850 & 0,3382 & 0,3396 \\
\hline 79 & 0,2528 & 0,3024 & 0,2721 & 0,3589 & 0,3603 \\
\hline 80 & 0,3002 & 0,3050 & 0,3135 & 0,3571 & 0,3589 \\
\hline 81 & 0,2622 & 0,2858 & 0,2726 & 0,3500 & 0,3520 \\
\hline 82 & 0,2508 & 0,2469 & 0,2542 & 0,3286 & 0,3297 \\
\hline 83 & 0,2776 & 0,3241 & 0,2974 & 0,3396 & 0,3411 \\
\hline 84 & 0,2250 & 0,2642 & 0,2401 & 0,3291 & 0,3302 \\
\hline 85 & 0,1772 & 0,1848 & 0,1936 & 0,3144 & 0,3153 \\
\hline 86 & 0,2839 & 0,2527 & 0,2726 & 0,3334 & 0,3347 \\
\hline
\end{tabular}


Tabela 6. Estimativas dos coeficientes de herdabilidade no sentido amplo para os experimentos simulados

\begin{tabular}{cccccc}
\hline & \multicolumn{3}{c}{ Modelo linear misto clássico } & Modelo linear generalizado misto \\
\cline { 2 - 6 } & proporções & arco seno $\sqrt{y / n}$ & BOX-COX & QV & MV \\
\cline { 2 - 6 } 87 & $h^{2}$ & $h^{2}$ & $h^{2}$ & $h^{2}$ & $h^{2}$ \\
88 & 0,3378 & 0,3228 & 0,3369 & 0,3604 & 0,3623 \\
89 & 0,2625 & 0,2564 & 0,2612 & 0,3421 & 0,3293 \\
90 & 0,2225 & 0,2336 & 0,2333 & 0,3430 & 0,3438 \\
91 & 0,2276 & 0,1972 & 0,2166 & 0,3381 & 0,33443 \\
92 & 0,2380 & 0,2229 & 0,2304 & 0,3405 & 0,3419 \\
93 & 0,2092 & 0,2066 & 0,2150 & 0,3268 & 0,3278 \\
94 & 0,2825 & 0,2616 & 0,2839 & 0,3674 & 0,3693 \\
95 & 0,2377 & 0,2504 & 0,2431 & 0,3298 & 0,3309 \\
96 & 0,3226 & 0,3159 & 0,3181 & 0,3655 & 0,3676 \\
97 & 0,2578 & 0,2609 & 0,2616 & 0,3455 & 0,3471 \\
98 & 0,2169 & 0,1893 & 0,2147 & 0,3260 & 0,3334 \\
99 & 0,2022 & 0,2025 & 0,2043 & 0,3341 & 0,3352 \\
100 & 0,2661 & 0,2523 & 0,2648 & 0,3370 & 0,3383 \\
\hline
\end{tabular}

\subsubsection{Comparação dos métodos}

Para comparação das estimativas dos coeficientes de herdabilidade no sentido amplo, para os experimentos simulados, apresentados na Tabela 6 , considerando os modelos ajustados sob o enfoque de modelos lineares mistos clássicos, nas diferentes escalas, e também sob o enfoque de modelos lineares generalizados mistos, através dos métodos de estimação: quase-verossimilhança (QV) e máxima verossimilhança (MV), 
aplicou-se o teste de Permutação de Fisher, cujos resultados estão apresentados na Tabela 7 .

Tabela 7. Comparação das estimativas dos coeficientes de herdabilidade no sentido amplo, para os experimentos simulados, considerando os diferentes modelos. MLMC - Modelo linear misto clássico. MLGM - Modelo linear generalizado misto

\begin{tabular}{lc}
\hline \multicolumn{1}{c}{ COMPARAÇÃO } & p-valor \\
\hline MLMC - proporções x MLMC - arco seno $\sqrt{y / n}$ & 0,6078 \\
MLMC - proporções x MLMC - BOX-COX & 0,5847 \\
MLMC - proporções x MLGM - QV & $<0,0001$ \\
MLMC - proporções x MLGM - MV & $<0,0001$ \\
MLMC - arco seno $\sqrt{y} / n \times$ MLMC - BOX-COX & 0,2957 \\
MLMC - arco seno $\sqrt{y} / n \times$ MLGM - QV & $<0,0001$ \\
MLMC - arco seno $\sqrt{y} / n \times$ MLGM - MV & $<0,0001$ \\
MLMC - BOX-COX x MLGM - QV & $<0,0001$ \\
MLMC - BOX-COX x MLGM - MV & $<0,0001$ \\
MLGM - QV x MLGM - MV & 0,8005 \\
\hline
\end{tabular}

Verifica-se pelos resultados apresentados na Tabela 7 (p-valor), que as estimativas dos coeficientes de herdabilidade, considerando-se um modelo linear misto clássico para os dados na escala original (proporções) e transformados, utilizando-se as transformações: arco seno $\sqrt{y / n}$ e BOX-COX, não diferem estatisticamente entre si.

Observa-se que existem diferenças significativas nas estimativas dos coeficientes de herdabilidade, considerando-se um modelo linear misto clássico para os dados na escala original (proporções) e transformados, utilizando-se as transformações: 
$\operatorname{arco} \operatorname{seno} \sqrt{y} / n$ e BOX-COX, em relação às estimativas dos coeficientes de herdabilidade, obtidas admitindo-se um modelo linear generalizado misto com função de ligação logística, através dos métodos de estimação: quase-verossimilhança (QV) e máxima verossimilhança (MV).

As estimativas dos coeficientes de herdabilidade, obtidas considerando-se um modelo linear generalizado misto com função de ligação logística, através dos métodos de estimação: quase-verossimilhança (QV) e máxima verossimilhança (MV) não apresentaram diferenças significativas entre si.

Resultados similares foram obtidos por Otsuk (1991), no qual considerando um modelo aleatório inteiramente ao acaso com dados balanceados, através de simulações, analisou o efeito da transformação sobre as estimativas dos componentes de variância, para os efeitos de tratamentos e resíduo, supondo distribuição Binomial e Poisson. $O$ autor verificou que, as proporções de variações explicadas não diferem muito na presença de transformações, confirmando as indicações da literatura de que para se estimarem os componentes de variância, não é necessário que os dados sigam uma distribuição normal.

\subsubsection{Seleção do modelo}

Na Tabela 8 são apresentadas as seguintes estimativas: $h^{2}$ médio, viés, erro padrão, mínimo, máximo, amplitude, para os coeficientes de herdabilidade no sentido amplo, apresentados na Tabela 6 , bem como os valores de referência dos coeficientes de herdabilidade no sentido amplo, obtidos a partir dos dados utilizados na simulação dos experimentos. 
Tabela 8. Estimativas médias do coeficiente de herdabilidade, viés, erro padrão, mínimo, máximo, amplitude, para os experimentos simulados, e valores de referência dos coeficientes de herdabilidade

\begin{tabular}{lccccc}
\hline & \multicolumn{3}{c}{ Modelo linear misto clássico } & \multicolumn{3}{c}{$\begin{array}{c}\text { Modelo linear generalizado } \\
\text { misto }\end{array}$} \\
\cline { 2 - 6 } & proporções & arco seno $\sqrt{y / n}$ & BOX-COX & QV & MV \\
$h^{2}$ médio & 0,2417 & 0,2388 & 0,2447 & 0,3393 & 0,3407 \\
Viés & 0,1420 & 0,1181 & 0,1407 & 0,0176 & 0,0181 \\
Erro padrão & 0,0049 & 0,0052 & 0,0050 & 0,0012 & 0,0013 \\
Mínimo & 0,1248 & 0,1127 & 0,1274 & 0,3024 & 0,3030 \\
Máximo & 0,3722 & 0,3668 & 0,3738 & 0,3674 & 0,3693 \\
Amplitude & 0,2474 & 0,2541 & 0,2464 & 0,0650 & 0,0663 \\
$h^{2}$ Referência & 0,0996 & 0,1207 & 0,1040 & 0,3217 & 0,3226 \\
\hline
\end{tabular}

Verifica-se pelas estatísticas apresentadas na Tabela 8, que os modelos ajustados sob o enfoque de modelos lineares generalizados mistos, para ambos os métodos de estimação (QV e MV), quando comparados aos modelos ajustados considerando a teoria de modelos lineares mistos clássicos, apresentaram em média, maiores estimativas para os coeficientes de herdabilidade, o que do ponto de vista genético é o ideal. Este resultado também pode ser observado pela análise da Figura 1, onde estão plotados os valores dos coeficientes de herdabilidade estimados para os experimentos simulados.

Pode-se verificar que as estimativas dos coeficientes de herdabilidade, obtidas considerando a abordagem de modelos lineares generalizados mistos, independente do método de estimação ( $Q V$ ou MV), têm melhores propriedades estatísticas, do que as obtidas sob o enfoque de modelos lineares mistos clássicos. Os coeficientes de herdabilidade apresentaram menor viés, e os valores médios das 100 estimativas, aproximaram consideravelmente do valor de referência. Os erros padrão 
estimados foram de menor magnitude e os coeficientes de herdabilidade estimados sob esse enfoque, apresentaram uma menor amplitude de variação.

$\mathrm{Na}$ Figura 2 estão plotados os valores para os coeficientes de herdabilidade estimados para os experimentos simulados, e os valores de referência dos coeficientes de herdabilidade, obtidos a partir dos dados utilizados na simulação dos experimentos, considerando o enfoque de modelos lineares mistos clássicos e modelos lineares generalizados mistos.

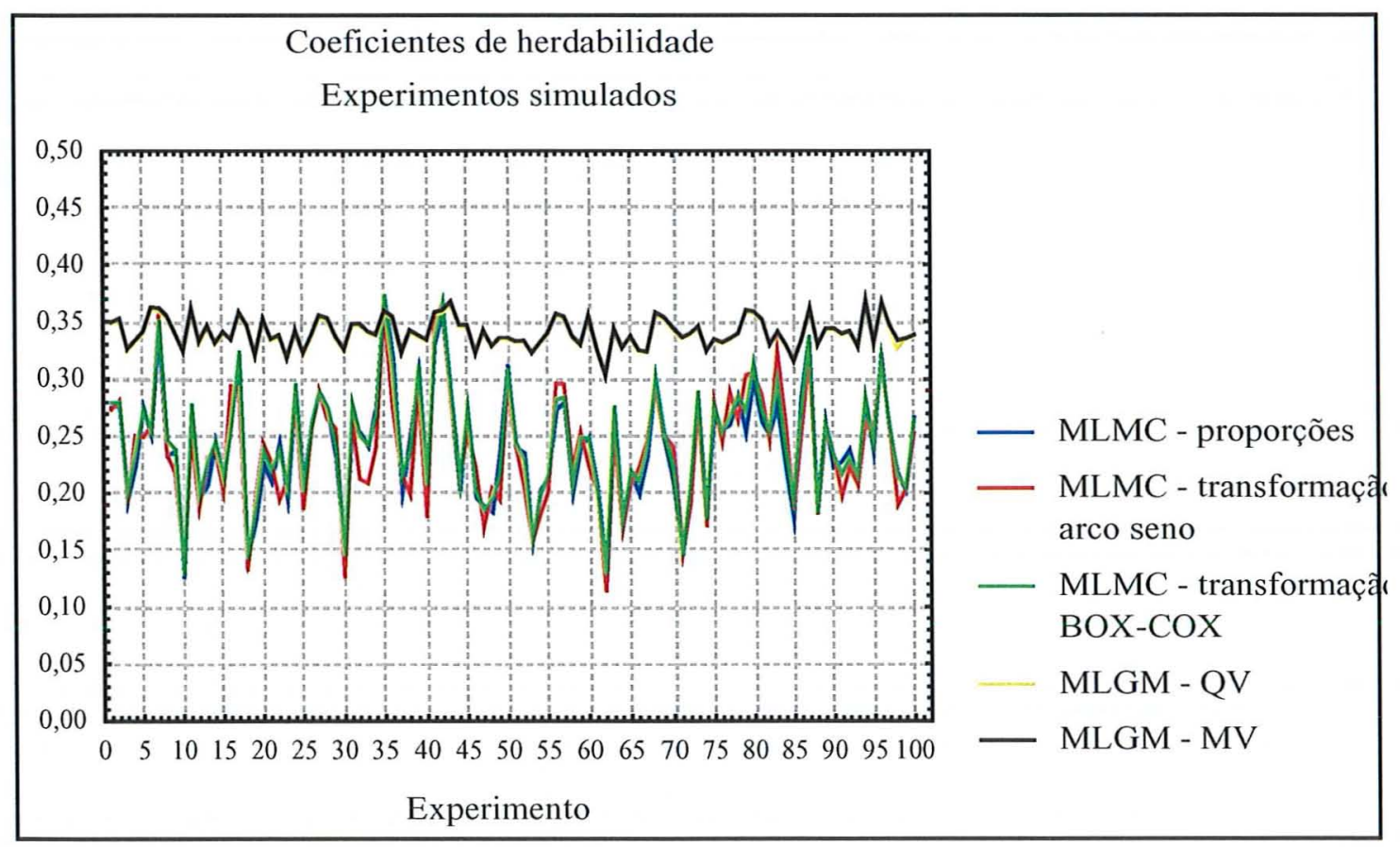

Figura 1 - Coeficientes de herdabilidade para os experimentos simulados. MLMC Modelo linear misto clássico. MLGM - Modelo linear generalizado misto 

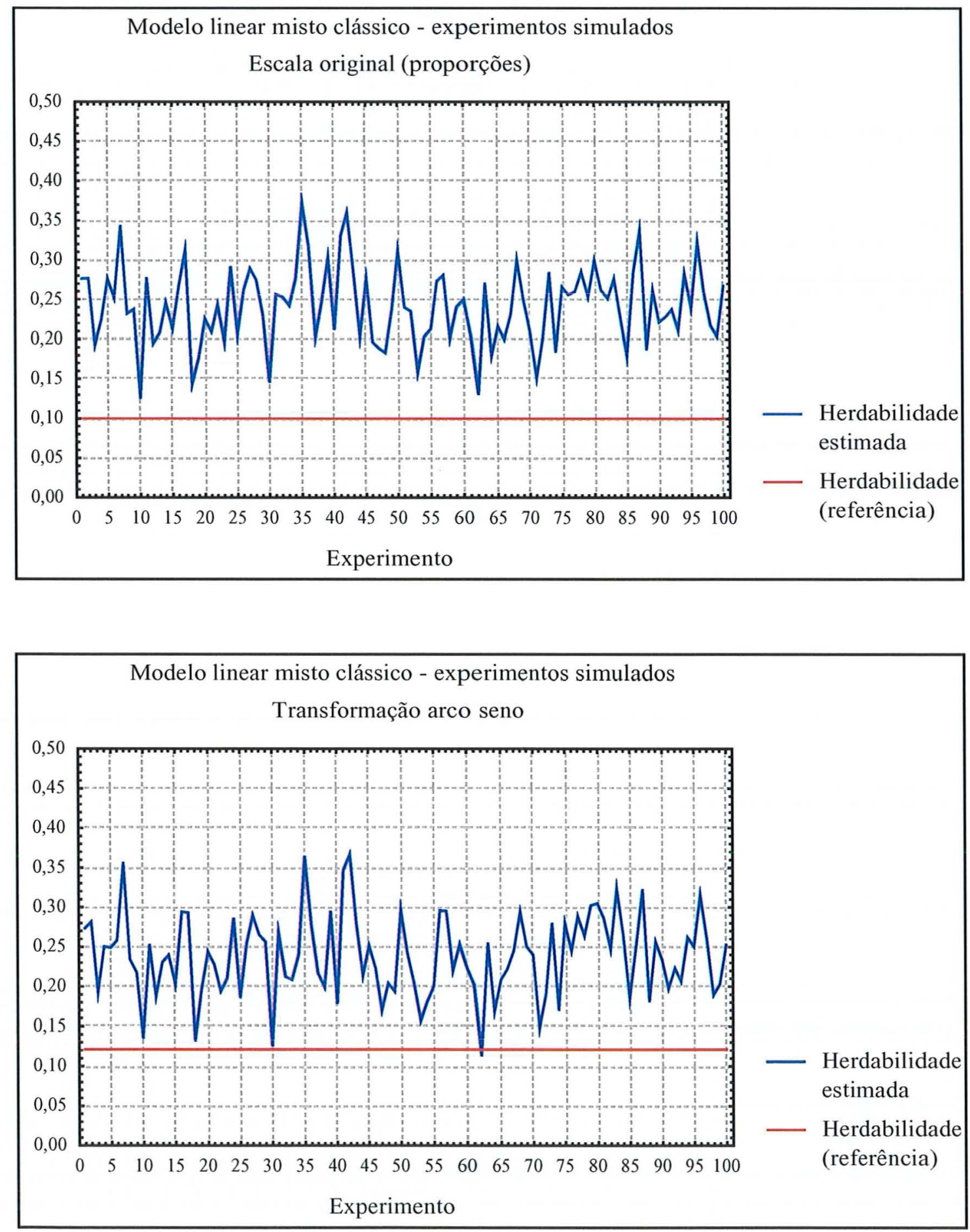

Figura 2 - Coeficientes de herdabilidade estimados para os experimentos simulados e valores de referência dos coeficientes de herdabilidade 

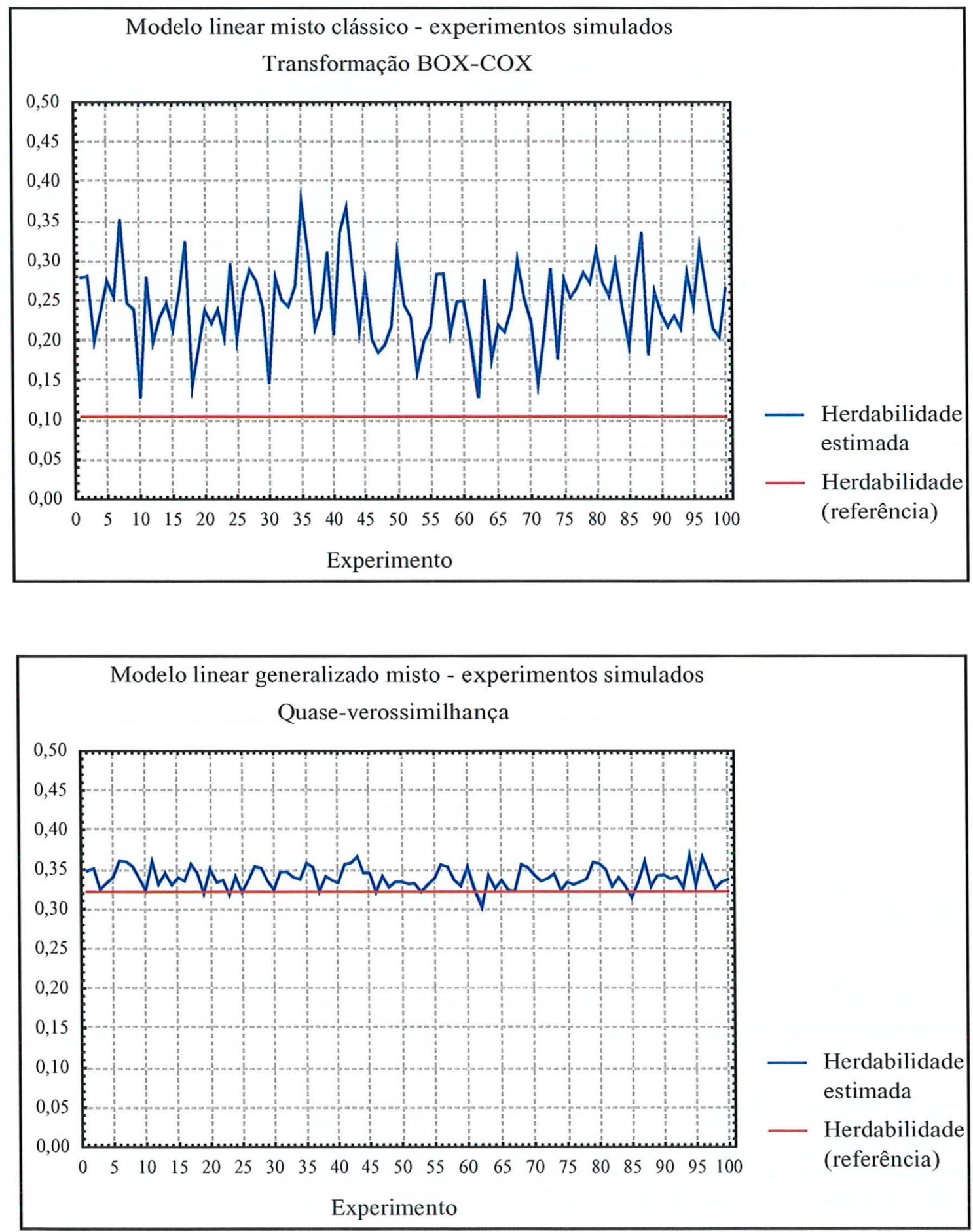

Figura 2 - Coeficientes de herdabilidade estimados para os experimentos simulados e valores de referência dos coeficientes de herdabilidade 


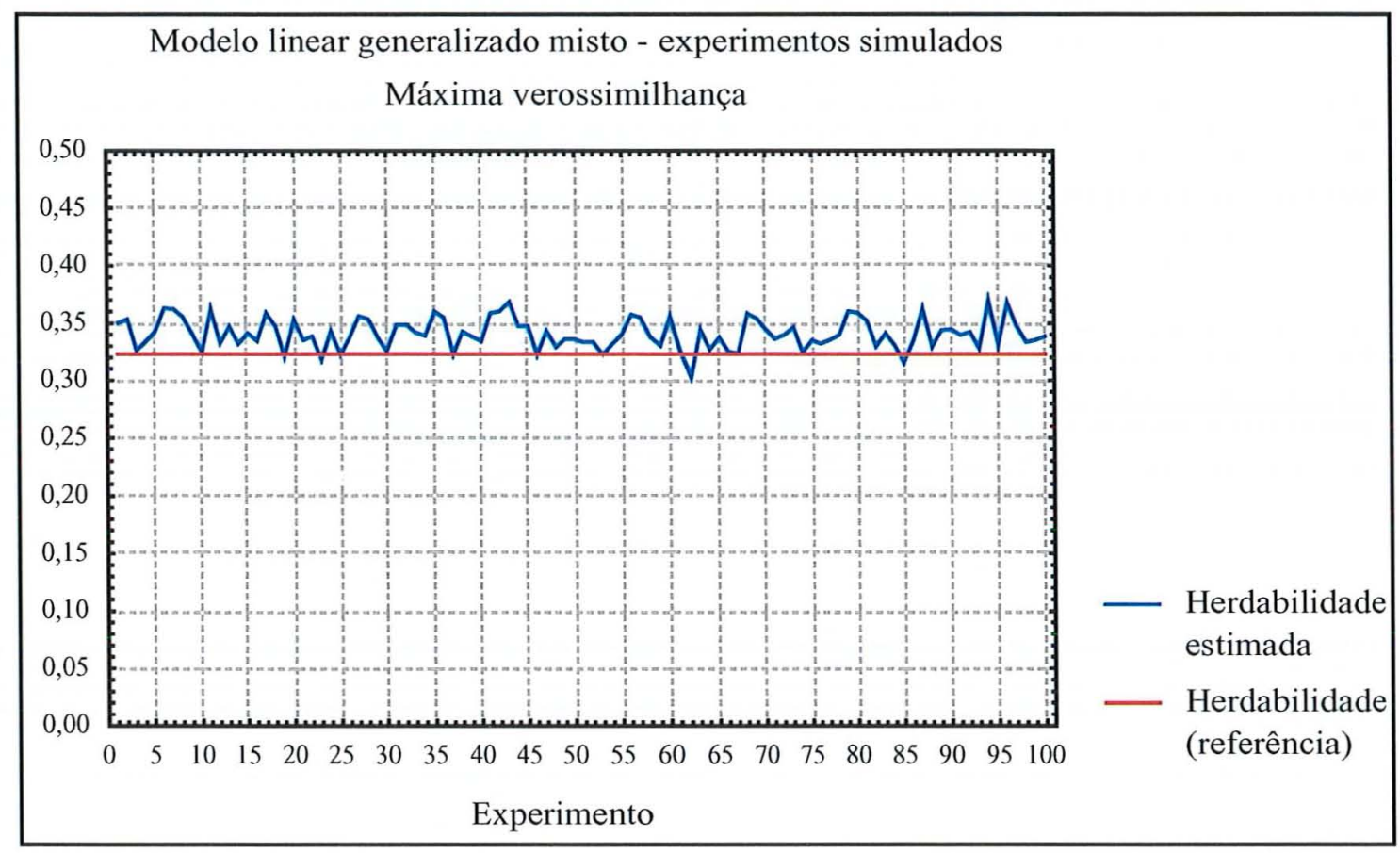

Figura 2 - Coeficientes de herdabilidade estimados para os experimentos simulados e valores de referência dos coeficientes de herdabilidade

Constata-se pelos resultados, para os experimentos simulados, que os modelos ajustados sob o enfoque de modelos lineares generalizados mistos com função de ligação logística, independente do método de estimação (QV ou MV), apresentaram um melhor ajuste, sendo, então, a metodologia preferida, quando se têm dados provenientes de proporções, para a estimação do coeficiente de herdabilidade.

\subsection{Dados de progênies de Eucalyptus grandis}

As estimativas dos componentes de variância para os efeitos genético de progênies $\left(\hat{\sigma}_{g}^{2}\right)$ e residual $\left(\hat{\sigma}_{e}^{2}\right)$, e seus erros padrão, com respectivas probabilidades, obtidas pelos métodos de estimação: quase-verossimilhança (QV) e máxima verossimilhança (MV), para os dados de plantas sobreviventes de Eucalyptus grandis, aos 5 anos de idade, num experimento em blocos ao acaso, considerando um modelo linear generalizado misto com função de ligação logística, são apresentados na Tabela 9. 
Tabela 9. Estimativas dos componentes de variância e seus erros padrão, com respectivas probabilidades, obtidas pelos métodos de estimação: quaseverossimilhança (QV) e máxima verossimilhança (MV), num modelo linear generalizado misto com função de ligação logística

QV MV

\begin{tabular}{ccccccc} 
Componentes & Estimativa & Erro padrão & $\mathrm{P}>\mathrm{t}$ & Estimativa & Erro padrão & $\mathrm{P}>\mathrm{t}$ \\
\hline$\sigma_{g}^{2}$ & 0,5803 & 0,0582 & 0,0000 & 0,5831 & 0,0609 & 0,0000 \\
$\sigma_{e}^{2}$ & 1,1819 & - & - & 1,1801 & - & -
\end{tabular}

$\mathrm{Na}$ Tabela 10 são apresentados as estimativas dos componentes de variância para os efeitos genético de progênies $\left(\hat{\sigma}_{g}^{2}\right)$ e residual $\left(\hat{\sigma}_{e}^{2}\right)$, e seus erros padrão, com respectivas probabilidades, obtidas pelo método de estimação da máxima verossimilhança, admitindo-se um modelo linear misto clássico, considerando-se os dados na escala original (proporções) e transformados, utilizando-se as transformações: $\operatorname{arcoseno} \sqrt{y / n}$ e BOX-COX.

Tabela 10. Estimativas dos componentes de variância e seus erros padrão, com respectivas probabilidades, obtidas pelo método de estimação da máxima verossimilhança, num modelo linear misto clássico em diferentes escalas

\begin{tabular}{ccccccc}
\hline & \multicolumn{3}{c}{$\sigma_{g}^{2}$} & & \multicolumn{3}{c}{$\sigma_{e}^{2}$} \\
Escalas & Estimativa & Erro padrão & $\mathrm{P}>\mathrm{Z}$ & Estimativa & Erro padrão & $\mathrm{P}>\mathrm{Z}$ \\
\hline proporções & 0,0126 & 0,0048 & 0,0044 & 0,0435 & 0,0041 & $<0,0001$ \\
arco seno & 76,4336 & 29,4649 & 0,0047 & 273,4100 & 25,7773 & $<0,0001$ \\
BOX-COX & 0,0149 & 0,0058 & 0,0049 & 0,0542 & 0,0051 & $<0,0001$ \\
\hline
\end{tabular}

As estimativas para o parâmetro de dispersão $(\hat{\phi})$, baseada na estatística de Pearson $X^{2}$ generalizada, considerando os métodos de estimação: quaseverossimilhança $(\mathrm{QV})$ e máxima verossimilhança $(\mathrm{MV})$, são respectivamente: 0,9772 e 
1,1867. Como esses valores estão bem próximos de um, valor admitido no modelo binomial, indicam que a variância está consistente com a distribuição assumida, isto é, os dados não fornecem evidências da presença de superdispersão ou subdispersão para o modelo assumido.

Os coeficientes de herdabilidade no sentido amplo, estimados considerando-se os componentes de variância apresentados na Tabela 9, foram: 0,3293 e 0,3307, respectivamente, para os métodos da quase-verossimilhança e máxima verossimilhança.

Considerando-se os componentes de variância apresentados na Tabela 10 , as estimativas para os coeficientes de herdabilidade no sentido amplo, para os dados na escala original (proporções) e transformados, utilizando-se as transformações: arco seno $\sqrt{y} / n$ e BOX-COX são: 0,2246, 0,2185 e 0,2156, respectivamente, valores estes bem inferiores aos estimados, considerando a teoria de modelos lineares generalizados mistos, conforme já evidenciados pelos experimentos simulados.

$\mathrm{Na}$ Tabela 11 estão apresentadas as estimativas dos efeitos fixos (blocos), pelo método da máxima verossimilhança considerando-se os modelos ajustados sob o enfoque de modelos lineares mistos clássicos, nas diferentes escalas, e também admitindo-se um modelo linear generalizado misto com função de ligação logística, pelos métodos de estimação: quase-verossimilhança $(\mathrm{QV})$ e máxima verossimilhança (MV). 
Tabela 11. Estimativas dos efeitos fixos (blocos), ajustados sob o enfoque de modelos lineares mistos clássicos, considerando as diferentes escalas, e admitindo-se um modelo linear generalizado misto com função de ligação logística, pelos métodos de estimação: quase-verossimilhança (QV) e máxima verossimilhança (MV)

\begin{tabular}{cccccc}
\hline & \multicolumn{3}{c}{ Modelo linear misto clássico } & \multicolumn{2}{c}{ Modelo linear generalizado misto } \\
\cline { 2 - 6 } Bloco & proporções & arco seno $\sqrt{y / n}$ & BOX-COX & QV & MV \\
1 & 0,6533 & 0,7088 & 0,6713 & 0,6602 & 0,6628 \\
2 & 0,6333 & 0,6611 & 0,6488 & 0,6392 & 0,6416 \\
3 & 0,7467 & 0,8102 & 0,7560 & 0,7570 & 0,7603 \\
4 & 0,7333 & 0,7823 & 0,7402 & 0,7433 & 0,7465 \\
5 & 0,6667 & 0,7021 & 0,6824 & 0,6741 & 0,6769 \\
6 & 0,7200 & 0,7852 & 0,7331 & 0,7296 & 0,7327 \\
7 & 0,7267 & 0,7675 & 0,7350 & 0,7365 & 0,7396 \\
8 & 0,7000 & 0,7531 & 0,7186 & 0,7089 & 0,7119 \\
9 & 0,6667 & 0,7112 & 0,6859 & 0,6741 & 0,6769 \\
10 & 0,7600 & 0,8075 & 0,7699 & 0,7707 & 0,7740 \\
\hline
\end{tabular}

Pode-se constatar pelas estimativas dos efeitos fixos (blocos), que estas apresentaram valores bem próximos, quando se ajustaram os modelos considerando o enfoque de modelos lineares mistos clássicos, através dos dados na escala original (proporções) e transformados através da transformação BOX-COX, com os modelos ajustados admitindo-se um modelo linear generalizado misto com função de ligação logística, pelos métodos de estimação: quase-verossimilhança (QV) e máxima verossimilhança (MV), indicando que esses modelos apresentaram ajustes bem próximos uns dos outros, o que também pode ser verificado pela Figura 3, onde estão plotados os valores para as estimativas de efeitos fixos (blocos), considerando os diferentes modelos de ajuste. Este fato também pode ser confirmado pelos valores das 
correlações de Spearman, apresentados na Tabela 12, onde se pode verificar que esses modelos apresentaram altas correlações, indicando que esses modelos apresentaram ajustes bastante coincidentes. Pode-se verificar também pelos valores das correlações de Spearman, que os modelos ajustados sob a abordagem de modelos lineares mistos clássicos, com os dados na escala original (proporções) e transformados através da transformação BOX-COX, coincidiram com os modelos ajustados sob o enfoque de modelos lineares generalizados mistos através dos métodos de estimação: quaseverossimilhança $(\mathrm{QV})$ e máxima verossimilhança (MV). Observa-se também que, a transformação arco seno $\sqrt{y / n}$ se diferenciou um pouco mais dos demais modelos, apresentando maiores estimativas para os efeitos.

Tabela 12. Valores da correlação de Spearman para as estimativas de efeitos fixos (blocos), considerando os diferentes modelos de ajuste. MLMC - Modelo linear misto clássico. MLGM - Modelo linear generalizado misto

\begin{tabular}{cccccc}
\hline Modelo & $\begin{array}{c}\text { MLMC } \\
\text { Proporções }\end{array}$ & $\begin{array}{c}\text { MLMC } \\
\text { arco seno }\end{array}$ & $\begin{array}{c}\text { MLMC } \\
\text { BOX-COX }\end{array}$ & $\begin{array}{c}\text { MLGM } \\
\text { QV }\end{array}$ & $\begin{array}{c}\text { MLGM } \\
\text { MV }\end{array}$ \\
\hline $\begin{array}{c}\text { MLMC } \\
\text { Proporções }\end{array}$ & - & 0,9301 & 0,9970 & 1,0000 & 1,0000 \\
MLMC & & - & 0,9394 & 0,9301 & 0,9301 \\
arco seno & & - & & & \\
MLMC & & & - & 0,9970 & 0,9970 \\
BOX-COX & & & - & 1,0000 \\
$\begin{array}{c}\text { MLGM } \\
\text { QV }\end{array}$ & & & & - \\
MLGM & & & & \\
MV & & & & & \\
\hline
\end{tabular}

Os efeitos aleatórios preditos, considerando os modelos ajustados sob o enfoque de modelos lineares mistos clássicos, considerando as diferentes escalas, e também admitindo-se um modelo linear generalizado misto com função de ligação logística, pelos métodos de estimação: quase-verossimilhança (QV) e máxima verossimilhança (MV), estão apresentados na Tabela 13. 


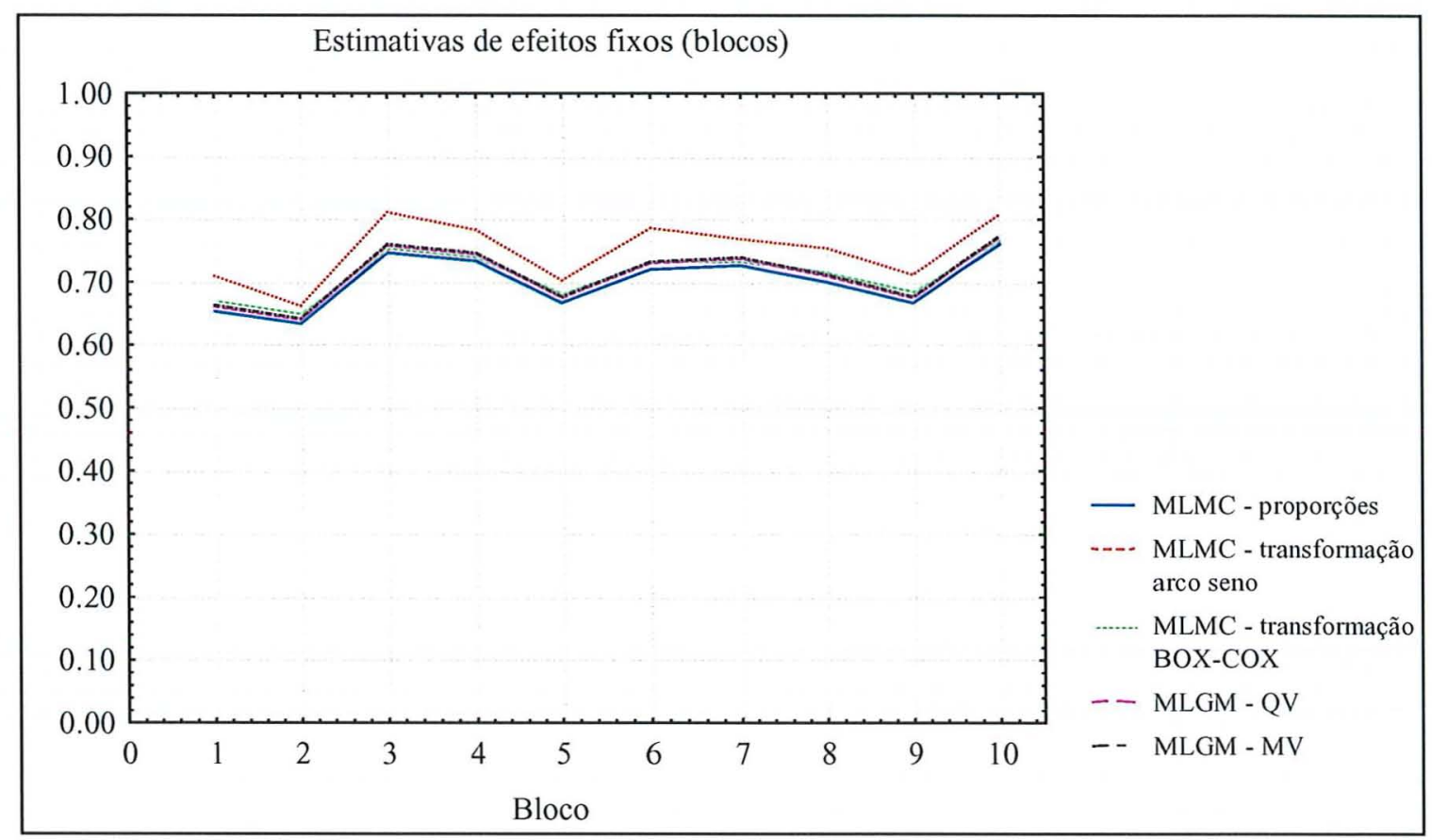

Figura 3 - Estimativas de efeitos fixos (blocos) para dados de plantas sobreviventes de Eucalyptus grandis, aos 5 anos de idade, num experimento em blocos ao acaso. MLMC - Modelo linear misto clássico. MLGM - Modelo linear generalizado misto

Tabela 13. Efeitos aleatórios preditos, ajustados sob o enfoque de modelos lineares mistos clássicos, considerando as diferentes escalas, e admitindo-se um modelo linear generalizado misto com função de ligação logística, pelos métodos de estimação: quase-verossimilhança (QV) e máxima verossimilhança (MV)

Modelo linear misto clássico

Modelo linear generalizado misto

$\begin{array}{llll}\text { Progênie proporções } \operatorname{arcoseno} \sqrt{y / n} & \text { BOX-COX } & \text { QV } & \text { MV }\end{array}$

$\begin{array}{llllll}1 & 0,6480 & 0,6892 & 0,6671 & 0,6532 & 0,6544 \\ 2 & 0,8834 & 0,9404 & 0,8851 & 0,8720 & 0,8731 \\ 3 & 0,8215 & 0,8705 & 0,8253 & 0,8204 & 0,8216\end{array}$


Tabela 13. Efeitos aleatórios preditos, ajustados sob o enfoque de modelos lineares mistos clássicos, considerando as diferentes escalas, e admitindo-se um modelo linear generalizado misto com função de ligação logística, pelos métodos de estimação: quase-verossimilhança (QV) e máxima verossimilhança (MV)

\begin{tabular}{|c|c|c|c|c|c|}
\hline \multirow[b]{2}{*}{ Progênie } & \multicolumn{3}{|c|}{ Modelo linear misto clássico } & \multicolumn{2}{|c|}{ Modelo linear generalizado misto } \\
\hline & proporções & $\operatorname{arcoseno} \sqrt{y / n}$ & $\mathrm{BOX}-\mathrm{COX}$ & QV & MV \\
\hline 4 & 0,7100 & 0,7661 & 0,7282 & 0,7163 & 0,7175 \\
\hline 5 & 0,8462 & 0,8880 & 0,8470 & 0,8416 & 0,8428 \\
\hline 6 & 0,7843 & 0,7991 & 0,7844 & 0,7871 & 0,7884 \\
\hline 7 & 0,6852 & 0,7414 & 0,7045 & 0,6915 & 0,6927 \\
\hline 8 & 0,7967 & 0,8133 & 0,7982 & 0,7984 & 0,7996 \\
\hline 9 & 0,8091 & 0,8619 & 0,8132 & 0,8095 & 0,8107 \\
\hline 10 & 0,7347 & 0,7560 & 0,7385 & 0,7405 & 0,7418 \\
\hline 11 & 0,8091 & 0,8490 & 0,8116 & 0,8095 & 0,8107 \\
\hline 12 & 0,7347 & 0,7575 & 0,7450 & 0,7405 & 0,7418 \\
\hline 13 & 0,7224 & 0,7579 & 0,7316 & 0,7285 & 0,7297 \\
\hline 14 & 0,9329 & 0,9693 & 0,9303 & 0,9093 & 0,9103 \\
\hline 15 & 0,8338 & 0,8665 & 0,8336 & 0,8311 & 0,8323 \\
\hline 16 & 0,8338 & 0,9029 & 0,8403 & 0,8311 & 0,8323 \\
\hline 17 & 0,7843 & 0,8151 & 0,7880 & 0,7871 & 0,7884 \\
\hline 18 & 0,8338 & 0,8800 & 0,8405 & 0,8311 & 0,8323 \\
\hline 19 & 0,7595 & 0,7953 & 0,7701 & 0,7641 & 0,7654 \\
\hline 20 & 0,6852 & 0,7118 & 0,6937 & 0,6915 & 0,6927 \\
\hline 21 & 0,4374 & 0,4361 & 0,4744 & 0,4161 & 0,4169 \\
\hline 22 & 0,6356 & 0,6602 & 0,6547 & 0,6402 & 0,6414 \\
\hline 23 & 0,7223 & 0,7611 & 0,7344 & 0,7285 & 0,7297 \\
\hline 24 & 0,7347 & 0,8019 & 0,7445 & 0,7405 & 0,7418 \\
\hline 25 & 0,8215 & 0,8578 & 0,8227 & 0,8204 & 0,8216 \\
\hline
\end{tabular}


Constata-se pelos resultados dos efeitos aleatórios preditos, apresentados na Tabela 13, que estes da mesma forma que para as estimativas dos efeitos fixos (blocos), apresentaram valores bem próximos, quando se ajustaram os modelos considerando o enfoque de modelos lineares mistos clássicos, através dos dados na escala original (proporções) e transformados através da transformação BOX-COX, com os modelos ajustados admitindo-se um modelo linear generalizado misto com função de ligação logística, pelos métodos de estimação: quase-verossimilhança (QV) e máxima verossimilhança (MV), indicando que esses modelos apresentaram ajustes bem próximos uns dos outros.

$\mathrm{Na}$ Figura 4, estão plotados os valores para os efeitos aleatórios preditos, considerando os diferentes modelos de ajuste, onde se pode observar que os modelos apresentaram ajustes bem próximos.

A Tabela 14 mostra os valores das correlações de Spearman, onde se observa que esses modelos apresentaram altas correlações, indicando que esses modelos apresentaram ajustes bastante coincidentes. Da mesma forma que para as estimativas dos efeitos fixos (blocos), verifica-se também pelos valores das correlações de Spearman, que os modelos ajustados sob a abordagem de modelos lineares mistos clássicos, com os dados na escala original (proporções) e transformados através da transformação BOXCOX, coincidiram com os modelos ajustados sob o enfoque de modelos lineares generalizados mistos através dos métodos de estimação: quase-verossimilhança (QV) e máxima verossimilhança (MV). Verifica-se também que, a transformação arco seno $\sqrt{y} / n$ se diferenciou um pouco mais dos demais modelos, apresentando maiores valores para os efeitos aleatórios preditos, apresentando uma maior capacidade de predição. 
Tabela 14. Valores da correlação de Spearman para os efeitos aleatórios preditos, considerando os diferentes modelos de ajuste. MLMC - Modelo linear misto clássico. MLGM - Modelo linear generalizado misto

\begin{tabular}{cccccc}
\hline Modelo & $\begin{array}{c}\text { MLMC } \\
\text { Proporções }\end{array}$ & $\begin{array}{c}\text { MLMC } \\
\text { arco seno }\end{array}$ & $\begin{array}{c}\text { MLMC } \\
\text { BOX-COX }\end{array}$ & $\begin{array}{c}\text { MLGM } \\
\text { QV }\end{array}$ & $\begin{array}{c}\text { MLGM } \\
\text { MV }\end{array}$ \\
\hline $\begin{array}{c}\text { MLMC } \\
\text { Proporções }\end{array}$ & - & 0,9703 & 0,9970 & 0,9998 & 0,9998 \\
MLMC & & - & 0,9746 & 0,9709 & 0,9709 \\
$\begin{array}{c}\text { arco seno } \\
\text { MLMC }\end{array}$ & & - & & 0,9975 & 0,9975 \\
BOX-COX & & & & - & 1,0000 \\
MLGM & & & & - \\
$\quad$ QV & & & & \\
MLGM & & & & & \\
MV & & & & & \\
\hline
\end{tabular}

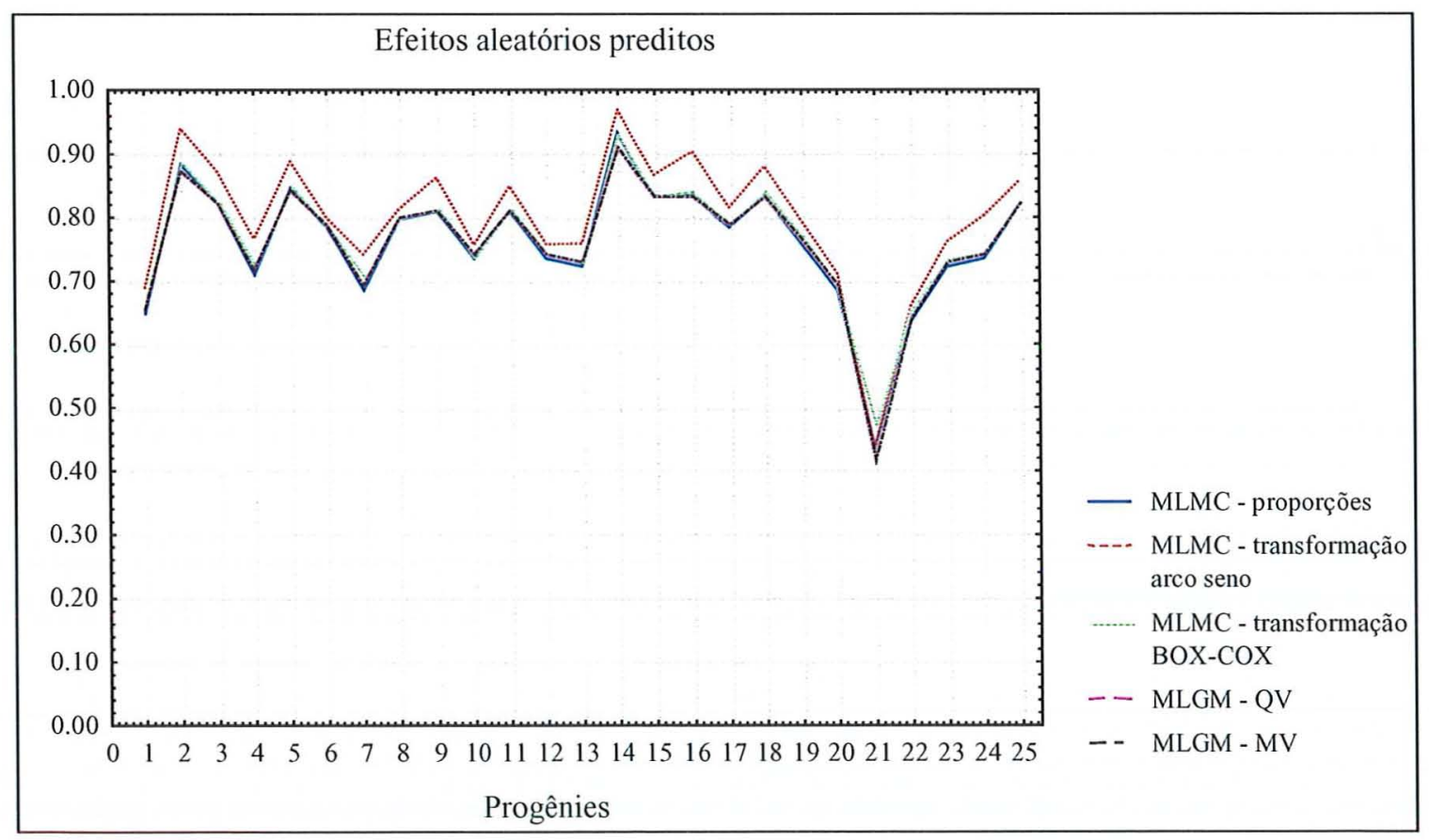

Figura 4 - Efeitos aleatórios preditos para dados de plantas sobreviventes de Eucalyptus grandis, aos 5 anos de idade, num experimento em blocos ao acaso. MLMC - Modelo linear misto clássico. MLGM - Modelo linear generalizado misto 
A Figura 5 mostra as proporções ajustadas de plantas sobreviventes de Eucalyptus grandis, aos 5 anos de idade, considerando os diferentes modelos de ajuste, onde também se pode observar, que com exceção do modelo ajustado sob o enfoque de modelos lineares mistos clássicos, através da transformação arco seno $\sqrt{y} / n$, que os demais modelos apresentaram ajustes bem próximos, o que também pode ser confirmado pelos valores das correlações de Spearman, apresentados na Tabela 15.

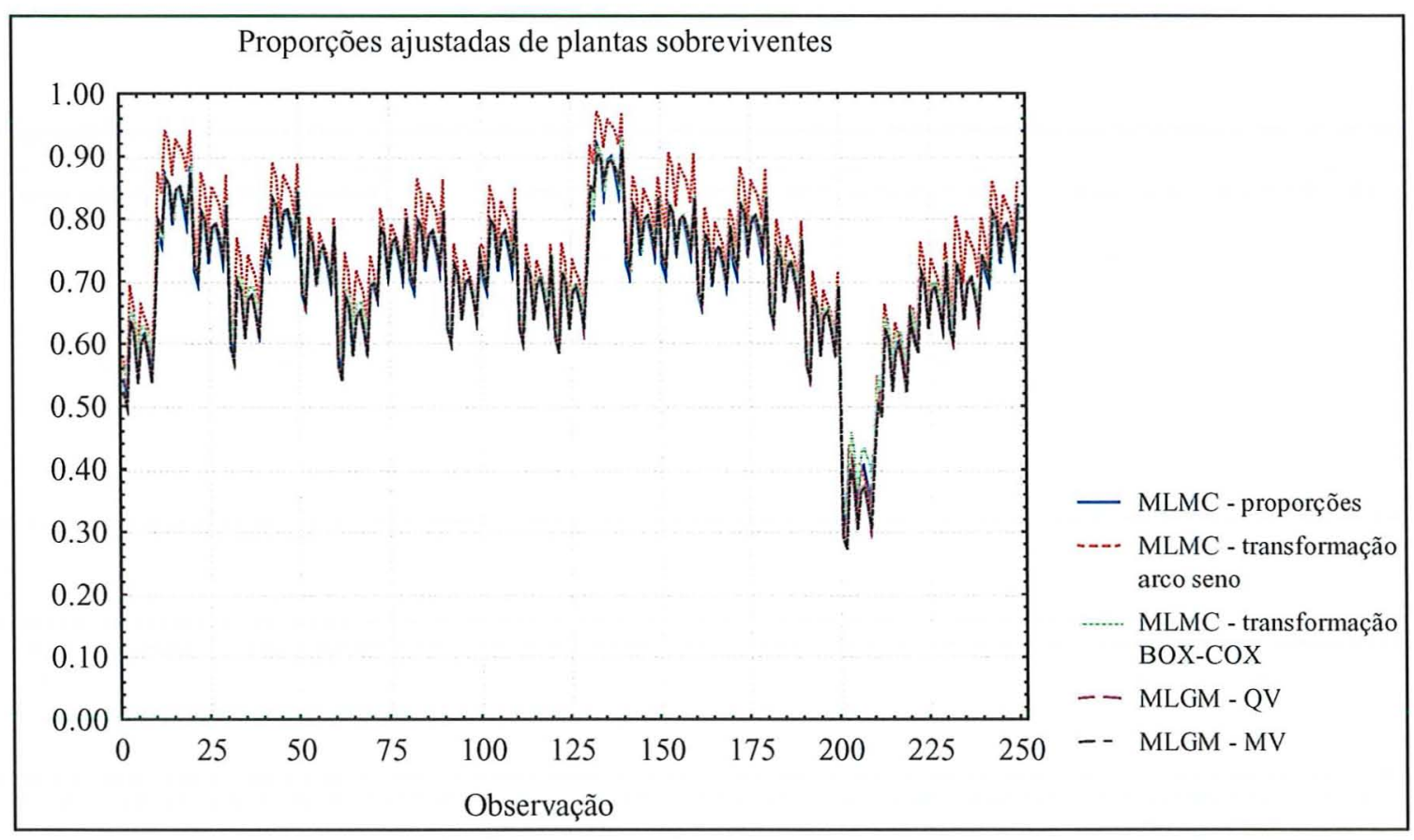

Figura 5 - Proporções ajustadas de plantas sobreviventes de Eucalyptus grandis, aos 5 anos de idade, num experimento em blocos ao acaso. MLMC - Modelo linear misto clássico. MLGM - Modelo linear generalizado misto 
Tabela 15. Valores da correlação de Spearman para as proporções ajustadas, considerando os diferentes modelos de ajuste. MLMC - Modelo linear misto clássico. MLGM - Modelo linear generalizado misto

\begin{tabular}{|c|c|c|c|c|c|}
\hline Modelo & $\begin{array}{c}\text { MLMC } \\
\text { Proporções }\end{array}$ & $\begin{array}{c}\text { MLMC } \\
\text { arco seno }\end{array}$ & $\begin{array}{c}\text { MLMC } \\
\text { BOX-COX }\end{array}$ & $\begin{array}{c}\text { MLGM } \\
\text { QV }\end{array}$ & $\begin{array}{c}\text { MLGM } \\
\text { MV }\end{array}$ \\
\hline $\begin{array}{c}\text { MLMC } \\
\text { Proporções }\end{array}$ & - & 0,9872 & 0,9988 & 0,9975 & 0,9975 \\
\hline $\begin{array}{l}\text { MLMC } \\
\text { arco seno }\end{array}$ & & - & 0,9911 & 0,9878 & 0,9878 \\
\hline $\begin{array}{c}\text { MLMC } \\
\text { BOX-COX }\end{array}$ & & & - & 0,9971 & 0,9971 \\
\hline $\begin{array}{c}\text { MLGM } \\
\text { QV }\end{array}$ & & & & - & 1,0000 \\
\hline $\begin{array}{c}\text { MLGM } \\
\text { MV }\end{array}$ & & & & & - \\
\hline
\end{tabular}

Além da análise do comportamento preditivo dos modelos, procedeu-se a uma análise visual dos resíduos para se verificar qual o modelo mais adequado. Assim, os gráficos para os resíduos, considerando os diferentes modelos de ajuste, estão apresentados na Figura 6. 

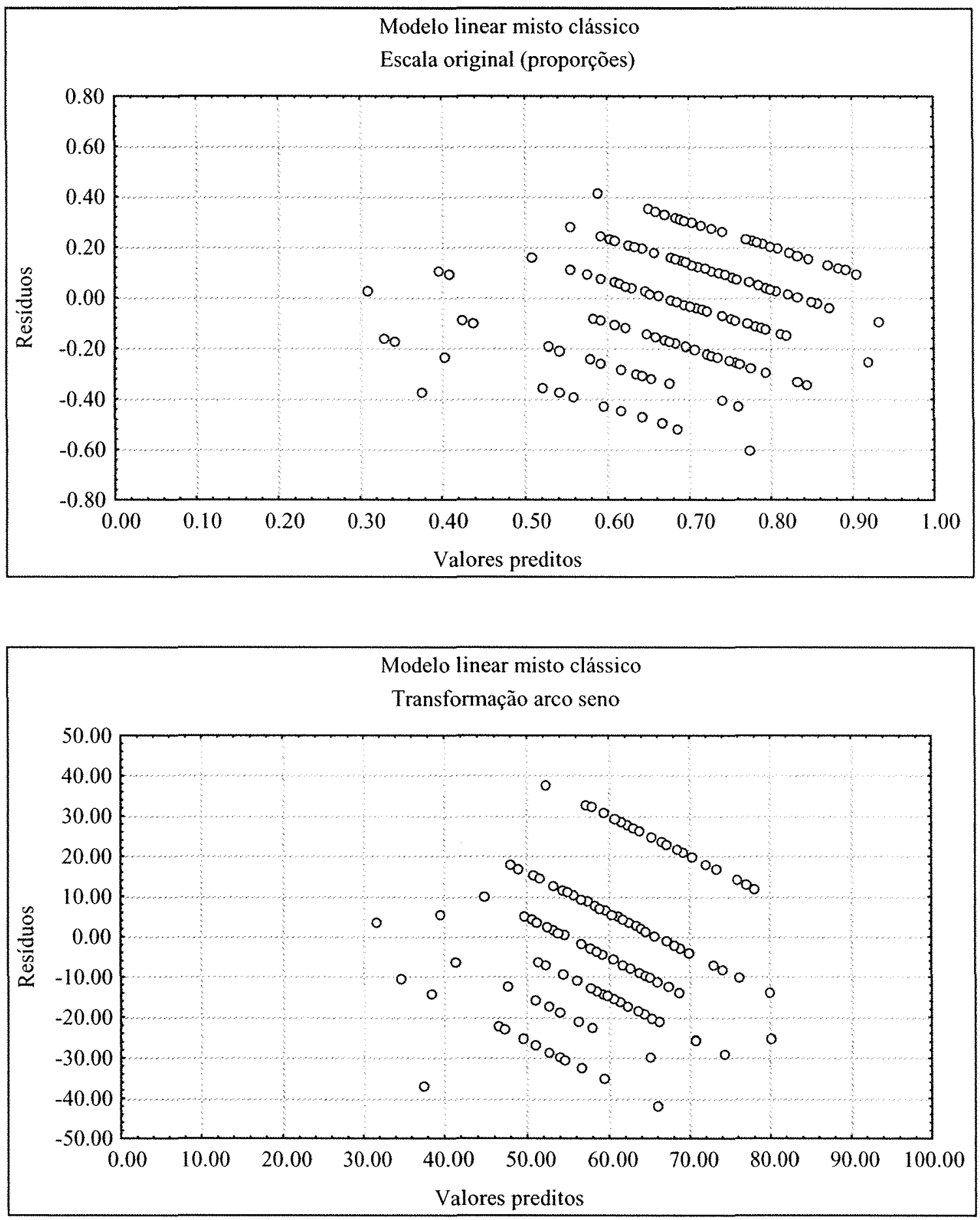

Figura 6 - Gráficos para os resíduos, considerando os diferentes modelos de ajuste, de plantas sobreviventes de Eucalyptus grandis, aos 5 anos de idade, num experimento em blocos ao acaso 

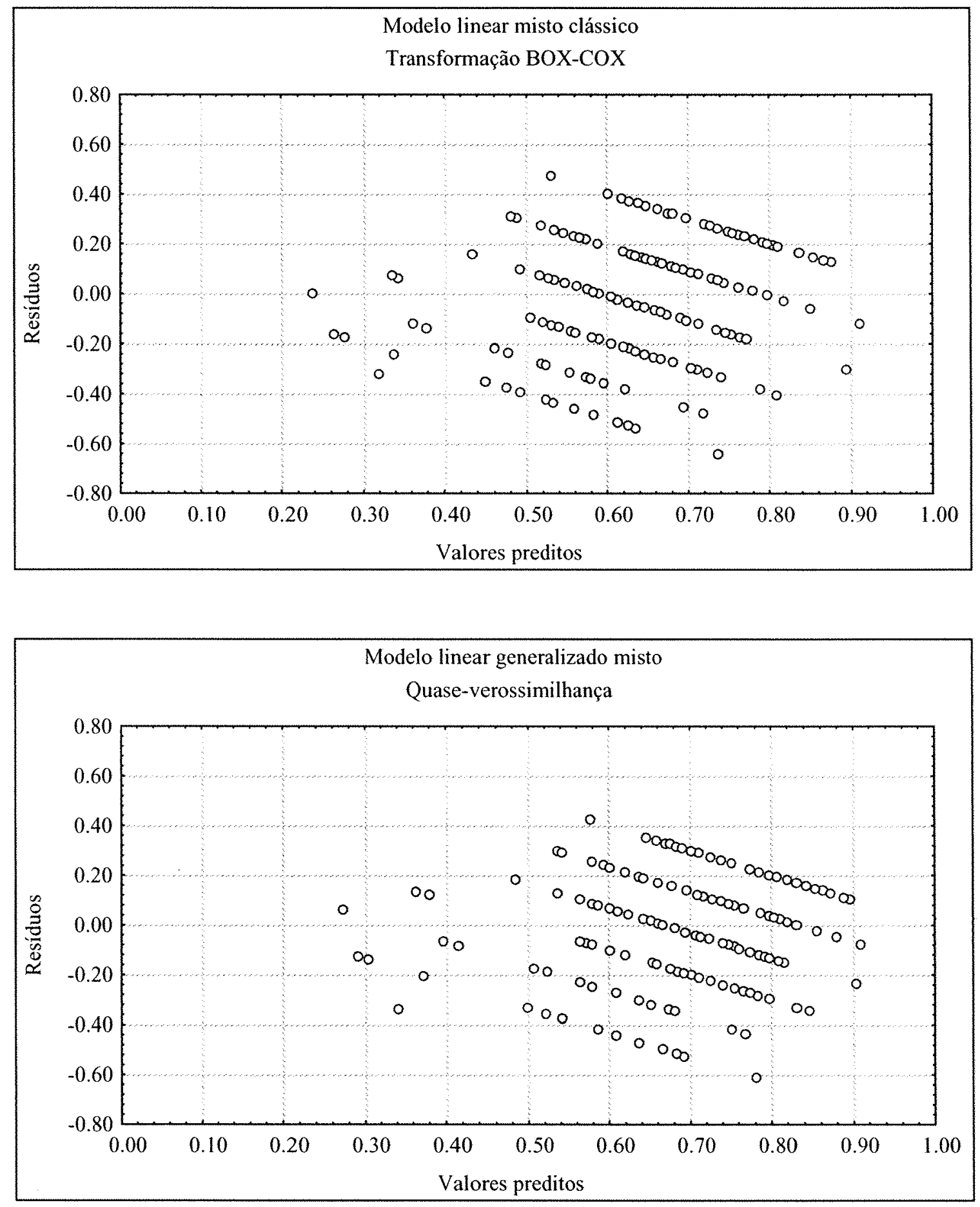

Figura 6 - Gráficos para os resíduos, considerando os diferentes modelos de ajuste, de plantas sobreviventes de Eucalyptus grandis, aos 5 anos de idade, num experimento em blocos ao acaso 


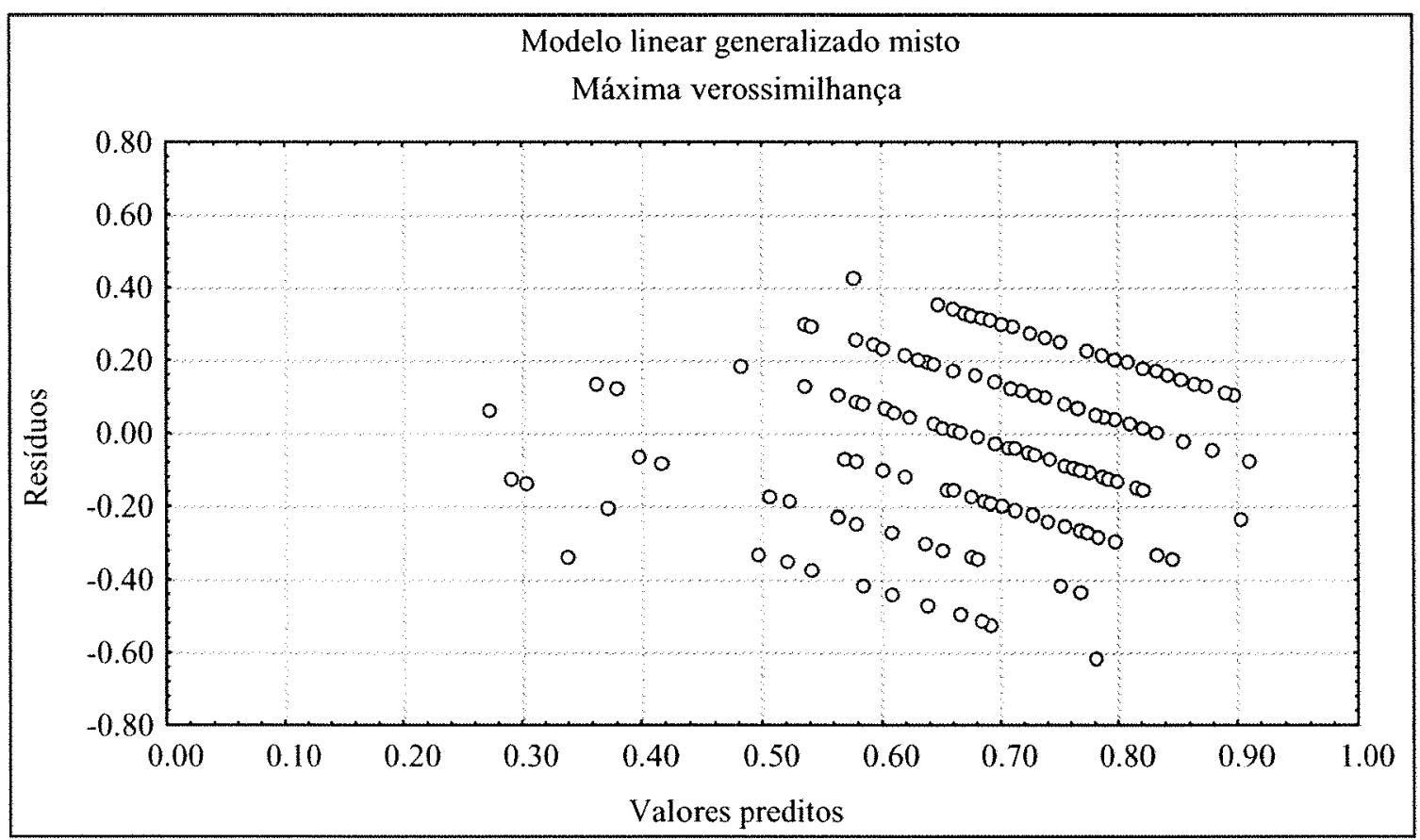

Figura 6 - Gráficos para os resíduos, considerando os diferentes modelos de ajuste, de plantas sobreviventes de Eucalyptus grandis, aos 5 anos de idade, num experimento em blocos ao acaso

Em uma visão geral dos gráficos da Figura 6, pode-se observar que com exceção do modelo ajustado sob o enfoque de modelos lineares mistos clássicos, através da transformação arco seno $\sqrt{y / n}$, que os demais modelos apresentaram ajustes bem parecidos, fato este já confirmado pelos resultados anteriores.

A Figura 7 mostra os gráficos $Q-Q$ Plot para os diferentes modelos ajustados, onde também se pode confirmar os resultados já obtidos. 
Modelo linear misto clássico

Escala original (proporções)

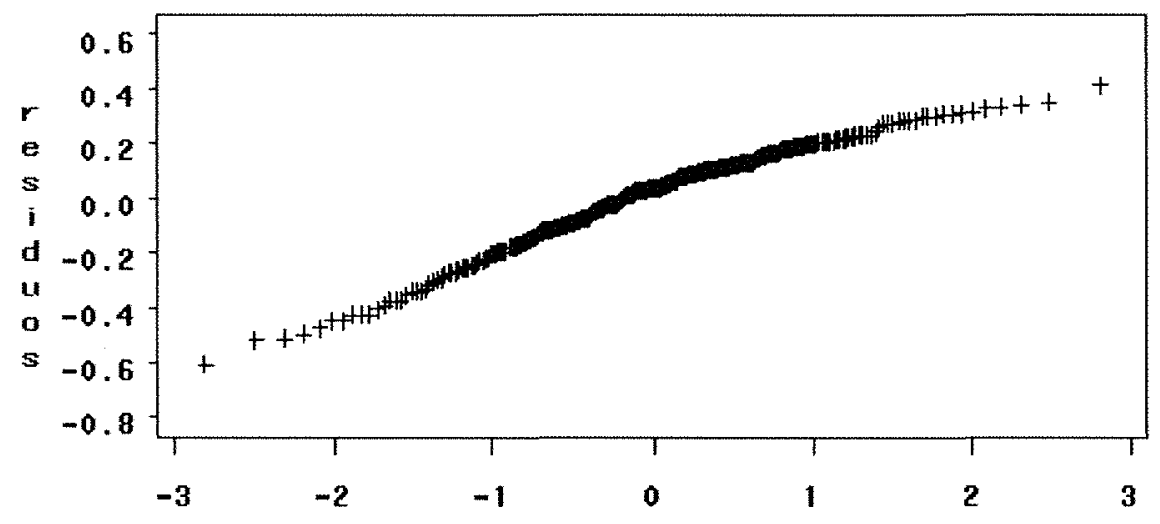

Quantis normais

Modelo linear misto clássico

Transformação arco seno

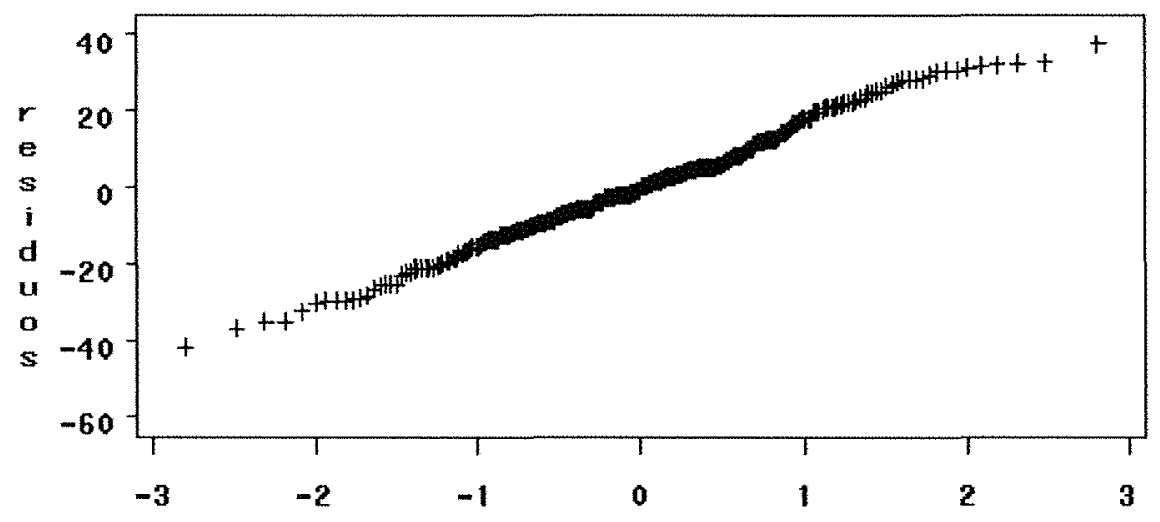

Quantis normais

Figura 7 - Gráficos $Q-Q$ Plot, considerando os diferentes modelos de ajuste, de plantas sobreviventes de Eucalyptus grandis, aos 5 anos de idade, num experimento em blocos ao acaso 

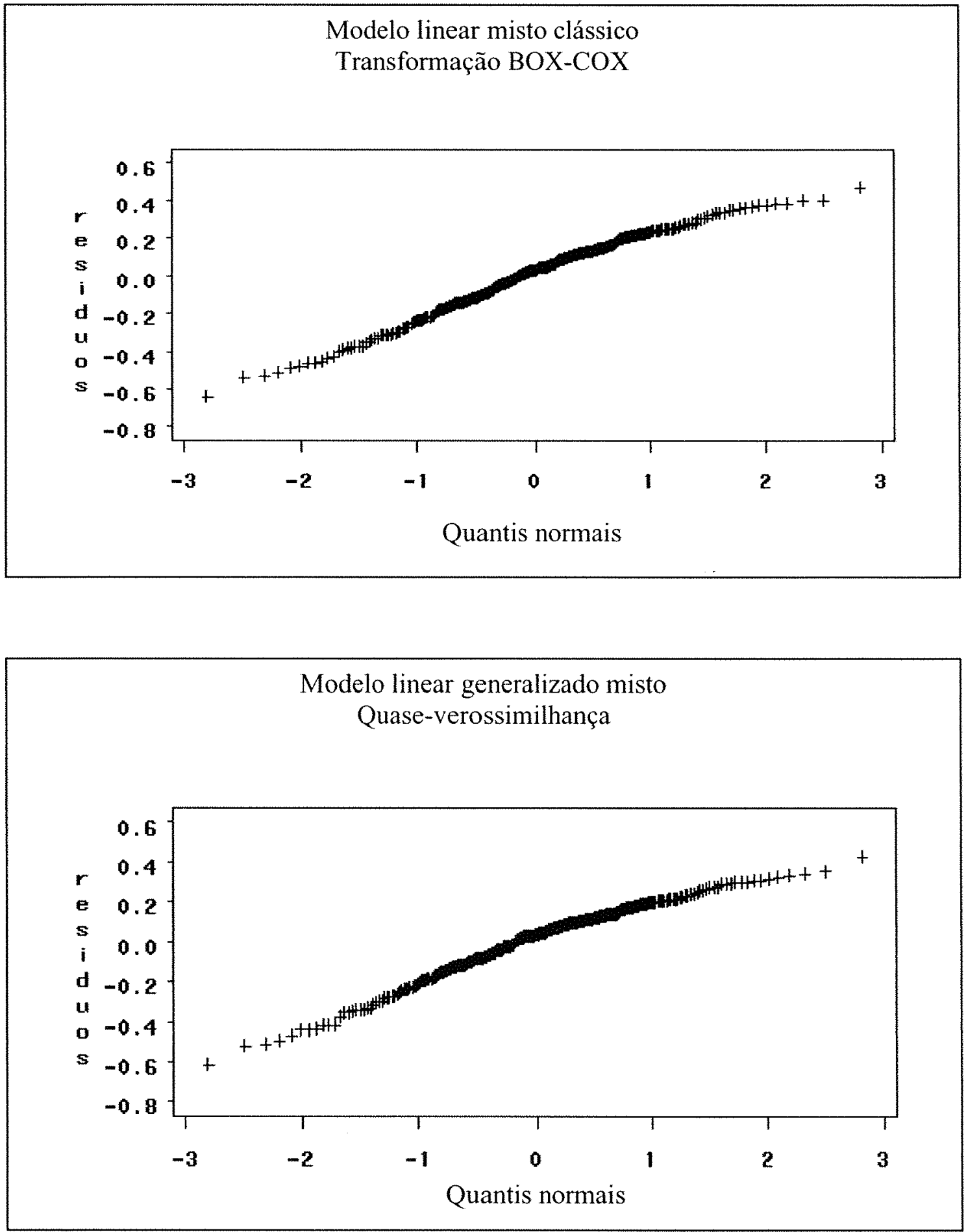

Figura 7 - Gráficos Q-Q Plot, considerando os diferentes modelos de ajuste, de plantas sobreviventes de Eucalyptus grandis, aos 5 anos de idade, num experimento em blocos ao acaso 
Modelo linear generalizado misto

Máxima verossimilhança

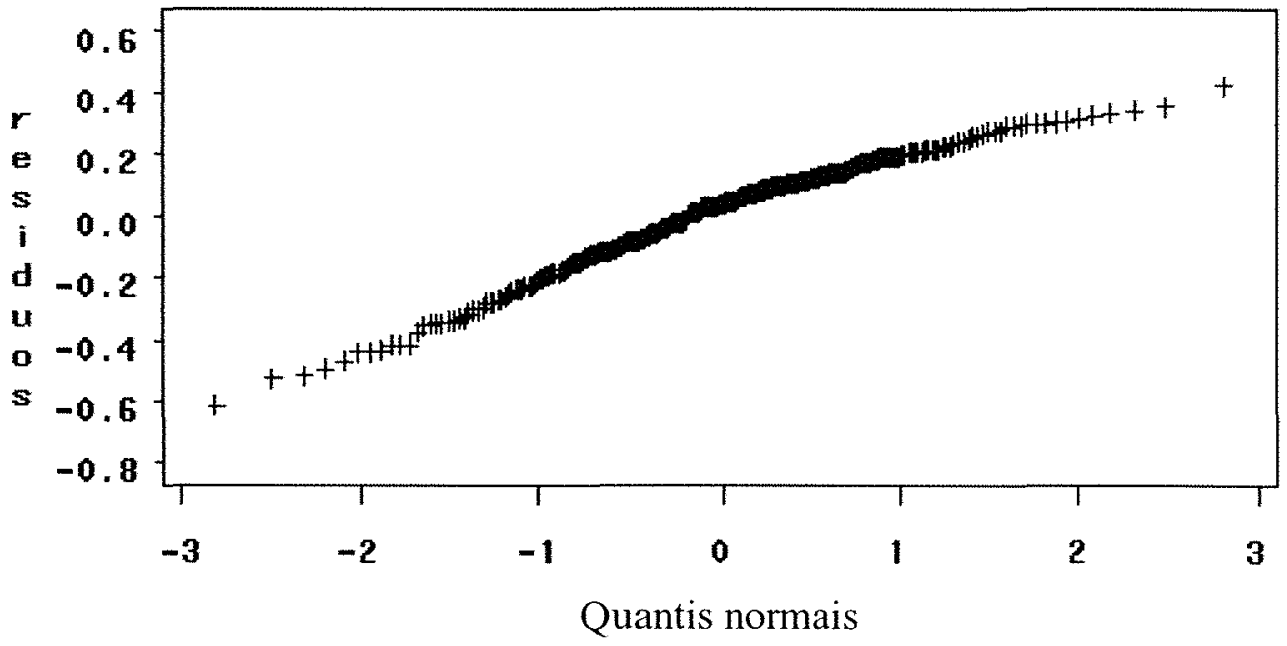

Figura 7 - Gráficos Q-Q Plot, considerando os diferentes modelos de ajuste, de plantas sobreviventes de Eucalyptus grandis, aos 5 anos de idade, num experimento em blocos ao acaso

De uma forma geral, verifica-se que o ajuste do modelo, através da teoria de modelos lineares mistos clássicos, com os dados transformados, por meio da transformação $\mathrm{BOX}-\mathrm{COX}$, não alterou a qualidade do ajuste em relação ao modelo ajustado com os dados na escala original (proporções). Pôde-se constatar também que esses modelos não se diferenciaram, em relação aos modelos ajustados, considerando o enfoque de modelos lineares generalizados mistos, para ambos os métodos de estimação (QV e MV). Por outro lado, verificou-se que o modelo ajustado por meio da transformação arco seno $\sqrt{y / n}$, considerando a abordagem de modelos lineares mistos clássicos, se diferenciou dos demais modelos, apresentando uma maior capacidade de predição, confirmando os referenciais da literatura, sobre a qualidade e importância do uso dessa transformação, quando se têm dados provenientes de proporções. 


\section{CONCLUSÕES}

A partir dos resultados obtidos, com base nos experimentos simulados, pode-se concluir que:

1. De uma maneira geral os modelos usados apresentaram-se adequados aos objetivos propostos;

2. Quando se têm dados de proporções, as estimativas dos coeficientes de herdabilidade no sentido amplo, não diferem na presença de transformações;

3. As estimativas dos coeficientes de herdabilidade obtidas com os dados transformados, através da transformação BOX-COX não mostraram diferenças em relação às obtidas com o uso da transformação arco seno $\sqrt{y / n}$. Assim, não é necessário o uso da transformação BOX-COX no caso de dados de proporções;

4. As estimativas dos coeficientes de herdabilidade para os dados originais e transformados, obtidas através da teoria de modelos lineares mistos clássicos, diferiram das estimativas obtidas considerando-se um modelo linear generalizado misto com função de ligação logística, através dos métodos de estimação da quaseverossimilhança e máxima verossimilhança;

5. Os métodos de estimação da quase-verossimilhança e máxima verossimilhança para o modelo linear generalizado misto com função de ligação logística, não diferiram quanto às estimativas dos coeficientes de herdabilidade;

6. Quando se têm dados de proporções, recomenda-se o uso de modelos lineares generalizados mistos, e dos métodos de estimação apresentados, para a estimação do coeficiente de herdabilidade. 
Em relação aos dados de proporções de plantas sobreviventes de Eucalyptus grandis, aos 5 anos de idade, num experimento em blocos ao acaso, foi possível concluir que:

1. O ajuste do modelo, através da teoria de modelos lineares mistos clássicos, com os dados transformados, por meio da transformação BOX-COX, não alterou a qualidade do ajuste em relação ao modelo ajustado com os dados na escala original (proporções), produzindo as mesmas estimativas para os efeitos fixos e predição para os efeitos aleatórios;

2. Os modelos ajustados sob o enfoque de modelos lineares mistos clássicos, com os dados na escala original (proporções), e transformados através da transformação BOX-COX, não se diferenciaram em relação aos modelos ajustados, considerando o enfoque de modelos lineares generalizados mistos com função de ligação logística, para ambos os métodos de estimação: quase-verossimilhança e máxima verossimilhança, produzindo estimativas para os efeitos fixos e predição para os efeitos aleatórios bastante semelhantes;

3. O método de estimação da quase-verossimilhança, para o modelo linear generalizado misto com função de ligação logística, apresentou as mesmas estimativas dos efeitos fixos e predição para os efeitos aleatórios, em relação aos obtidos pelo método da máxima verossimilhança;

4. O modelo ajustado por meio da transformação arco seno $\sqrt{y} / n$, considerando a abordagem de modelos lineares mistos clássicos, se diferenciou dos demais modelos, apresentando uma maior capacidade de predição. 


\section{ANEXOS}


ANEXO A - Programa SAS para a verificar pressuposição de normalidade para os experimentos simulados.

options nodate nonumber;

data simula;

infile 'C: Meus documentos\TESE\Arquivos Sas\Dados.prn';

input exp tra bl y;

$\mathrm{p}=\mathrm{y} / 6$;

$\mathrm{t}=\operatorname{arsin}(\mathrm{sqrt}(\mathrm{p}))^{*} 180 / 3.1415927$;

box $=\mathrm{p}^{* *} 1.5$;

run;

proc sort;

by exp;

run;

proc univariate normal plot;

var p t box;

by exp;

run; 
ANEXO B - Programa SAS para análise dos experimentos simulados, considerando o modelo linear misto clássico.

options nodate nonumber;

data simula;

infile 'C: Meus documentos\TESE\Arquivos Sas\Dados.prn';

input exp tra bl y;

$\mathrm{p}=\mathrm{y} / 6$;

$\mathrm{t}=\operatorname{arsin}(\operatorname{sqrt}(\mathrm{p}))^{*} 180 / 3.1415927$;

box $=\mathrm{p}^{* *} 1.5$;

run;

proc sort;

by exp;

run;

proc mixed method=ML covtest ic;

class tra bl;

model $\mathrm{p} t \mathrm{box}=\mathrm{bl}$;

random tra;

by exp;

run; 
ANEXO C - Programa SAS para análise dos experimentos simulados, considerando o modelo linear generalizado misto com função de ligação logística, pelo método da quase-verossimilhança.

options nodate nonumber;

data simula;

infile 'C: Meus documentos\TESE\Arquivos Sas \Glimmix.prn';

input exp bloco trat $\mathrm{s}$ ns;

run;

proc sort;

by exp;

run;

data new;

set simula;

do $\mathrm{i}=1$ to $\mathrm{s}$;

$y=1$;

output;

end;

do $\mathrm{i}=1$ to ns;

$y=0$;

output;

end;

\%include

'C: \Arquivos

de

programas $\backslash$ SAS

InstitutelSAS $\backslash V 8 \backslash S T A T \backslash S A M P L E \backslash g l i m m i x . s a s ' ;$

$\%$ glimmix $($ data $=$ new, procopt $=$ method $=m l$ ic,

stmts $=\%$ str (class trat bloco;

model $y=$ bloco;

by exp;

random trat;

), 
error-binomial, link=logit, out=pred);

run;

proc print data $=$ pred $(\mathrm{keep}=\exp \mathrm{mu})$ noobs; run; 
ANEXO D - Programa SAS para análise dos experimentos simulados, considerando o modelo linear generalizado misto com função de ligação logística, pelo método da máxima verossimilhança.

options nodate nonumber;

data simula;

input y $\mathrm{n}$ Bloco Trat;

cards;

*dados* $/ *$ Entra com os dados*/

;

proc glmmod data $=$ simula outdesign $=$ simul;

class Bloco Trat;

model y $n$ Trat=Bloco/noint;

run;

proc sort data $=$ simul;

by Trat;

run;

proc nlmixed data $=$ simul qpoints $=16$;

parms Bloco1 Bloco2 Bloco3 Bloco4 Bloco5 Bloco6 Bloco7 Bloco8 Bloco9 Bloco10=0

$\operatorname{sig} 2 \mathrm{p}=1$

array $x\{10\}$ coll-collo;

array beta $\{10\}$ Bloco1--Bloco10;

eta $=$;

do $\mathrm{i}=1$ to 10 ;

eta $=$ eta $+x\{i\} *$ beta $\{i\}$;

end;

$\mathrm{p}=\exp ($ eta $) /(1+\exp ($ eta $))$;

model y $\sim \operatorname{binomial}(n, p)$;

random $u \sim \operatorname{normal}(0$, sig $2 \mathrm{p})$ subject $=$ Trat;

predict $\mathrm{p}$ out $=\mathrm{p}$; 
run;

proc print data $=p($ keep $=$ pred $)$ noobs;

run; 
ANEXO E - Programa SAS para comparação dos coeficientes de herdabilidade para os experimentos simulados.

options nodate nonumber;

data simula;

input tra h;

cards;

*dados* /*Entra com os valores dos coeficientes de herdabilidade*/

;

proc print;

run;

proc multtest data $=$ simula permutation nsample $=10000 \mathrm{seed}=28281$;

class tra;

test mean(h);

contrast; /*Entra com o contraste de interesse*/

run; 
ANEXO F - Programa SAS para análise dos dados de progênies de Eucalyptus grandis, considerando o modelo linear misto clássico.

options nodate nonumber;

data progen;

input prog bloco $\mathrm{p}$;

$\mathrm{t}=\operatorname{arsin}(\operatorname{sqrt}(\mathrm{p}))^{*} 180 / 3.1415927$;

box $=p^{* *} 1.3$;

*dados* $/ *$ Entra com os dados*/

proc mixed method=ML covtest ic;

class prog bloco;

model $\mathrm{p} t$ box $=$ bloco/solution outp=pred;

random $\mathrm{prog} / \mathrm{solution}$;

Ismeans bloco;

run;

data residuo;

set pred;

run;

proc print data=residuo $(\mathrm{keep}=$ prog bloco $\mathrm{p}$ pred resid);

run;

proc capability data=residuo;

var resid;

qqplot;

run;

proc print data $=$ pred (keep=prog bloco p pred resid) noobs;

run;

proc gplot data=residuo;

plot resid*pred;

run; 
ANEXO G - Programa SAS para análise dos dados de progênies de Eucalyptus grandis, considerando o modelo linear generalizado misto com função de ligação logística, pelo método da quase-verossimilhança.

options nodate nonumber;

data progen;

input bloco prog s ns;

cards;

*dados*/*Entra com os dados*/

;

proc print;

run;

data new;

set progen;

do $\mathrm{i}=1$ to $\mathrm{s}$;

$\mathrm{y}=1$;

output;

end;

do $\mathrm{i}=1$ to $\mathrm{ns}$;

$\mathrm{y}=0$;

output;

end;

proc print;

run;

\%include

'C: \Arquivos

de

programas $\backslash S A S$

Institute $\backslash S A S \backslash V 8 \backslash S T A T \backslash S A M P L E \backslash g l i m m i x . s a s ' ;$

$\%$ glimmix (data $=$ new, procopt $=$ method $=$ ml covtest ic, stmts $=\%$ str(class prog bloco;

model $\mathrm{y}=\mathrm{bloco} / \mathrm{s}$

random prog/s; 
Ismeans bloco;

),

error=binomial, link=logit,

out=pred);

run;

proc print data $=$ pred $($ keep $=$ mu resraw $)$;

run; 
ANEXO H - Programa SAS para análise dos dados de progênies de Eucalyptus grandis, considerando o modelo linear generalizado misto com função de ligação logística, pelo método da máxima verossimilhança.

options nodate nonumber;

data progen;

input r n Bloco Prog;

cards;

*dados* $/ *$ Entra com os dados*/

;

proc print;

run;

proc glmmod data $=$ progen outdesign $=$ proge;

class Bloco;

model $\mathrm{r}$ n Prog=Bloco;

run;

proc sort data=proge;

by Prog;

run;

proc nlmixed data $=$ proge qpoints $=16$;

parms Bloco1 Bloco2 Bloco3 Bloco4 Bloco5 Bloco6 Bloco7 Bloco8 Bloco9 Bloco10=0 $\operatorname{sig} 2 \mathrm{p}=1$;

array $x\{10\}$ coll-collo;

array beta $\{10\}$ Bloco1--Blocol0;

eta $=\mathrm{u}$;

do $\mathrm{i}=1$ to 10 ;

eta $=$ eta $+x\{i\} *$ beta $\{i\}$;

end;

$\mathrm{p}=\exp ($ eta $) /(1+\exp ($ eta $))$;

model $r \sim \operatorname{binomial}(n, p)$; 
random $u \sim \operatorname{normal}(0$, sig $2 p)$ subject=Prog out=efeitos;

predict $\mathrm{p}$ out $=\mathrm{p}$;

run;

proc print data $=$ efeitos;

run;

proc print data $=\mathrm{p}$;

run; 


\section{REFERÊNCIAS BIBLIOGRÁFICAS}

ABRAMOWITZ, M.; STEGUN, I. Handbook of mathematical functions. Washington, DC: National Bureau of Standards, 1964. 1046p.

BARBIN, D. Componentes de variância: Teoria e Aplicações. Piracicaba: ESALQ/USP, 1998. 120p.

BORÉM, A. Melhoramento de plantas. 3.ed. Viçosa: UFV, 2001. 500p.

BOX, G.E.P.; COX, D.R. An analysis of transformations. Journal of the Royal Statistical Society, B, v.26, p.211-252, 1964.

BRESLOW, N.E.; CLAYTON, D.G. Aproximate inference in generalized linear mixed models. Journal of the American Statistical Association, v.88, n.421, p.9-25, 1993.

CORDEIRO, G.M. Modelos lineares generalizados. In: SIMPÓSIO NACIONAL DE PROBABILIDADE E ESTATÍSTICA, 7., Campinas, 1986. anais Campinas: Unicamp, 1986. 286p. 
DEMÉTRIO, C.G.B. Modelos lineares generalizados em experimentação agronômica. In: SIMPÓSIO DE ESTATÍSTICA APLICADA À EXPERIMENTAÇÃO AGRONÔMICA, 9.; REUNIÃO ANUAL DA SOCIEDADE INTERNACIONAL DE BIOMETRIA, 46., Piracicaba, 2001. anais Piracicaba: USP/ESALQ, 2001. 113p.

DEMPSTER, A.P.; LAIRD, N.M.; RUBIN, D.B. Maximum likelihood from incomplete data via the EM algorithm. Journal of the Royal Statistical Society. Series B, v. 39, p. $1-38,1977$.

DRAPER, N.R.; SMITH, H. Applied regression analysis. New York: Wiley, 1981. $709 \mathrm{p}$.

EFRON, B.; TIBSHIRANI, R.J. An introduction to the bootstrap. New York: Chapman \& Hall, 1993. 436p.

FALCONER, D. S. Introduction to quantitative genetics. 3. ed. Harlow: Longman, 1989. 438p.

FELLNER, W.R. Robust estimation of variance components. Technometrics, v. 28, p.51-60, 1986.

FELLNER, W.R. Sparse matrix and the estimation of variance components by likelihood methods. Communications in statistics: Theory and Methods, A, v.16, p.439-463, 1987.

FIRTH, D. Generalized linear models. In: HINKLEY, D.V.; REID, N., SNELL, E.J. Statistical Theory and Modelling. London: Chapman \& Hall, 1991 . p.55-82. 
HARTLEY, H.O.; RAO, J.N.K. Maximum-likelihood estimation for the mixed analysis of variance model. Biometrika, v.54, n.1, p. 93-108, 1967.

HARVILLE, D.A. Maximum likelihood approaches to variances components estimation and to relates problems. Journal of the American Statistical Association, v.72, p.320-340, 1977.

HENDERSON, C.R. Aplications of linear models in animal breeding. Ontario: University of Guelph, 1984. 462p.

HENDERSON, C.R. Recent developments in variance and covariance estimation. Journal Animal Science, v.63, p. 208-216, 1986.

HICKS, C.R. Fundamental concepts in the design of experiment. 2.ed. New York: Holt, Rinehart and Winston, 1973. 349p.

JØRGENSEN, B. Exponencial dispersion models (with discussion). Journal of the Royal Statistical Society, Series B, v.49, n.2, p.127-162, 1987.

KNAPP, S.J.; STROUP, W.W.; ROSS, W.M. Exact confidence intervals for heritability on a progeny mean basis. Crop Science, v.25, n.1, p.192-194, 1985.

LAIRD, N.L.; LANGE, N.; STRAM, D. Maximum likelihood computation with repeated measures: application of EM algorithm. Journal of the American Statistical Association, v.82, p.97-105, 1987.

LINDSEY, J.K. Applying generalized linear models. New York: Springer-Verlag, 1997. 256p.

McCULLAGH, P. Quasi-likelihood function. Annals of Statistics, v.11, p.59-67, 1983. 
McCULLAGH, P.; NELDER, J. Generalized Linear Models. 2.ed. London: Chapman and Hall, 1989.511p.

McCULLOCH, C.E. Maximum likelihood variance components estimation for binary data. Journal of the American Statistical Association, v.89, n.425, p.330-335, 1994.

McCULLOCH, C.E.; SEARLE, S.R.. Generalized, Linear, and Mixed Models. New York: John Wiley, 2001. 325p.

MOOD, A.M.; GRAYBILL, F.A.; BOES, D.C. Introduction to the theory of statistics. Tokyo: McGraw-Hill, 1974. 564p.

NELDER, J.A.; WEDDERBURN, R.W.M. Generalized linear models. Journal of the Royal Statistical Society, Series A, v. 135, p.370-384, 1972.

OTSUK, I.P. Influência de transformações de dados sobre a estimação dos componentes de variância. Piracicaba, 1991. 66p. Dissertação (Mestrado) - Escola Superior de Agricultura "Luiz de Queiroz", Universidade de São Paulo.

PATTERSON, H.D.; THOMPSON, R. Recovery of inter-block information when block sizes are unequal. Biometrika, v. 58, p. 545-554, 1971.

PEREIRA NETO, J. Estudo comparativo de métodos de estimação da variância de coeficiente de herdabilidade. Piracicaba, 1994. 80p. Dissertação (Mestrado) - Escola Superior de Agricultura "Luiz de Queiroz", Universidade de São Paulo.

RAMALHO, M.A.P.; SANTOS, J.B dos; PINTO, C.A.B.P. Genética na Agropecuária. Lavras: UFLA, 2000. 472p. 
SAS Institute Inc. Release 8.02, Cary, NC: SAS Institute, 2001.

SEARLE, S.R. Linear models for unbalanced data. New York: John Wiley, 1987. $536 \mathrm{p}$.

SEARLE, S.R.; CASELlA, G.; McCULlOCH, C.E. Variance Components. New York: John Wiley, 1992. 501p.

SCHALL, R. Estimation in generalized linear models with random effects. Biometrika, v.78, p.719-727, 1991.

SNEDECOR, G.W.; COCHRAN, W.G. Statistical Methods. 8. ed. Iowa: Iowa State University Press, 1989. 503p.

STEEL, R.G.D.; TORRIE, J.H. Principles and procedures of statistics. 2.ed. New York: McGraw-Hill, 1980.633p.

STIRATELLI, R.; LAIRD, N.; WARE, J.H. Random-effects models for serial observations with binary response. Biometrics, v.40, p.961-971, 1984.

THOMPSON, R. The problem of negatitive estimates of variance components. Annals of Mathematics Statistics, v. 33, p. 273-275, 1962.

VENCOVSKY, R. Análise de variância de freqüências alélicas. Revista Brasileira de Genética, v.15, n.1, supl, p.53-60, 1992.

WEDDERBURN, R.W.M. Quasi-likelihood functions, generalized linear models and the Gauss-Newton method. Biometrika, v.61, p.439-447, 1974. 
WOLFINGER, R. Laplace's approximation for nonlinear mixed models. Biometrika, v.80, n.4, p.791-795, 1993.

WOLFINGER, R.; O'CONNELL, M. Generalized linear models; a pseudo-likelihood approach. Journal of the Statistical Computation and Simulation, v. 48, p.233$243,1993$. 